\title{
Simple Type and the Boundary of Moduli Space
}

\author{
David Groisser* \\ Department of Mathematics \\ University of Florida \\ Gainesville FL 32611-8105 \\ groisser@math.ufl.edu
}

\author{
Lorenzo Sadun ${ }^{\dagger}$ \\ Department of Mathematics \\ University of Texas \\ Austin, TX 78712 \\ sadun@math.utexas.edu
}

November 14, 2018

\begin{abstract}
We measure, in two distinct ways, the extent to which the boundary region of moduli space contributes to the "simple type" condition of Donaldson theory. Using a geometric representative of $\mu(\mathrm{pt})$, the boundary region of moduli space contributes $6 / 64$ of the homology required for simple type, regardless of the topology or geometry of the underlying 4-manifold. The simple type condition thus reduces to the interior of the $k+1$ st ASD moduli space, intersected with two representatives of ( 4 times) the point class, being homologous to 58 copies of the $k$-th moduli space. This is peculiar, since the only known embeddings of the $k$-th moduli space into the $k+1$ st involve Taubes gluing, and the images of such embeddings lie entirely in the boundary region.

When using de Rham representatives of $\mu(\mathrm{pt})$, the boundary region contributes $1 / 8$ of what is needed for simple type, again regardless of the topology or geometry of the underlying 4-manifold. The difference between this and the geometric representative answer is surprising but not contradictory, as the contribution of a fixed region to the Donaldson invariants is geometric, not topological.
\end{abstract}

1991 AMS Subject Classification: 57R57 (58D27, 53C07, 58G99)

Key Words: simple type, Donaldson theory, $\mu$-map, Yang-Mills

\footnotetext{
*Research supported in part by National Science Foundation grant DMS-9307648

${ }^{\dagger}$ Research supported in part by National Science Foundation grant DMS-9626698, an NSF Mathematical Sciences Postdoctoral Fellowship and Texas ARP Grant 003658-037
} 


\section{Introduction}

This paper is a study in the geometry and topology of anti-self-dual Yang-Mills moduli spaces. Although moduli spaces were studied extensively for their own sake in the 1970s and early 1980s, in the late 1980s and early 1990s such studies were primarily a means to an end. Moduli spaces were studied to compute Donaldson invariants, and Donaldson invariants were computed for their applications to classifying smooth 4-manifolds. Seiberg-Witten theory has, of course, made that last road obsolete. It is believed that the Seiberg-Witten invariants determine the Donaldson invariants, and the former are far easier to handle.

However, Seiberg-Witten theory has opened up new uses for Donaldson theory. From Seiberg-Witten theory, we now have a much better understanding of Donaldson invariants. Instead of using moduli spaces as a tool for computing Donaldson invariants, we can now use Donaldson invariants as a tool for understanding moduli spaces. This paper is an exercise along those lines.

A basic problem in four-dimensional gauge theory is to understand the "simple type" condition. In Donaldson theory, a manifold is said to have simple type if its Donaldson invariants satisfy a certain recursion relation ([KM]; see (1.2) below). In Seiberg-Witten theory, a manifold has simple type if it has no Seiberg-Witten classes of nonzero index. The two notions of simple type are believed to be equivalent, so that theorems proved about one form of simple type should yield information about the other.

In this paper we work with the Donaldson theory sense of simple type, examining what simple type implies about the geometry of anti-self-dual moduli spaces. In two ways - with intersection theory and with de Rham theory - we measure the extent to which the boundary region of moduli space contributes to the simple type recursion relation. Our results imply that the anti-self-dual moduli spaces associated to any manifold of simple type have a very surprising interior geometric structure. Widely satisfied sufficient conditions are known for a manifold to be of simple type [KM], and it is conjectured that indeed all 4-manifolds with $b_{+}>1$ are of simple type. Hence our results apply to a great many manifolds.

Simple type says that the $k+1$ st moduli space $\mathcal{M}_{k+1}$, intersected with certain varieties, has the homology of a certain multiple of the $k$-th moduli space $\mathcal{M}_{k}$. Our intersection theory approach is based on the construction in [S1] of a geometric representative of $\mu$ of a point (see below). Using this representative we show that the portion of (a small perturbation of) $\mathcal{M}_{k+1}$ near the boundary contributes $6 / 64$ of the homology required for simple type, regardless of the topology or geometry of the underlying 4-manifold. (For a quick, heuristic derivation of this 6/64, see [S2].) Simple type thus reduces to a statement relating $\mathcal{M}_{k}$ to nontrivial structure in the interior of $\mathcal{M}_{k+1}$ (unless our small perturbation of $\mathcal{M}_{k+1}$ is drastically unfaithful topologi- 
cally, which seems highly unlikely). This is surprising, since the only known relations between $\mathcal{M}_{k}$ and $\mathcal{M}_{k+1}$ involve Taubes patching, and relate $\mathcal{M}_{k}$ to the boundary of $\mathcal{M}_{k+1}$.

Our second approach is to use differential form representatives of the images of the $\mu$ map. One then takes the wedge product of these forms and integrates over $\mathcal{M}_{k+1}$. If we restrict the domain of integration to a neighborhood of the boundary of $\mathcal{M}_{k+1}$, we can reinterpret the simple type condition in terms of the integral of a certain 8-form over a submanifold that represents the space of "bubble parameters" in the neighborhood of a background connection in $\mathcal{M}_{k}$. We show that, again independently of the topology and geometry of the base manifold, this integral has precisely $1 / 8$ the value of what one would naively expect if simple type were captured purely by a neighborhood of the boundary. Thus again simple type becomes a statement about the nontrivial structure of the interior of moduli space.

It is curious but no contradiction that the two approaches yield the different numerical answers 6/64 and 1/8. While the Donaldson polynomial is topological, hence independent of the choice of geometric or de Rham representatives, the contribution of each region of moduli space is geometric, and need not be the same for two different representatives. Indeed, the de Rham and geometric representative calculations not only disagree on the contribution of the boundary region, but also disagree on how close to the boundary the essential contributions are. In terms of the small parameter $L$, described below, the geometric representative picks up contributions from bubbles of size $O\left(L^{2}\right)$, while the bulk of the support of the de Rham representative is on bubbles of size $O(L)$.

To state our results more precisely, we must review the definition of the Donaldson invariants, and of simple type. Let $N$ be an oriented 4-manifold, let $G=S U(2)$ or $S O(3)$, and let $\mathcal{B}_{k}^{*}$ be the space of irreducible connections (up to gauge equivalence) on $P_{k}$, the principal $G$ bundle of instanton number $k$ over $N$. Let $\mathcal{M}_{k} \subset \mathcal{B}_{k}$ be the space of irreducible connections on $P_{k}$ with anti-self-dual curvature, modulo gauge transformations. We will frequently omit the index $k$.

Donaldson [D1, D2] defined a map $\mu: H_{i}(N, \mathbf{Q}) \rightarrow H^{4-i}\left(\mathcal{B}_{k}^{*}, \mathbf{Q}\right), i=0,1,2,3$, whose image freely generates the rational cohomology of $\mathcal{B}_{k}^{*}$. Donaldson invariants are then defined by pairing the fundamental class of $\mathcal{M}_{k}$ with products of $\mu$ of the homology classes of $N$, where $k$ is chosen so that the dimensions match. Formally, for elements $\left[\Sigma_{1}\right], \ldots,\left[\Sigma_{n}\right] \in H_{*}(N)$, we write

$$
D\left(\left[\Sigma_{1}\right] \ldots\left[\Sigma_{n}\right]\right)=\mu\left(\left[\Sigma_{1}\right]\right) \smile \cdots \smile \mu\left(\left[\Sigma_{n}\right]\right)\left[\mathcal{M}_{k}\right] .
$$

Now let $x$ be the point class in $H_{0}(N)$, and let $\omega$ be any formal product of classes in $H_{*}(N)$. The simple type condition is that, for all $\omega$,

$$
D\left(x^{2} \omega\right)=4 D(\omega)
$$


Of course, the "fundamental class of $\mathcal{M}_{k}$ " is usually not well defined, as $\mathcal{M}_{k}$ is typically not compact. The usual way to make sense of (1.1) and (1.2) is with geometric representatives. One finds finite-codimension varieties $V_{\Sigma}$ in $\mathcal{B}_{k}^{*}$ that are Poincaré dual to $\mu([\Sigma])$. One then counts points, with sign, in $V_{\Sigma_{1}} \cap \cdots \cap V_{\Sigma_{n}} \cap \mathcal{M}_{k}$. To make a topological invariant one must show that the number of intersection points is independent of auxiliary data, such as the metric and the choice of representatives. This requires careful analysis of the bubbling-off phenomena that make $\mathcal{M}_{k}$ noncompact.

To compute the left-hand side of (1.2) we need a variety that represents $\mu$ of the point class $x$. In general $\mu(x)$ is not an integral class in $H^{4}\left(\mathcal{B}^{*}\right)$ and strictly speaking has no geometric representative. However, $-4 \mu(x)$ is an integral class. For $p \in N$, let

$$
\nu_{p}=\left\{[A] \in \mathcal{B}_{k+1}^{*} \mid F_{A}^{-} \text {is reducible at } p\right\} .
$$

Here $F_{A}^{-}=\left(F_{A}-* F_{A}\right) / 2$ is the anti-self-dual part of the curvature $F_{A}$, and by "reducible at $p$ " we mean that the components $F_{i j}^{-}(p)$ are all colinear as elements of the Lie algebra of $G$. In [S1] it was shown that $\nu_{p}$ is a geometric representative of $-4 \mu([p])$. The simple type condition can therefore be rewritten as

$$
\#\left(\mathcal{M}_{k+1} \cap \nu_{p} \cap \nu_{q} \cap V_{\omega}\right)=64 \#\left(\mathcal{M}_{k} \cap V_{\omega}\right),
$$

where $p$ and $q$ are any two points in $N, \omega$ is an arbitrary formal product of homology cycles of $N$, and $V_{\omega}$ is a geometric representative of $\mu(\omega)$.

More formally, one can write

$$
\left[\mathcal{M}_{k+1} \cap \nu_{p} \cap \nu_{q}\right]=64\left[\mathcal{M}_{k}\right]
$$

Strictly speaking, the left hand side is an element of $H_{*}\left(\mathcal{B}_{k+1}\right)$, while the right hand side is in $H_{*}\left(\mathcal{B}_{k}\right)$. However, $\mathcal{B}_{k}$ and $\mathcal{B}_{k+1}$ are homotopy equivalent spaces, and their homology classes may be identified.

The first question studied in this paper is this: Suppose $p$ and $q$ are extremely close points in $N$, separated by a distance $2 L$. How many of the points on the left hand side of (1.4) lie near the boundary of $\mathcal{M}_{k+1}$ ? The answer is quite simple:

Theorem 1.1 Let $N$ be a compact oriented Riemannian four-manifold of arbitrary topology and geometry and let $4 k \geq 3 b_{+}+5$. Fix a coordinate patch on $N$, and let $p$ and $q$ be the points $( \pm L, 0,0,0)$. Fix $\omega \in \operatorname{Sym}^{*}\left(H_{*} N\right), K>0$, and $\alpha \in(0,2)$. Let $\tilde{\mathcal{M}}_{k+1}^{0}$ be the portion of the (perturbed) moduli space $\tilde{\mathcal{M}}_{k+1}$ consisting of a background of charge $k$ and a charge-one bubble of size $\lambda<K L^{\alpha}$. For generic choices of geometric representatives $V_{\omega}$ of $\mu(\omega)$, and for all sufficiently small $L$, the intersection number of $\tilde{\mathcal{M}}_{k+1}^{0}$ with $V_{\omega} \cap \nu_{p} \cap \nu_{q}$ is $6 D(\omega)$.

The perturbed moduli space $\tilde{\mathcal{M}}_{k+1}$ is constructed, and the genericity conditions specified, in $\S \S 3-4$. This theorem is restated, more precisely, as Theorem 4.1. In this 
theorem, and throughout $\S \S 2-4$, we assume $k$ is in the indicated "stable range" to avoid contributions from lower strata of the compactified moduli space.

Simple type thus reduces to a statement that the interior of $\mathcal{M}_{k+1} \cap \nu_{p} \cap \nu_{q}$ be homologous to 58 copies of $\mathcal{M}_{k}$. This is striking, since in general very little is known about the interior of $\mathcal{M}_{k+1}$. As noted earlier, the only known embeddings of $\mathcal{M}_{k}$ into $\mathcal{M}_{k+1}$ involve Taubes patching, and have an image near the boundary of $\mathcal{M}_{k+1}$.

On the level of differential forms, the de Rham-theoretic version of the $\mu$-map is represented by a map

$$
\mu_{d}: \Omega^{i}(N) \rightarrow \Omega^{i}\left(\mathcal{B}_{k+1}^{*}\right), \quad i=0, \ldots, 4
$$

the argument of $\mu_{d}$ is a form representing the Poincaré dual of the argument of $\mu$. In particular, $\mu(x)$ is represented by a 4 -form $\mu_{d}(\omega) \in H^{4}\left(\mathcal{B}_{k+1}^{*}\right)$ for any $\omega \in \Omega^{4}(N)$ with $\int_{M} \omega=1$.

One can write down an explicit formula for such a representative $\mu_{d}(\omega)$ by appealing to Chern-Weil theory on the canonical $S O(3)$-bundle $\mathcal{P} \rightarrow \mathcal{B}_{k+1}^{*} \times M$ (see $\S 5$ ). Furthermore given $p \in M$, if we replace $\omega$ by $\delta_{p}$, a delta-form supported at a point $p$, then the resulting form on $\mathcal{B}_{k+1}^{*}$ is still de Rham cohomologous to a form obtained using smooth $\omega$ (although there is an important difference that we will discuss later). Let us write $\mu_{d}(p):=\mu_{d}\left(\delta_{p}\right)$. Note that for smooth $\omega \in \Omega^{4}(N)$ we have

$$
\left.\mu_{d}(\omega)\right|_{A}=\left.\int_{N} \mu_{d}(p)\right|_{A} \omega(p)
$$

If $Z$ is an eight-dimensional cycle in $\mathcal{B}_{k+1}^{*}$, then since the cohomology class of $\mu_{d}(p)$ is independent of $p$, for any points $p, q \in M$ we have $\int_{Z} \mu_{d}(p) \wedge \mu_{d}(p)=\int_{Z} \mu_{d}(p) \wedge \mu_{d}(q)$, and moreover this integral depends only on the homology class of $Z$.

Pretend, for a moment, that the moduli spaces $\mathcal{M}_{k+1}, \mathcal{M}_{k}$ are the total space and base space of a compact, connected, oriented fiber bundle $\pi: \mathcal{M}_{k+1} \rightarrow \mathcal{M}_{k}$; the fibers would then be mutually cohomologous compact submanifolds $Z \subset \mathcal{M}_{k+1}$. For any form $\phi \in \Omega^{\text {top }}\left(\mathcal{M}_{k}\right)$, we would have a product formula

$$
\int_{\mathcal{M}_{k+1}} \mu_{d}(p) \wedge \mu_{d}(q) \wedge \pi^{*} \phi=\left(\int_{Z} \mu_{d}(p) \wedge \mu_{d}(q)\right)\left(\int_{\mathcal{M}_{k}} \phi\right)
$$

(assuming compatible orientations), so the simple-type condition (1.2) would be equivalent to

$$
\int_{Z} \mu_{d}(p) \wedge \mu_{d}(q)=4
$$

In reality the moduli spaces are not compact and there is no such global fibration. However, from the current understanding of Donaldson invariants one might speculate that the relevant integrals are supported in a region near the ideal boundary of $\mathcal{M}_{k+1}$. 
Of course a random de Rham representative of a cohomology class can be supported wherever it likes, but $\mu_{d}(\cdot)$ is not random, and there is evidence that its properties near the boundary of moduli space do indeed capture a lot of cohomological information. For example, consider the five-dimensional moduli spaces of 1-instantons over simply connected manifolds with $b_{+}=0$. In such cases the inverse of a collar map gives embeddings $\tau_{\lambda}: N \rightarrow \mathcal{M}_{1} \subset \mathcal{B}^{*}$ for $\lambda$ sufficiently small (the image of $\tau_{\lambda}$ consisting of instantons of scale $\lambda$ ), and one has Donaldson's theorem that the composition $\tau_{\lambda}^{*} \circ \mu: H_{2}(N, \mathbf{Z}) \rightarrow H^{2}(N, \mathbf{Z})$ is precisely Poincaré duality ([DK Corollary 5.3.3]). The corresponding assertion in de Rham cohomology would be that $\tau_{\lambda}^{*} \circ \mu_{d}: \Omega^{2}(N) \rightarrow$ $\Omega^{2}(N)$ induces the identity on cohomology. But in fact in this context one can show that $\lim _{\lambda \rightarrow 0} \tau_{\lambda}^{*} \circ \mu_{d}$ is already the identity map on the level of forms, in all degrees ([GP3]). This fact extends to more general moduli spaces $\mathcal{M}_{k}$ near the "maximal bubbling" ends consisting of instantons with $k$ distinct charge-one bubbles.

If we consider that portion $\mathcal{M}_{k+1, \lambda_{0}}^{\prime}$ of $\mathcal{M}_{k+1, \lambda_{0}}$ near the highest-dimensional boundary stratum $\mathcal{M}_{k} \times N$, there is indeed a fibration $\mathcal{M}_{k+1, \lambda_{0}}^{\prime} \rightarrow \mathcal{M}_{k}^{\prime}$ whose fibers can be identified with subsets of an 8-dimensional space of framed ASD connections on $\mathbf{R}^{4}$. (Here $\mathcal{M}_{k}^{\prime}$ denotes the space of non-concentrated irreducible $k$-instantons, and $\mathcal{M}_{k+1, \lambda_{0}}^{\prime}$ the space of $(k+1)$-instantons with only a single "bubble", of charge one, and scale less than some small number $\lambda_{0}$.) The typical fiber $Z=Z_{\lambda_{0}}$ is itself a bundle over $\left(0, \lambda_{0}\right) \times N$ for some small $\lambda_{0}$, whose fiber over $(\lambda, p) \in\left(0, \lambda_{0}\right) \times N$ is the space of "gluing parameters" $\operatorname{Hom}_{S O(3)}\left(\Lambda_{+}^{2} T^{*} N, A d P_{k}\right) \cong S O(3)$ (see [DK, p. 324]). Since $\mathcal{M}_{k+1, \lambda_{0}}^{\prime}$ is such a large portion of the end of $\mathcal{M}_{k+1}$, one might then expect that an approximate version of (1.9) holds under the assumption of simple type.

What we show below is that (1.9) fails in a very precise way: independent of the topology and geometry of $N$, if $\lambda_{0}$ is small enough and $\operatorname{dist}(p, q)$ is small compared to $\lambda_{0}$ (but nonzero), then

$$
\int_{Z_{\lambda_{0}}} \mu_{d}(p) \wedge \mu_{d}(q) \approx \frac{1}{2}
$$

under certain technical but intuitively reasonable assumptions about the fiber $Z_{\lambda_{0}}$. Taking a limit as $q \rightarrow p$ and then as $\lambda_{0} \rightarrow 0$, the integral above approaches an integral over the space of framed instantons on $\mathbf{R}^{4}$, and this latter integral has the precise value $1 / 2$.

At this stage the reader may wonder why we do not simply take $p=q$ in (1.10). The reason is that for purposes of integration, the $\mu_{d}(p)$ turn out to be more singular than the representatives $\mu_{d}(\omega)$ for smooth $\omega$. Were we to set $p=q$ in $(1.10), 1 / 2$ would be replaced by 0 . This discontinuity can be modeled by the following twodimensional example. Let $H$ be the upper half-plane $\left\{(x, \lambda) \in \mathbf{R}^{2} \mid \lambda>0\right\}$ and for each $L \in \mathbf{R}$ let $\theta_{L}: H \rightarrow(0, \pi)$ be the usual polar-coordinate angle as measured from $(L, 0)$ (so $\left.d \theta_{L}=((x-L) d \lambda-\lambda d x) /\left((x-L)^{2}+\lambda^{2}\right)\right)$. As forms on $H$, the $d \theta_{L}$ are all cohomologous (in fact cohomologous to zero). However, $\int_{H} d \theta_{0} \wedge d \theta_{0}=0$, while for $L>0$ we have we have $\int_{H} d \theta_{0} \wedge d \theta_{L}=\pi^{2} / 2$. Essentially, $\mu_{d}(p) \wedge \mu_{d}(q)$ behaves like a 
quaternionic version of this example.

The technical assumptions on the fiber $Z$ are enumerated as $(\mathbf{Z 1}-\mathbf{Z 5})$ in section 7 . The first three of these assumptions are known to be satisfied by the fiber constructed in $[\mathrm{DK}]$, but we have not determined whether the latter two are satisfied. These two are assumptions on the tangent space to $T_{[A]} Z$, where $[A] \in Z$, and we prove that they are satisfied by a subspace of $T_{[A]} \mathcal{M}$ (the "approximate tangent space") that we argue is close to $T_{[A]} Z$. Because this step is only a plausibility argument, (1.10) implies one of two things: either $\mu_{d}(p) \wedge \mu_{d}(q)$ has most of its support in the interior of moduli space (or near higher codimension boundary strata), or the intuitive picture of the fiber $Z$ is significantly wrong. Either way, the conclusion is surprising.

Our second main theorem is then

Theorem 1.2 Let $N$ be a compact oriented Riemannian four-manifold of arbitrary topology and geometry and let $k \geq 1$. Assume that a typical fiber $Z_{\lambda_{0}}$ of the fibration $\mathcal{M}_{k+1, \lambda_{0}}^{\prime} \rightarrow \mathcal{M}_{k}^{\prime}$ satisfies $(\mathbf{Z 1}-\mathbf{Z 5})$ of $\S 7$. Then for any $p, q \in N$, the form $\mu_{d}(p) \wedge \mu_{d}(q)$ is integrable over $Z_{\lambda_{0}}$ for $\lambda_{0}$ sufficiently small, and

$$
\lim _{\lambda_{0} \rightarrow 0}\left(\lim _{q \rightarrow p} \int_{Z_{\lambda_{0}}} \mu_{d}(p) \wedge \mu_{d}(q)\right)=\frac{1}{2},
$$

while

$$
\lim _{\lambda_{0} \rightarrow 0} \int_{Z_{\lambda_{0}}} \mu_{d}(p) \wedge \mu_{d}(p)=0 .
$$

The convergence in (1.11)-(1.12) is uniform in $p, q$. Hence if $\tilde{\delta}_{p, L}$ denotes a smooth 4-form on $N$ of total integral 1, supported in a ball of radius $L$ about $p$, then (using (1.7))

$$
\lim _{\lambda_{0} \rightarrow 0}\left(\lim _{L \rightarrow 0} \int_{Z_{\lambda_{0}}} \mu_{d}\left(\tilde{\delta}_{p, L}\right) \wedge \mu_{d}\left(\tilde{\delta}_{p, L}\right)\right)=\frac{1}{2} .
$$

By uniform convergence in (1.11) we mean that for all $\epsilon>0$ there exist $\lambda_{1}, \delta(\cdot)>0$ such if $0<\lambda_{0}<\lambda_{1}$ and $0<\operatorname{dist}(p, q)<\delta\left(\lambda_{0}\right)$ then the integral in (1.11) differs from $1 / 2$ by less than $\epsilon$.

It is not necessary to take the limits in (1.11) completely independently, as long as $q \rightarrow p$ much faster than $\lambda_{0} \rightarrow 0$. If, for example, we require that $\operatorname{dist}(p, q)=$ const $\lambda_{0}^{1+\alpha}$ for some $\alpha>0$, and then take a limit as $\lambda_{0} \rightarrow 0$, we again get $1 / 2$.

Note that if we held $p$ and $q$ fixed rather than taking $\lim _{q \rightarrow p}$ in (1.11), the limit as $\lambda_{0} \rightarrow 0$ would necessarily be zero ( since $\mu_{d}(p) \wedge \mu_{d}(q)$ is integrable). It turns out that for $q \neq p$ the integrand in (1.11) is supported in a region in which $\lambda$ is of order $\operatorname{dist}(p, q)$. Thus if we wish to extend $\mu_{d}(p)$ and $\mu_{d}\left(\tilde{\delta}_{p, L}\right)$ to forms on the Uhlenbeck compactification of $\mathcal{M}$, with $\lim _{L \rightarrow 0} \mu_{d}\left(\tilde{\delta}_{p, L}\right)=\mu_{d}(p)$ in a distributional sense, then 
$\mu_{d}(p) \wedge \mu_{d}(p)$ should be viewed as the sum of a delta-form supported on the boundary of moduli space and a smooth form supported away from the boundary.

Note also that Theorem 1.2 does not require $k$ to be in the "stable range", unlike Theorem 1.1. However, Theorem 1.2 is most interesting for $k$ in the stable range, since only then can the Donaldson invariant $D\left(\left[\Sigma_{1}\right] \cdots\left[\Sigma_{n}\right]\right)$ be expressed as a topologically invariant integral $\int_{\mathcal{M}} \mu_{d}\left(\omega_{1}\right) \wedge \cdots \wedge \mu_{d}\left(\omega_{n}\right)$.

The rest of this paper is organized into two main parts, with $\S \S 2-4$ devoted to proving Theorem 1.1 and $\$ \S 5-10$ devoted to proving Theorem 1.2. The strategy of proof, and the division of the paper, is as follows:

Let $A$ be a connection obtained by gluing a small bubble onto a background connection $A_{0}$. It turns out that the curvature of $A$ is well approximated by the sum of the curvature $F_{0}$ of $A_{0}$ and the curvature $F_{\text {std }}$ of a standard $k=1$ instanton, viewed in the correct gauge. We are thus led to the following model problem: Given a connection $\left[A_{0}\right] \in \mathcal{M}_{k}$ and two closely spaced points $p$ and $q$, for how many triples $(x, \lambda, g)$ is the sum of the curvature $F_{0}$ of $A_{0}$ and the curvature $F_{\text {std }}$ of a standard instanton, centered at $x$ with size $\lambda$ and gluing angle $g$, reducible at both $p$ and $q$ ? In $\S 2$ we solve this model problem and show that, for generic $A_{0}$, the answer is 6 .

In $\S 3$ we construct a family of approximately ASD connections, based on an explicit gluing formula. This is a perturbation, which we denote $\tilde{\mathcal{M}}_{k+1}$, of the boundary region of $\mathcal{M}_{k+1}$. We check explicitly that in this family the curvature is well approximated by $F_{0}+F_{\text {std }}$. By linearly interpolating between $F_{0}+F_{\text {std }}$ and the actual curvatures of connections in $\tilde{\mathcal{M}}_{k+1}$, we show that corresponding to each generic $A_{0} \in \mathcal{M}_{k}$ there are exactly 6 points in $\tilde{\mathcal{M}}_{k+1} \cap \nu_{p} \cap \nu_{q}$ with $\lambda$ sufficiently small.

In $\S 4$ we apply these results to show that if we consider only the boundary region of the (perturbed) moduli space, we obtain (1.5) with 6 on the right-hand side rather than 64 , thereby completing the proof of Theorem 1.1 .

Ideally, one would then like to interpolate from $\tilde{\mathcal{M}}_{k+1}$ to $\mathcal{M}_{k+1}$. This is quite difficult, as $\nu_{p}$ and $\nu_{q}$ are defined by pointwise conditions on the curvature. We know of no pointwise estimates relating the curvature of an almost-ASD connection to that of a nearby ASD connection. In order to make use of the integral estimates available in the literature one would have to replace $\nu_{p}$ and $\nu_{q}$ by geometric representatives defined by integral conditions. While certainly possible, this is beyond the scope of this paper.

We prove Theorem 1.2 by exhibiting $\mu_{d}(p)$ as a purely local piece $\mu_{\text {loc }}(p)$ plus a nonlocal remainder. The local piece dominates in (1.11): as $q \rightarrow p$ the integral of $\mu_{\text {loc }}(p) \wedge \mu_{\text {loc }}(q)$ approaches a calculable integral on $\mathbf{R}^{8}$, with value $1 / 2$, independent of $\lambda_{0}$. (However, $\mu_{\mathrm{loc}}(p) \wedge \mu_{\mathrm{loc}}(p) \equiv 0$.) We establish (1.11)-(1.12) by showing that the integral of the remainder terms in $\mu(p) \wedge \mu(q)$ approaches zero as $\lambda_{0} \rightarrow 0$, independent of $p$ and $q$. Thus taking a limit as $q \rightarrow p$ is relevant only to the purely local part 
of $\mu_{d}(p) \wedge \mu_{d}(q)$ (and taking a limit as $\lambda_{0} \rightarrow 0$ is relevant only to the nonlocal part); the delta-form behavior of $\mu_{d}(p) \wedge \mu_{d}(p)$ is due solely to $\mu_{\text {loc }}(p) \wedge \mu_{\text {loc }}(p)$. The uniformity assertion in Theorem 1.2 follows from the proofs of (1.11)-(1.12), and the final assertion (1.13) then follows from (1.7).

In $\S 5$ we begin our work on Theorem 1.2 by constructing the de Rham representatives $\mu_{d}(p)$. The splitup $\mu_{d}(p)=\mu_{\text {loc }}(p)+$ remainder is based on the "approximate tangent space" mentioned above. This approximation is built by lifting the action of certain vector fields on $N$ to $\mathcal{B}^{*}$. In $\S 6$ we discuss this lifted action (the "canonical flow"), use it to define the approximate tangent spaces $\mathcal{H}_{A}$, and discuss how close the $\mathcal{H}_{A}$ are to being tangent to $\mathcal{M}$. We then exhibit the relation between a fiber constructed from the canonical flow (whose tangent space is essentially the projection to $T_{[A]} \mathcal{M}$ of approximate tangent space above) and the fiber constructed in [DK]. This digression is needed to motivate the technical assumptions (Z1-Z5) given and discussed in $\S 7$. In $\S 8$ we return to the main track, defining $\mu_{\text {loc }}(p)$ and computing the limiting integral of $\mu_{\mathrm{loc}}(p) \wedge \mu_{\mathrm{loc}}(q)$. $\S \S 9-10$ are devoted to a study of the remainder terms $\mu_{d}(p) \wedge \mu_{d}(q)-\mu_{\mathrm{loc}}(p) \wedge \mu_{\mathrm{loc}}(q)$. In $\S 9$ we state the main technical theorem that yields the pointwise norm of these terms (Proposition 9.2), and use this theorem to establish that the integral of the remainder terms tends to zero as $\lambda_{0} \rightarrow 0$. Finally, in $\S 10$, we prove Proposition 9.2. It is this section that contains the core of the analysis underpinning the validity of all the earlier calculations. The estimates in $\S 10$ require a weighted Sobolev inequality, proven in the appendix, that the authors have not seen elsewhere.

The authors thank the 1994 Park City Mathematics Institute, where this work was begun, the National Science Foundation, and the Texas Advanced Research Program. We also thank Dan Freed, Tom Parker, Cliff Taubes, and Karen Uhlenbeck for helpful insights and criticism, and Margaret Combs for assistance with the figures.

\section{The Model Intersection Theory Calculation}

In this section we begin to compare the boundary region of $\mathcal{M}_{k+1} \cap \nu_{p} \cap \nu_{q}$ with $\mathcal{M}_{k}$, by looking at a model problem. Pick a small neighborhood $\tilde{U}$ of our manifold $N$ and give it a flat metric with corresponding Euclidean coordinates. Let $U$ be the corresponding ball in $\mathbf{R}^{4}$. We will denote points either by four real coordinates $\left(x^{0}, \ldots, x^{3}\right)$ or by a single quaternionic coordinate $x^{0}+i x^{1}+j x^{2}+k x^{3}$. Let $p$ and $q$ be the points $( \pm L, 0,0,0)$. Let $A_{0}$ be an ASD connection on $N$, expressed in a smooth gauge on $\tilde{U}$.

An important notational tool is the identification of ASD curvatures with $3 \times 3$ real matrices. Let $F_{0}$ be the pullback, to $U$, of the curvature $F_{A_{0}}$ of an ASD connection on $\tilde{U}$. Relative to the standard oriented basis of $\Lambda_{-}^{2} T^{*} \mathbf{R}^{4}\left(\omega_{1}=d x^{0} d x^{1}-d x^{2} d x^{3}\right.$, 
$\left.\omega_{2}=d x^{0} d x^{2}-d x^{3} d x^{1}, \omega_{3}=d x^{0} d x^{3}-d x^{1} d x^{2}\right), F_{0}$ has at each point 3 Lie-algebravalued components, and so can be viewed as a triple of 3 -vectors. We package this triple of vectors into a $3 \times 3$ real matrix, which we denote $\operatorname{Mat}\left(F_{0}\right)$. More precisely, the first, second and third columns of $\operatorname{Mat}\left(F_{0}\right)$ are half the $\omega_{1}, \omega_{2}$ and $\omega_{3}$ components of $F_{0}$, while the first, second and third entries of each column refer to the three directions in $\mathfrak{s u}(2)$, the Lie Algebra of $S U(2) . A_{0}$ is reducible at a point if and only if $\operatorname{Mat}\left(F_{0}\right)$ has rank 1 (or 0 ) there.

Of course, this construction is dependent on gauge and a choice of basis for $T N$. A gauge transformation is a change of basis in $\mathfrak{s u}(2)$, and thus changes $\operatorname{Mat}\left(F_{0}\right)$ by left-multiplication by an element of $S O(3)$. A change of basis in $T N$ changes $\operatorname{Mat}\left(F_{0}\right)$ by right-multiplication by an element of $S O(3)$. Thus the singular values of $\operatorname{Mat}\left(F_{0}\right)$, and in particular the rank of $\operatorname{Mat}\left(F_{0}\right)$, are gauge- and basis-independent. We shall frequently be thinking of curvatures as $3 \times 3$ matrices in this way. When the context is clear, we will omit the explicit function "Mat".

Now consider a standard $k=1$ instanton on $\mathbf{R}^{4}$ of scale size $\lambda$ and center $y$, viewed in a radial gauge that is singular at $y$ and regular at $\infty$. There are many such gauges, parametrized by a gluing angle $m \in S O(3)$. For fixed $(y, \lambda, m)$, let $F_{\text {std }}$ be the curvature of this connection restricted to $U$.

Let $A$ be an ASD connection obtained by gluing in a bubble with data $(y, \lambda, m)$ to the background $A_{0}$. In $\S 3$ we shall see that $F_{A}$, in an appropriate gauge, is approximately equal to $F_{0}+F_{\text {std }}$. This reduces our main question to the following model problem:

When $L$ is small, for what values of $(y, \lambda, m)$, with $\lambda$ small, is $F_{0}+F_{\text {std }}$ reducible at both $p$ and $q$ ?

Of course, to obtain sensible answers, we must define what we mean by $\lambda$ being "small". Pick constants $K>0$ and $\alpha \in(0,2)$. We say $\lambda$ is small (or that the corresponding bubble is small) if $\lambda<K L^{\alpha}$. The set of gluing data for small bubbles near $p$ and $q$ is $B=U \times\left(0, K L^{\alpha}\right) \times S O(3)$. Let $\tilde{\nu}_{p}$ (resp. $\tilde{\nu}_{q}$ ) be the set of points $(\lambda, y, m) \in B$ such that $F_{0}(p)+F_{\text {std }}(p)$ (resp. $\left.F_{0}(q)+F_{\text {std }}(q)\right)$ is reducible. We must count the intersection points of $\tilde{\nu}_{p}$ and $\tilde{\nu}_{q}$.

Recall that the singular values $\sigma_{1} \geq \sigma_{2} \geq \sigma_{3} \geq 0$ of a $3 \times 3$ real matrix $M$ are the square roots of the eigenvalues of $M^{T} M$. For $M$ generic, these are distinct and positive. The non-generic cases are as follows: Matrices in a codimension-1 set have $\sigma_{3}=0$. Matrices in a codimension-2 set either have $\sigma_{1}=\sigma_{2}$ or $\sigma_{2}=\sigma_{3}$. Matrices in a codimension- 4 set have $\sigma_{2}=\sigma_{3}=0$; these matrices have rank 1 or 0 . Matrices in a codimension- 5 set have $\sigma_{1}=\sigma_{2}=\sigma_{3}$; these are all scalar multiples of $S O(3)$ matrices. Only the zero matrix (codimension-9) has $\sigma_{1}=\sigma_{2}=\sigma_{3}=0$.

Theorem 2.1 Fix $K>0, \alpha \in(0,2)$, and a background connection $A_{0}$. If the singular values of $\operatorname{Mat}\left(F_{0}(0)\right)$ are all distinct, then, for all sufficiently small $L, \tilde{\nu}_{p}$ 
and $\tilde{\nu}_{q}$ intersect at exactly six points. These six intersections are all transverse, and the local intersection number is +1 at each point.

Remark: We shall see that, under the assumptions of the theorem, the intersection points all have $\lambda=O\left(L^{2}\right)$. However, when two of the singular values of $\operatorname{Mat}\left(F_{0}(0)\right)$ are the same, then there are only four intersection points with $\lambda=O\left(L^{2}\right)$. In that case there are generically four additional intersection points with $\lambda=O(L)$. The intersection number of $\tilde{\nu}_{p}$ and $\tilde{\nu}_{q}$ is then 4 if $\alpha>1$ and 8 if $\alpha<1$.

Before beginning the proof of Theorem 2.1 we need some basic facts about $k=$ 1 instantons on $\mathbf{R}^{4}=\mathbf{H}$, we need to fix some conventions, and we need a linear algebra lemma. Think of $S U(2)$ as the unit quaternions, with $\mathfrak{s u}(2)$ as the imaginary quaternions. The connection form of a standard instanton of scale size 1, centered at the origin, is $A_{\text {std }_{0}}=\operatorname{Im}\left(\bar{x} d x /\left(1+|x|^{2}\right)\right)$. The curvature of this connection is

$$
F_{\mathrm{std}_{0}}=\frac{d \bar{x} d x}{\left(1+|x|^{2}\right)^{2}}=\frac{2 i \omega_{1}+2 j \omega_{2}+2 k \omega_{3}}{\left(1+|x|^{2}\right)^{2}}
$$

Note that the matrix $\operatorname{Mat}\left(F_{\mathrm{std}_{0}}\right)$ is $1 /\left(1+|x|^{2}\right)^{2}$ times the identity matrix.

That is in the usual regular gauge, in which $A \sim \phi^{-1} d \phi$ as $|x| \rightarrow \infty$, where $\phi(x)=x /|x|$. We do a gauge transformation by $\phi^{-1}$, to get a radial gauge in which $A=O\left(|x|^{-3}\right.$ ) as $|x| \rightarrow \infty$ (and in which $A$ is singular at the origin). We then do a further gauge transformation by a constant $g_{0}$ to get the most general radial gauge with this property. Let $F_{\text {std }}$ be the curvature form in this gauge. We have $F_{\text {std }}=g_{0}^{-1} \phi F_{\text {std }_{0}} \phi^{-1} g_{0}$. In terms of matrices, $\operatorname{Mat}\left(F_{\text {std }}\right)=\rho\left(g_{0}^{-1}\right) \rho(\phi) \operatorname{Mat}\left(F_{\text {std }_{0}}\right)$, where $\rho$ is the standard double covering map from $S U(2)$ to $S O(3)$; the three columns of $\rho(\phi)$ are $\phi i \phi^{-1}, \phi j \phi^{-1}$, and $\phi k \phi^{-1}$. The matrix $\rho\left(g_{0}\right)$ is our gluing angle $m$.

Now suppose that we have a $k=1$ instanton, centered at a point $y$, with scale size $\lambda$. The curvature matrix, expressed in the exterior radial gauge of gluing angle $m$, is

$$
\operatorname{Mat}\left(F_{\text {std }}(x)\right)=\frac{\lambda^{2}}{\left(\lambda^{2}+|x-y|^{2}\right)^{2}} m^{-1} \rho\left(\frac{x-y}{|x-y|}\right) .
$$

Note that the matrix $\operatorname{Mat}\left(F_{\text {std }}(x)\right)$ is a positive multiple of an $S O(3)$ matrix. The multiple is determined by $\lambda$ and $|x-y|$, while the $S O(3)$ matrix is determined by $m$ and $(x-y) /|x-y|$. (We hencefore will not explicitly distinguish between a curvature and its matrix.)

Our problem is thus one of adding positive multiples of $S O(3)$ matrices to $F_{0}(p)$ and $F_{0}(q)$ to make them reducible. The following lemma is essential.

Lemma 2.2 Let $P$ be a 3 by 3 real matrix with singular values $\sigma_{1} \geq \sigma_{2} \geq \sigma_{3} \geq 0$. If these singular values are all distinct, then there are exactly two pairs $(s, M) \in(0, \infty) \times$ $S O(3)$ for which $P+s M$ has rank 1 (and no pairs $(s, M)$ for which $P+s M=0$ ). 
In both cases $s=\sigma_{2}(P)$. If exactly two of the singular values of $P$ are the same and nonzero, then the two solutions $(s, M)$ coalesce to a double root.

Proof: Let $W=-(P+s M)$. Adding $s M$ to $P$ to make it reducible is the same as decomposing $-P$ as $s M+W$, with $W$ reducible. We therefore count the ways to decompose a matrix $-P$ into the sum of a positive multiple of an $S O(3)$ matrix and a rank 1 matrix. First we show that the desired decompositions can occur only with $s=\sigma_{2}$, by assuming a decomposition $-P=s M+W$ and computing $\sigma_{2}(P)$. Multiplying $P$ on the left and right by $S O(3)$ matrices does not change the singular values, but does allow us to set $M=I$ and put $W$ into the form

$$
W=\left(\begin{array}{lll}
a & b & 0 \\
0 & 0 & 0 \\
0 & 0 & 0
\end{array}\right)
$$

Then

$$
P^{T} P=\left(\begin{array}{ccc}
(s+a)^{2} & (s+a) b & 0 \\
(s+a) b & s^{2}+b^{2} & 0 \\
0 & 0 & s^{2}
\end{array}\right)
$$

One of the eigenvalues of $P^{T} P$ is obviously $s^{2}$, with eigenvector $(0,0,1)$. Restricting to the upper left 2 by 2 block, we subtract $s^{2} I$ and get a matrix whose determinant, $-s^{2} b^{2}$, is nonpositive. Thus at most one eigenvalue of $P^{T} P$ is greater than $s^{2}$ and at most one eigenvalue is less than $s^{2}$. Since $s^{2}$ is the middle eigenvalue of $P^{T} P$, $\sigma_{2}(P)=s$.

Next we show that $P+s M$ can have rank 1 , with $s=\sigma_{2}(P)$, in two ways. By multiplying on the left and right by $S O(3)$ matrices, we can take $P$ diagonal, with entries $P_{11} \geq P_{22} \geq\left|P_{33}\right|$. Next we look for orthogonal matrices of the form

$$
M_{\theta}=\left(\begin{array}{ccc}
-\cos (\theta) & 0 & \sin (\theta) \\
0 & -1 & 0 \\
\sin (\theta) & 0 & \cos (\theta)
\end{array}\right)
$$

We then have

$$
P+s M_{\theta}=P+P_{22} M_{\theta}=\left(\begin{array}{ccc}
P_{11}-P_{22} \cos (\theta) & 0 & P_{22} \sin (\theta) \\
0 & 0 & 0 \\
P_{22} \sin (\theta) & 0 & P_{33}+P_{22} \cos (\theta)
\end{array}\right) .
$$

This matrix has an obvious null vector $(0,1,0) . P+s M_{\theta}$ has rank one (or zero) if, and only if, there is a second null vector. To see if there is a second null vector, we restrict $P+s M_{\theta}$ to the 1-3 plane and take its determinant, which equals $-P_{22}^{2}+$ $P_{11} P_{33}+\left(P_{11}-P_{33}\right) P_{22} \cos (\theta)$. This is a periodic function of $\theta$ with a single maximum of $\left(P_{11}-P_{22}\right)\left(P_{22}+P_{33}\right)$ at $\theta=0$ and a single minimum of $-\left(P_{11}+P_{22}\right)\left(P_{22}-P_{33}\right)$ at $\theta=\pi$. If $P_{11}>P_{22}>\left|P_{33}\right|$, the maximum and minimum values have opposite 
signs, so the function must cross zero exactly twice, at the points $\theta= \pm \cos ^{-1}\left(\left[P_{22}^{2}-\right.\right.$ $\left.\left.P_{11} P_{33}\right] /\left(P_{11}-P_{33}\right) P_{22}\right)$. If $P_{11}=P_{22}$ or $P_{33}=-P_{22}$, then the maximum value becomes zero, while if $P_{22}=P_{33}$ then the minimum becomes zero. In these cases we have a double root at $\theta=0$ or $\pi$. Finally, if $P_{11}=P_{22}=P_{33}$, then the function is identically zero and we have an infinite number of roots. This corresponds to the original $P$ being a positive multiple of an $S O(3)$ matrix.

Finally, we show that these are the only possible decompositions with $s=P_{22}$. Suppose that $M$ is an $S O(3)$ matrix with $P+s M$ having rank 1 . Then every 2 by 2 block of $P+s M$ has determinant 0 , and in particular the upper left 2 by 2 block has a null vector $v$. However, $P_{11}$ and $P_{22}$ are both at least $s$, so $|P v| \geq s$. The upper left corner of $s M$ has operator norm at most $s$, so $|s M v| \leq s$. Thus we must have $|P v|=s|M v|=s=P_{22}$. If $P_{11}>P_{22}$ this means $v=(0,1,0)$, so $M v=(0,-1,0)$, so $M$ must take the form (2.5). The case $P_{11}=P_{22}$ must be checked separately, but leads only to the solution $M=\operatorname{diag}(-1,-1,1)$.

The form of the explicit solutions found above also demonstrates the continuous dependence of $M$ on $P$. Expressed invariantly, $M$ is a rotation by $\pi$ about an axis. This axis is orthogonal to the second principal axis of $P^{T} P$, and makes an angle $\theta / 2=( \pm 1 / 2) \cos ^{-1}\left(\left[\sigma_{2}^{2} \pm \sigma_{1} \sigma_{3}\right] /\left[\left(\sigma_{1} \pm \sigma_{3}\right) \sigma_{2}\right]\right)$ with the third principal axis of $P^{T} P$, where the \pm is determined by the sign of the determinant of $P$. A small change in $P$ can only change $\theta$ by an amount of order $|\delta P| / \min \left(\sigma_{1}-\sigma_{2}, \sigma_{2}-\sigma_{3}\right)$, and, by first order perturbation theory (integrated to get rigorous bounds), can only change the principal axes of $P^{T} P$ by a similar amount. Thus if $\delta P$ is a small perturbation of $P$, the norm of the corresponding $\delta M$ is bounded by a constant times $|\delta P| / \min \left(\sigma_{1}-\sigma_{2}, \sigma_{2}-\sigma_{3}\right)$.

Not surprisingly, this stability breaks down when we approach the double root. If two of the singular values are equal, then a small perturbation may change $M$ by as much as $O(\sqrt{|\delta P|})$.

Proof of Theorem 2.1: Let $s_{p}$ be the second singular value of $F_{0}(p)$, and let $M_{p} \in S O(3)$ be a matrix such that $F_{0}(p)+s_{p} M_{p}$ is reducible (with similar definitions for $s_{q}$ and $M_{q}$ ). Let $s_{0}$ be the second singular value of $F_{0}(0)$. Note that $s_{0}>0$, since the three singular values of $F_{0}(0)$ were assumed distinct. Since $s_{p}$ and $s_{q}$ are within $O(L)$ of $s_{0}$, we can bound $s_{p}$ and $s_{q}$ away from zero.

We shall count the ways to simultaneously make $F_{\text {std }}(p)=s_{p} M_{p}$ and $F_{\text {std }}(q)=$ $s_{q} M_{q}$. The condition for the standard curvature $F_{\text {std }}$ to have magnitude $s_{p}$ at $p$ is

$$
\frac{\lambda^{2}}{\left(|y-p|^{2}+\lambda^{2}\right)^{2}}=s_{p},
$$

or equivalently

$$
\lambda^{2}+|y-p|^{2}=\lambda / \sqrt{s_{p}}
$$


As long as $|y-p|<1 / 2 \sqrt{s_{p}}$ there are two solutions to (2.8), while for $|y-p|>1 / 2 \sqrt{s_{p}}$ there are none. When $|y-p|<1 / 2 \sqrt{s_{p}}$, one solution has $\lambda>1 / 2 \sqrt{s_{p}}$, which is greater than $K L^{\alpha}$ for $L$ small. The other solution qualifies as small if $|y-p|$ is small enough, and, for $|y-p| \ll 1 / \sqrt{s_{p}}$, is approximately $\lambda=|y-p|^{2} \sqrt{s_{p}}$. As a set in $\mathbf{R}^{5}=(N, \lambda)$ space, the solutions to $(2.8)$ are a 4 -sphere. Projected onto $N$, they form (2 copies of) a 4-disk. In either case, only a small subset of solutions qualifies as "small".

The interesting question, of course, is how many times we can solve the equations for $p$ and $q$ simultaneously. We begin with equation (2.8) and the corresponding equation for $q$. The intersection of two 4 -spheres in $\mathbf{R}^{5}$ is a 3 -sphere. Projected onto $N$ we get a 3-dimensional ellipsoid, possible degenerating to two disks. As before, only a small patch of the ellipsoid (or alternatively part of one of the two disks) gives a small enough value of $\lambda$. It is this region that we consider.

Recall that $p$ and $q$ are at $\pm L$, where we are using quaternionic coordinates. For $L$ small, $s_{q}=s_{p}+O(L)$. Let $s_{m}$ be such that $2 / \sqrt{s_{m}}=1 / \sqrt{s_{p}}+1 / \sqrt{s_{q}}$. Let $\Delta=\left(1 / \sqrt{s_{p}}-1 / \sqrt{s_{q}}\right) / L$. As $L \rightarrow 0, s_{m}=s_{0}+O\left(L^{2}\right)$, while $\Delta$ approaches $-\left.\left(d s_{p} / d L\right)\right|_{L=0} / s_{0}^{3 / 2}$. Let $y_{0}$ and $y_{I}$ be the real and imaginary parts of $y$. Adding and subtracting (2.8) to the corresponding equation for $q$ we obtain

$$
-4 y_{0}=\lambda \Delta ; \quad \lambda^{2}+L^{2}+y_{0}^{2}+\left|y_{I}\right|^{2}=\lambda / \sqrt{s_{m}} .
$$

Plugging the first equation into the second we get

$$
\lambda^{2}\left(1+\frac{\Delta^{2}}{16}\right)-\frac{\lambda}{\sqrt{s_{m}}}+L^{2}+\left|y_{I}\right|^{2}=0
$$

This equation shows that $\lambda$, and thus $y_{0}$, may be viewed as functions of $y_{I}$. As long as $L^{2}+\left|y_{I}\right|^{2} \ll 1 / \sqrt{s_{m}}$ there are two solutions to $(2.10)$, one of which has $\lambda \approx$ $\left(L^{2}+\left|y_{I}\right|^{2}\right) \sqrt{s_{m}}$, the other of which has $\lambda \approx\left(\left(1+\Delta^{2} / 16\right) \sqrt{s_{m}}\right)^{-1}$. The first solution has $\lambda<K L^{\alpha}$ if and only if $\left|y_{I}\right|$ is small enough, while the second solution always has $\lambda>K L^{\alpha}$. Let $R_{K, \alpha}$ be the largest number such that $\left|y_{I}\right|<R_{K, \alpha}$ implies $\lambda \leq K L^{\alpha}$. Henceforth we consider only "admissible" $y$, i.e. those with $\left|y_{I}\right|<R_{K, \alpha}$. For $L$ chosen small enough, as we assume henceforth it is, $R_{K, \alpha}^{2} \sim K L^{\alpha} / \sqrt{s_{m}}-L^{2} \sim K L^{\alpha} / \sqrt{s_{m}}$, since $\alpha<2$. Note that

$$
y_{0}=-\lambda \Delta / 4 \approx-\left(L^{2}+\left|y_{I}\right|^{2}\right) \sqrt{s_{m}} \Delta / 4
$$

Hence for admissible $y$, we have $\left|y_{I}\right|<\operatorname{const} L^{\alpha / 2}$ and $\left|y_{0}\right|<\operatorname{const} L^{\alpha}$. Let $r=$ $\left(y_{0}(0), 0\right)$ be the unique admissible point where the ellipsoid of solutions $\left(y_{0}, y_{I}\right)$ to (2.9) hits the real axis. Since $|r|=O\left(L^{2}\right), r$ lies on the line segment $\overline{p q}$, and the ellipsoid has curvature $O(1)$ at $r$. See Figure 1. 


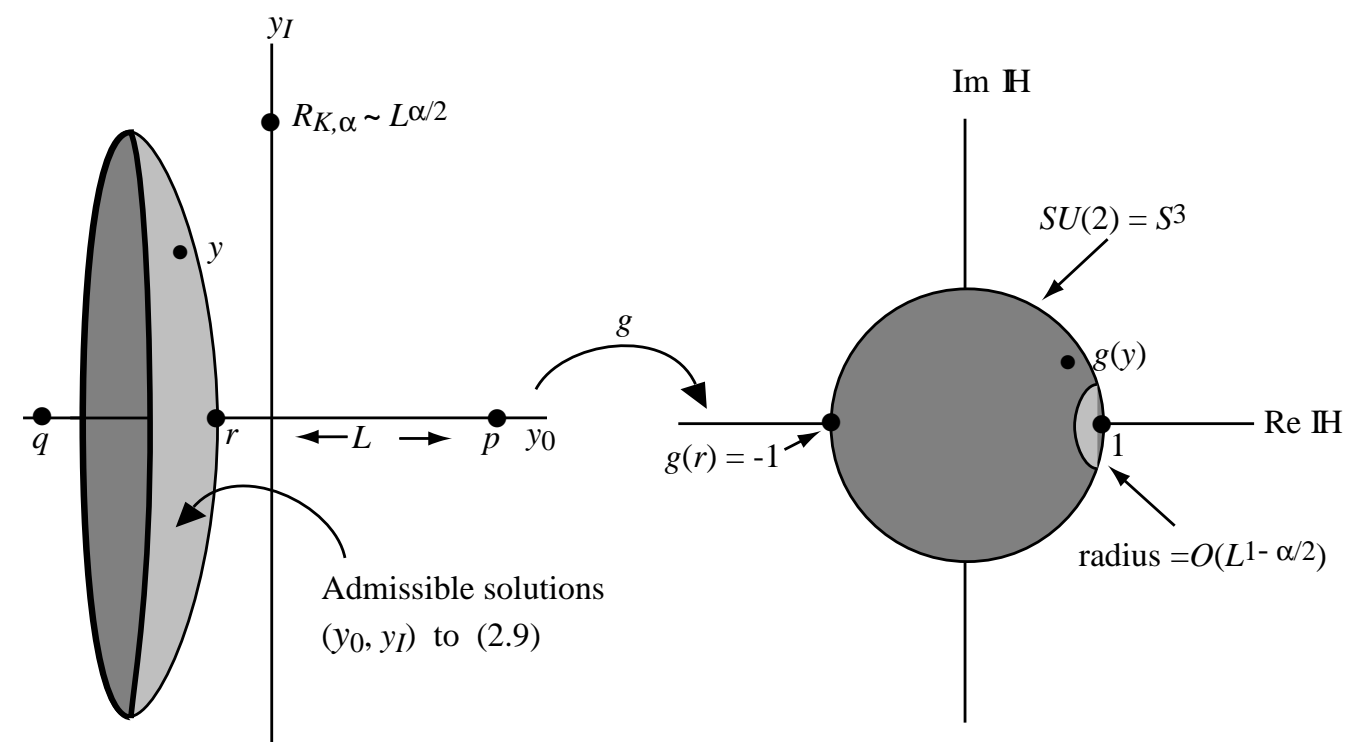

Figure 1

We still have to get the $S O(3)$ matrices right. This means simultaneously solving the equations $m^{-1} \rho((y-p) /|y-p|)=M_{p}$ and $m^{-1} \rho((y-q) /|y-q|)=M_{q}$ for $m$. If a solution exists it is obviously unique. A solution exists if and only if $\rho((y-p) / \mid y-$ $p \mid)^{-1} \rho((y-q) /|y-q|)=M_{p}^{-1} M_{q}$. Let $g(y)=(\bar{y}-\bar{p})(y-q) /|(y-p)(y-q)|$. We must count the points on our 3-disk (of small solutions to (2.9) and (2.10)) for which the $S O(3)$-valued function $\rho(g(y))$ equals $M_{p}^{-1} M_{q}$. Note that

$$
g(y)=-I+2 \frac{y_{I}}{L}\left(1+O\left(\left(\left|y_{0}\right| / L\right)^{2}\right)\right)+O\left(\left(\left|y_{I}\right| / L\right)^{2}\right) \quad \text { for }\left|y_{I}\right| \ll L,
$$

while

$$
g(y)=I+2 \frac{L y_{I}}{\left|y_{I}\right|^{2}}\left(1+O\left(\left(\left|y_{0}\right| /\left|y_{I}\right|\right)^{2}\right)\right)+O\left(\left(L /\left|y_{I}\right|\right)^{2}\right) \quad \text { for }\left|y_{I}\right| \gg L .
$$

In view of (2.11), we can replace $O\left(\left(\left|y_{0}\right| / L\right)^{2}\right)$ in $(2.12)$ and $O\left(\left(\left|y_{0}\right| /\left|y_{I}\right|\right)^{2}\right)$ in $(2.13)$ by $O\left(L^{2}\right)$ and $O\left(L^{2 \alpha}\right)$, respectively.

Observe that $L / R_{K, \alpha}$ is $O\left(L^{1-\alpha / 2}\right)$ and hence goes to zero as $L \rightarrow 0$. On the disk of admissible $y_{I}$, the map $g$ covers all of $S U(2)$ except for a ball of radius $c L^{1-\alpha / 2}$ around the identity, for some constant $c$. Since $\rho$ is a 2-1 map, $\rho(g(y))$ hits all of $S O(3)$ twice, except for a ball of radius $2 c L^{1-\alpha / 2}+O\left(L^{2-\alpha}\right)$ around the identity, which is only hit once. The number of solutions to our problem depends on whether, for small $L, M_{p}^{-1} M_{q}$ is in this ball or not.

If the singular values of $F_{0}(0)$ are distinct, then, by Lemma 2.2, there are two distinct matrices $M_{1,2}(0)$ for which $F_{0}(0)+\sigma_{2}(0) M$ has rank 1. By the comment 
after the proof of Lemma 2.2, the two matrices for $p$ and $q$ satisfy $M_{1,2}(p, q)=$ $M_{1,2}(0)+O(L)$. As $L \rightarrow 0, M_{1}(p)^{-1} M_{2}(q)$ and $M_{2}(p)^{-1} M_{1}(q)$ are bounded away from the identity, but $M_{1}(p)^{-1} M_{1}(q)$ and $M_{2}(p)^{-1} M_{2}(q)$ are within $O(L)$ (and hence within $\left.2 c L^{1-\alpha / 2}+O\left(L^{2-\alpha}\right)\right)$ of the identity. Thus we have two configurations in $(y, \lambda, m)$ space that give $s_{p} M_{1}(p)$ at $p$ and $s_{q} M_{2}(q)$ at $q$, two that give $s_{p} M_{2}(p)$ at $p$ and $s_{q} M_{1}(q)$ at $q$, one that gives $s_{p} M_{1}(p)$ at $p$ and $s_{q} M_{1}(q)$ at $q$ and one that gives $s_{p} M_{2}(p)$ at $p$ and $s_{q} M_{2}(q)$ at $q$. A total of six solutions in all.

On a codimension- 2 set of background data, the background curvature at the origin has two equal singular values, so $M_{1}(0)=M_{2}(0)$ and $M_{1,2}(p, q)=M_{1}(0)+$ $O\left(L^{1 / 2}\right)$. In that case all 4 possibilities have $M_{p}^{-1} M_{q}=1+O\left(L^{1 / 2}\right)$. If $\alpha>1$, this is within $2 c L^{1-\alpha / 2}$ of the identity for small enough $L$, and so each possibility yields one solution. If $\alpha<1$, and the $O\left(L^{1 / 2}\right)$ term in the expansion of $M_{1,2}(p, q)$ in powers of $L$ is nonzero, then each possibility yields two solutions.

Finally we consider the orientations of our solutions. It is not immediately clear that all solutions have the same orientation, but in fact they do. The problem of matching amplitudes is the same in all cases. The problem of matching gluing angles reduces to the intersection of two 3-cycles in a 3-disk $\times S O(3)$ (i.e., all possible pairs $\left.\left(y_{I}, m\right)\right)$, and is easily seen to be transverse. The intersection numbers are continuous functions of $M_{p}$ and $M_{q}$, as long as a solution continues to exist. Sending $M_{p}$ around a noncontractible loop in $S O(3)$ interchanges the two solutions associated to a given pair $\left(M_{p}, M_{q}\right)$, which shows that the two solutions for any given $\left(M_{p}, M_{q}\right)$ have the same orientation. Also by continuity, this orientation is the same for all pairs $\left(M_{p}, M_{q}\right)$.

All that remains is to compute this orientation in one case. Let $s_{p}=s_{q}=1$, $M_{p}=M_{q}=I$, and look near the solution with $y=0$ and $m=I$. The varieties $\tilde{\nu}_{p}$ and $\tilde{\nu}_{q}$ are just the zero sets of $F_{\text {std }}(p)-I$ and $\tilde{F}_{\text {std }}(q)-I$, which we view as functions of $(y, \lambda, m)$. Taking derivatives we find that changes in $(y, \lambda, m)$ give the following first order changes in $F_{\text {std }}(p)$ and $F_{\text {std }}(q)$ :

1. Increasing $\lambda$ increases the magnitude of both $\tilde{F}_{\text {std }}(p)$ and $F_{\text {std }}(q)$ without changing either direction.

2. Increasing $y_{0}$ increases the magnitude of $F_{\text {std }}(p)$ and decreases that of $F_{\text {std }}(q)$, while keeping the directions fixed.

3. Increasing $y_{1}$ (resp. $\left.y_{2}, y_{3}\right)$ rotates $\tilde{F}_{\text {std }}(p)$ in the direction defined by the Lie algebra element $-i$ (resp. $-j,-k)$, and rotates $F_{\text {std }}(p)$ an equal amount in the direction $+i$ (resp. $+j,+k)$.

4. Rotating $m$ in any direction rotates both $F_{\text {std }}(p)$ and $F_{\text {std }}(q)$ in the opposite direction.

From this we deduce that the Jacobian $\left|d\left(F_{\text {std }}(p), F_{\text {std }}(q)\right) / d(y, \lambda, m)\right|$ is positive, and so that the local intersection number of $\tilde{\nu}_{p}$ and $\tilde{\nu}_{q}$ is +1 in this case. Thus the 
local intersection number of $\tilde{\nu}_{p}$ and $\tilde{\nu}_{q}$ is +1 in all cases.

Having proven Theorem 2.1, we consider the question of stability. How much do our intersection points move around if we change $M_{p}$ or $M_{q}$ or $s_{p}$ or $s_{q}$ slightly? Since $F_{0}+F_{\text {std }}$ is only an approximation to the true curvature of a connection in $\mathcal{M}_{k+1}$, our results must be stable in order to be meaningful.

Let $\chi$ be the map that takes $(y, \lambda, m)$ to $\left(F_{\text {std }}(p), F_{\text {std }}(q)\right)$. Near our solutions, $d \chi$ is never close to singular. By changing $\lambda$ and one component of $y$ we can adjust $\left|F_{\text {std }}(p)\right|$ and $\left|F_{\text {std }}(q)\right|$ independently, while by adjusting $m$ and the remaining three components of $y$ we can adjust the directions of $F_{\text {std }}(p)$ and $F_{\text {std }}(q)$ independently. It is not difficult to estimate the matrix elements of $(d \chi)^{-1}$. Some are $O(1)$, some are $O(L)$, and some are $O\left(L^{2}\right)$. If we know the required $F_{\text {std }}(p$ or $q)$ to within $\epsilon$, we know $m$ to within $O(\epsilon), y$ to within $O(\epsilon L)$, and $\lambda$ to within $O\left(\epsilon L^{2}\right)$. In short, small errors in the input data result in only small changes of the locations of our intersection points in $(y, \lambda, m)$ space.

Finally, we consider a perturbation of our model problem that is more directly applicable in the sequel. Let $\tilde{F}_{0}(x)$ be the curvature of the background connection in the standard radial gauge about the gluing point $y$. (That is, use the original connection $A_{0}$ to trivialize the fiber over $y$, and then use parallel transport radially outwards from $y$ to trivialize the bundle over $U$.) We wish to count the number of ways to make $\tilde{F}_{0}+F_{\text {std }}$ reducible at both $p$ and $q$.

Theorem 2.3 Under the assumptions of Theorem 2.1, the number of ways to make $\tilde{F}_{0}+F_{\text {std }}$ reducible at $p$ and $q$ (counted with sign) is the same as the number of ways to make $F_{0}+F_{\text {std }}$ reducible at $p$ and $q$ (counted with sign), namely +6 .

Proof: We first put our background connection into a radial gauge with respect to the origin. This is a fixed gauge, and Theorem 2.1 applies. Since $F_{0}$ and $\tilde{F}_{0}$ are related by a gauge transformation, the singular values of $F_{0}$ and $\tilde{F}_{0}$ are the same. Thus we must solve (2.8), and the corresponding equation for $q$, exactly as in Theorem 2.1, with the same values of $s_{p}$ and $s_{q}$. We then solve $m^{-1} \rho((y-p) /|y-p|)=M_{p}$ and $m^{-1} \rho((y-q) /|y-q|)=M_{q}$ for $m$, as before. The only difference in our analysis is that $M_{p}$ and $M_{q}$ are now functions of $y$. We compute the extent to which they depend on $y$.

Let $A$ be a connection in radial gauge with respect to $y$, and let $A^{\prime}$ be the same connection in radial gauge with respect to $y^{\prime}$. The gauge transformation that relates these, evaluated at the point $p$, is the holonomy around a triangle from $p$ to $y$ to $y^{\prime}$ to $p$, and so its difference from the identity is bounded by the sup norm of $\left|F_{A}\right|$ times the area of the triangle. See Figure 2. 


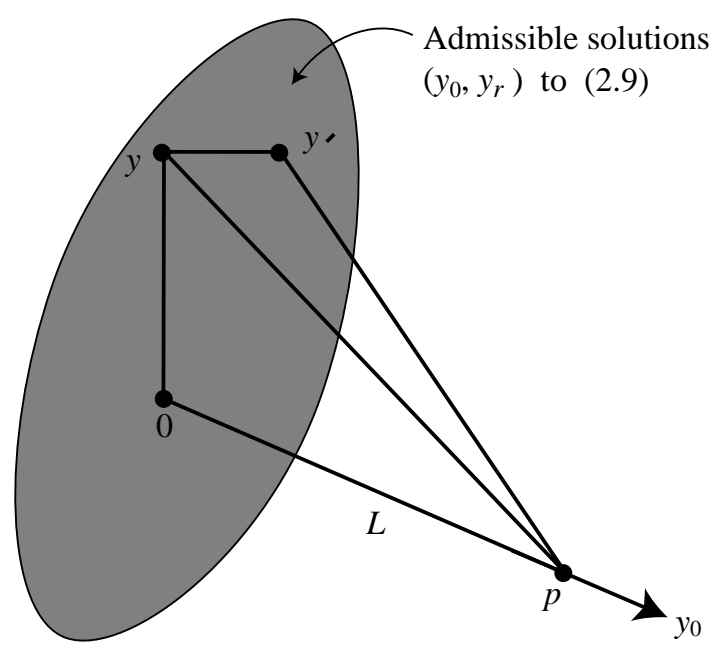

Figure 2

In our case, $A$ is the background connection, so $\left|F_{A}\right|$ is fixed and bounded, and $y$ and $y^{\prime}$ are restricted to lie on the ellipsoid of solutions to (2.9) and (2.10), with $\left|y_{I}\right|$ and $\left|y_{I}^{\prime}\right|$ both less than $R_{K, \alpha}$. Note that the area of a triangle is bounded by half the product of the length of any two of its legs. Because the curvature of the ellipsoid of solutions $y=\left(y_{0}, y_{I}\right)$ is $O(1)$ at admissible points, $\left|y_{0}-y_{0}^{\prime}\right|$ is bounded by a constant times $\left|y_{I}-y_{I}^{\prime}\right|$. As a result,

$$
\left|M_{p}(y)-M_{p}\left(y^{\prime}\right)\right| \leq \text { const } \times \sqrt{L^{2}+|y|^{2}}\left|y_{I}-y_{I}^{\prime}\right|,
$$

while

$$
\left|M_{p}(y)-M_{p}(0)\right| \leq \text { const } \times L\left|y_{I}\right|,
$$

with similar estimates for $M_{q}$. The second result is an estimate on $M_{p}$ itself, while the first leads to a bound on the derivative of $M$ with respect to $y_{I}$. By (2.11), $L^{2}+|y|^{2} \leq$ const $\left(L^{2}+\left|y_{I}\right|^{2}\right)$, so we obtain

$$
\left|\partial M_{p} / \partial y_{I}\right| \leq \text { const } \times \sqrt{L^{2}+\left|y_{I}\right|^{2}} .
$$

As before, we look for solutions to $\rho(g(y))=M_{p}^{-1} M_{q}$, where now the right hand side depends on $y$. We break the disk of radius $R_{K, \alpha}=O\left(L^{\alpha / 2}\right)$ into two pieces, a inner disk $D_{1}$ and an annulus $D_{2}$. The radii will be chosen such that on $D_{1}$ the estimate (2.14) is strong enough to allow implicit function theorem arguments to apply. Here the solutions to $\rho(g(y))=M_{p}^{-1}(y) M_{q}(y)$ are but small perturbations of the solutions to $\rho(g(y))=M_{p}^{-1}(0) M_{q}(0)$. On $D_{2}$ the estimate (2.15) will be strong enough to show that there are no solutions to $\rho(g(y))=M_{p}^{-1}(y) M_{q}(y)$. Taken together, this will prove the theorem.

On the disk $D_{1}$, the implicit function theorem will apply as long as the smallest singular value of $\partial(\rho \circ g) / \partial y_{I}$ is at least twice the largest singular value of 
$\left.\partial\left(M_{p}^{-1} M_{q}\right)\right) / \partial y_{I}$, which by (2.16) is bounded above by a multiple of $\left(L^{2}+\left|y_{I}\right|^{2}\right)^{1 / 2}$. Computing the derivative of $\rho \circ g$ is an easy geometrical calculation, and one finds that all singular values are bounded below by a constant times $L /\left(L^{2}+\left|y_{I}\right|^{2}\right)$. Comparing $L /\left(L^{2}+\left|y_{I}\right|^{2}\right)$ to $\left(L^{2}+\left|y_{I}\right|^{2}\right)^{1 / 2}$ we see that the implicit function theorem applies whenever $\left|y_{I}\right|$ is smaller than a constant times $L^{1 / 3}$, and in particular whenever $\left|y_{I}\right|<L^{1 / 2}$ (and $L$ is sufficiently small). We take the radius of $D_{1}$ (and the inner radius of $\left.D_{2}\right)$ to be $L^{1 / 2}$.

Now consider $y_{I} \in D_{2}$. If $\alpha>1$, then $D_{2}$ is empty, so we assume $\alpha \leq 1$. By (2.13), $|I-\rho(g(y))|=2 L /\left|y_{I}\right|\left(1+O\left(L^{\alpha}\right)\right)+O\left(L^{2} /\left|y_{I}\right|^{2}\right)$. Since $c_{1} L^{1 / 2}<\left|y_{I}\right|<c_{2} L^{\alpha / 2}$, $c_{3} L^{1-\alpha / 2}<|I-\rho(g(y))|<c_{4} L^{1 / 2}$. Now recall that $M_{p}^{-1}(0) M_{q}(0)$ is either bounded away from the identity or is within $O(L)$ of the identity (e.g. $M_{1}^{-1}(p) M_{2}(q)$ is bounded away from the identity while $M_{1}^{-1}(p) M_{1}(q)$ is within $O(L)$ of the identity). By (2.15), $M_{p}^{-1}(y) M_{q}(y)$ is also either bounded away from the identity or within $O(L)$ of the identity on $D_{2}$. Thus $\left|I-M_{p}^{-1}(y) M_{q}(y)\right|$ can never be between $c_{3} L^{1-\alpha / 2}$ and $c_{4} L^{1 / 2}$, so there are no solutions to $\rho(g(y))=M_{p}^{-1}(y) M_{q}(y)$ on $D_{2}$.

\section{The Perturbed Moduli Space}

In this section we show that the model problem of $\S 2$ correctly describes the intersection of $\nu_{p}, \nu_{q}$, and a perturbation (denoted $\tilde{\mathcal{M}}_{k+1}$ ) of the boundary region of $\mathcal{M}_{k+1}$. $\tilde{\mathcal{M}}_{k+1}$ is parametrized by quadruples $\left(A_{0}, y, \lambda, m\right)$, where $A_{0} \in \mathcal{M}_{k}$ is a background connection, and the glued-in bubble has size $\lambda$, center $y$ and gluing angle $m$. We construct $\tilde{\mathcal{M}}_{k+1}$ by an explicit gluing formula and show that, in the relevant region, the curvature of a connection in $\tilde{\mathcal{M}}_{k+1}$ is well approximated by the sum of the background curvature $F_{0}$ and the curvature $F_{\text {std }}$ of a standard instanton of size $\lambda$, center $y$ and gluing angle $m$. Our model problem was essentially to make this sum reducible at $p$ and $q$. By interpolating between this sum and the actual curvature of a connection in $\tilde{\mathcal{M}}_{k+1}$, we show that the results of $\S 2$ carry over almost word for word.

As before, we pick a background connection $A_{0} \in \mathcal{M}_{k}$ and constants $K>0$ and $\alpha \in(0,2)$. Let the neighborhood $\tilde{U}$ in $N$, and the corresponding neighborhood $U$ of the origin in $\mathbf{R}^{4}$, be as in $\S 2$. We now allow bubbles to be glued in anywhere (not just in $\tilde{U})$, so the set $B$ of gluing data is a $\left(0, K L^{\alpha}\right) \times S O(3)$ bundle over $N$, with local coordinates $(y, \lambda, m) \in N \times\left(0, K L^{\alpha}\right) \times S O(3)$. When the center of the bubble is in $\tilde{U}$, we identify the center point in $N$ with the corresponding coordinate in $U$, and call both points $y$. For each $(y, \lambda, m) \in B$, let $F$ be the curvature of the connection $\left(A_{0}, y, \lambda, m\right) \in \tilde{\mathcal{M}}_{k+1}$. The variety $\nu_{p}$ (resp. $\left.\nu_{q}\right)$, restricted to the fiber of $\tilde{\mathcal{M}}_{k+1}$ over $A_{0}$, is the set of points $(\lambda, y, m) \in B$ such that $F^{-}(p)$ (resp. $\left.F^{-}(q)\right)$ is reducible. We must count the intersection points of $\nu_{p}$ and $\nu_{q}$. In this section we prove 
Theorem 3.1 Fix $K>0, \alpha \in(0,2)$, and $A_{0} \in \mathcal{M}_{k}$. If the singular values of $F_{A_{0}}(0)$ are all distinct, then, for all sufficiently small $L$, the intersection number of $\nu_{p}, \nu_{q}$, and the fiber of $\tilde{\mathcal{M}}_{k+1}$ over $A_{0}$ is +6 .

We begin by constructing the space $\tilde{\mathcal{M}}_{k+1}$. For now, assume we are gluing a bubble of size $\lambda$ in $\tilde{U}$, with the center point at the origin. There are three natural length scales determined by the background connection $A_{0}$. The first is the length scale $\left|F_{A_{0}}(0)\right|^{-1 / 2}$ of the background curvature at the origin. The second is the length scale $\left|F_{A_{0}}(0)\right| /\left|\nabla^{A} F_{A_{0}}(0)\right|$ at which this curvature varies. Let $R_{3}$ be the smaller of these two length scales. Finally, let $s_{0}$ be the second singular value of $F_{A_{0}}(0)$. It is easy to see that $s_{0}<1 / R_{3}^{2}$, but there is no simple lower bound for $s_{0}$ (although, by assumption, $s_{0}$ is always positive). As we have seen, $s_{0}$ measures how far $F_{A_{0}}(0)$ is from being reducible.

Now pick additional length scales $R_{1}$ and $R_{2}$, which can depend on $\lambda, R_{3}$ and $s_{0}$, such that $R_{1}^{2}<10^{-6} \lambda R_{3}$ and $R_{2}^{2}>10^{6} \lambda / \sqrt{s_{0}}$. When $\lambda \ll R_{3}$, which is the only case we will consider, we want $\lambda \ll R_{1} \ll R_{2} \ll R_{3}$. The points of interest $x$ will all have $R_{1}<|x|<R_{2}$. The number $10^{6}$ is of course arbitrary. It is just chosen large enough that we can safely ignore small numerical factors.

Let $\beta(r)$ be a smooth monotonic function that equals zero for $r<1 / 2$ and equals 1 for $r>2$, and such that $\beta^{\prime} \leq 1$. We define cutoff functions $\beta_{1}(x)=\beta\left(|x| / R_{1}\right)$ and $\beta_{2}(x)=1-\beta\left(|x| / R_{2}\right)$.

Let $A_{0}$ be the background connection expressed in a smooth fixed radial gauge with respect to the origin. Let $A_{\text {std }}$ be the connection of a standard instanton of size $\lambda$ expressed in a radial gauge that is singular at the origin and regular at $\infty$. (This gauge is not unique; it depends on a gluing angle $m$. See the discussion before expression (2.2)). Note that $\left|A_{\text {std }}\right| \sim \lambda^{2} / r^{3}$ for $r \gg \lambda$ while $\left|A_{0}\right| \sim r\left|F_{A_{0}}\right|=r / R_{3}^{2}$ for $r \ll R_{3}$.

Our point $\left(A_{0}, 0, \lambda, m\right) \in \tilde{\mathcal{M}}_{k+1}$ is defined by the connection form

$$
A^{\prime}=\beta_{1} A_{0}+\beta_{2} A_{\text {std }} .
$$

We compute

$$
\begin{aligned}
F=F_{A^{\prime}}=d A^{\prime}+A^{\prime} \wedge A^{\prime} & =\beta_{1} F_{A_{0}}+\beta_{2} F_{A_{\text {std }}} \\
& +\left(\beta_{1}^{2}-\beta_{1}\right) A_{0} \wedge A_{0}+\left(\beta_{2}^{2}-\beta_{2}\right) A_{\text {std }} \wedge A_{\text {std }} \\
& +d \beta_{1} \wedge A_{0}+d \beta_{2} \wedge A_{\text {std }} \\
& +\beta_{1} \beta_{2}\left(A_{\text {std }} \wedge A_{0}+A_{0} \wedge A_{\text {std }}\right),
\end{aligned}
$$

and the interpolating 2 -form

$$
F_{t}=t\left(F_{A_{0}}+F_{\text {std }}\right)+(1-t) F,
$$


where $0 \leq t \leq 1$

If the bubble is to be glued in at a point $y$, rather than at the origin, we must adjust the formulas as follows. First suppose $y \in \tilde{U}$. Take $A_{0}$ as the connection of the background in radial gauge with respect to $y$ (not with respect to 0 ). The quantities $s_{0}, R_{1}, R_{2}$, and $R_{3}$ are computed from the curvature $F_{A_{0}}(y)$, not $F_{A_{0}}(0)$. The connection $A_{\text {std }}$ is in a singular radial gauge with respect to $y$ (not to 0 ). The cutoff functions are $\beta_{1}(x)=\beta\left(|x-y| / R_{1}\right)$ and $\beta_{2}(x)=1-\beta\left(|x-y| / R_{2}\right)$. With these modifications, we still have $A^{\prime}=\beta_{1} A_{0}+\beta_{2} A_{\text {std }}$, and formulas (3.2) and (3.3) still apply. For $y \notin U$, just apply the same formulas, using geodesic normal coordinates around $y$. In this case the "standard instanton" $A_{\text {std }}$ is no longer exactly anti-selfdual, but becomes anti-self-dual in the $\lambda \rightarrow 0$ limit. The gluing angle $m$ depends on a local trivialization, but the set of gluing angles is invariant. This defines the space $\tilde{\mathcal{M}}_{k+1}$ for all $y$.

In $\S 2$ we distinguished notationally between radial gauge with respect to 0 and radial gauge with respect to $y$, calling the background curvature $F_{0}$ in the first case and $\tilde{F}_{0}$ in the second case. Theorem 2.1 discussed making $F_{0}+F_{\text {std }}$ reducible at $p$ and $q$, while Theorem 2.3 discussed making $\tilde{F}_{0}+F_{\text {std }}$ reducible at $p$ and $q$. In this section the background connection is always in radial gauge with respect to the gluing point $y$. With only one case to consider, we always write $F_{0}$, never $\tilde{F}_{0}$.

Note that we do not use the gluing formula found in standards works such as [DK]. Traditionally, one takes $A^{\prime \prime}=\left(1-\beta_{2}\right) A_{0}+\left(1-\beta_{1}\right) A_{\text {std }}$, so that the resulting connection is exactly flat in the annulus with radii $2 R_{1}$ and $R_{2} / 2$ around $y$. This makes identifying the bundles on which $A_{0}$ and $A_{\text {std }}$ live conceptually easier. However, such a procedure makes for a perturbed moduli space on which $\nu_{p}$ and $\nu_{q}$ intersect nontransverely, since $F_{A^{\prime \prime}}^{-}$is reducible, indeed zero, on the entire annulus $2 R_{1}<r<R_{2} / 2$. This makes the intersection number effectively impossible to compute.

Instead, we allow the supports of $\beta_{1} A_{0}$ and $\beta_{2} A_{\text {std }}$ to overlap, as in Taubes' work such as $[\mathrm{T}]$. This allows us to observe the interaction between the background connection and the glued-in instanton. In the $[\mathrm{DK}]$ method, the interaction only occurs when we go from our explicit approximate ASD connection to the true ASD connection (something we have relatively little analytic control over). In our method, the interaction is seen at the level of the approximate connection $A^{\prime}$, which we can calculate. Moreover, $F_{A^{\prime}}^{+}$is much smaller than $F_{A^{\prime \prime}}^{+}$(in the $L^{2}$ norm), so our method should give a closer approximation to the properties of the true moduli space.

Let $\nu_{t, p}\left(\operatorname{resp} . \nu_{t, q}\right)$ be the set of gluing data $(y, \lambda, m)$ with $\lambda<K L^{\alpha}$ for which $F_{t}^{-}(p)$ (resp. $\left.F_{t}^{-}(q)\right)$ is reducible. If $y$ is not in $\tilde{U}$, then, for small enough $\lambda$, the connection form near $p$ is exactly $A_{0}$. By assumption, $F_{0}$ is not reducible at the origin. For small enough $L$, therefore, $F_{0}$ is not reducible at $p$, and $F_{t}(p)=F_{0}(p)$ is not reducible. We may therefore assume, without loss of generality, that our gluing point $y$ is always in $\tilde{U}$. Indeed, by picking $L$ small enough we may assume that $y$ is in 
an arbitrarily small neighborhood of the origin, and therefore that $F_{0}(y)$ is arbitrarily close to $F_{0}(0)$. Thus we may take the length scales $R_{1}, R_{2}$, and $R_{3}$ to be independent of $y$ (although $R_{1}$ and $R_{2}$ may depend on $\lambda$ ).

We consider five possibilities:

1. I. $|p-y| \leq R_{1} / 2$, ( $p$ is in the "interior zone", where $\beta_{1}=0$ and $\left.\beta_{2}=1\right)$,

2. II. $R_{1} / 2<|p-y|<2 R_{1},(p$ is in the interior "shoulder"),

3. III. $2 R_{1} \leq|p-y| \leq R_{2} / 2$, ( $p$ is in the "plateau", where $\left.\beta_{1}=\beta_{2}=1\right)$,

4. IV. $R_{2} / 2<|p-y|<2 R_{2}$, ( $p$ is in the exterior "shoulder"), and

5. V. $|p-y| \geq 2 R_{2}$, ( $p$ is in the "exterior zone", where $\beta_{1}=1$ and $\left.\beta_{2}=0\right)$.

As in $\S 2$, we will be identifying curvatures with $3 \times 3$ real matrices. The phrase "the second singular value of $F$ ", for example, is shorthand for "the second singular value of $\operatorname{Mat}\left(F^{-}\right)$".

The problem of Theorem 2.3 was to find $\nu_{1, p}, \nu_{1, q}$ and count their intersection points. In that problem condition III always applied, with $|p-y|^{2} \approx \lambda / \sqrt{s_{p}}$. We will show that $F_{t}^{-}(p)$ being reducible with $\lambda<K L^{\alpha}$ also implies condition III, and that $\nu_{t, p}$ is a small perturbations of $\nu_{1, p}$. We establish condition III by showing that the other conditions lead to contradictions.

We begin by considering condition I. Where $|x-y| \leq R_{1} / 2, A^{\prime}=A_{\text {std }}$ has ASD curvature, so $F_{t}^{-}=F_{t}=t F_{A_{0}}+F_{\text {std }}$. For $F_{t}$ to be reducible at $p$ we need $\left|F_{\text {std }}(p)\right|=t s_{p}$. That is, $\lambda^{2}+|p-y|^{2}=\lambda / \sqrt{t s_{p}}$. This quadratic equation has two solutions, one with $\lambda \approx|p-y|^{2} \sqrt{t s_{p}}$, the other with $\lambda \approx 1 / \sqrt{t s_{p}}$, but neither is consistent with condition I. Since $|p-y|<R_{3}, s_{p}$ is close to $s_{0}$. Since $|p-y|^{2}<R_{1}^{2}<10^{-6} \lambda R_{3}$, while $\sqrt{t s_{0}} \leq 1 / R_{3}$, one cannot have $\lambda \approx|p-y|^{2} \sqrt{t s_{p}}$. The second solution has $\lambda \approx 1 / \sqrt{t s_{p}}>R_{3}$, which contradicts $\lambda \ll R_{3}$. Thus condition I is impossible.

If $p$ is in the interior shoulder we have additional terms to consider:

$F_{t}=F_{\text {std }}+\left(t+(1-t) \beta_{1}\right) F_{A_{0}}+(1-t)\left[\left(\beta_{1}^{2}-\beta_{1}\right) A_{0} \wedge A_{0}+d \beta_{1} \wedge A_{0}+\beta_{1}\left(A_{\text {std }} \wedge A_{0}+A_{0} \wedge A_{\text {std }}\right)\right]$.

The ASD part of the terms after $F_{\text {std }}$ can be bounded in norm by $1 / R_{3}^{2}+4 R_{1} / R_{3}^{4}+$ $4 / R_{3}^{2}+\lambda^{2} / R_{1}^{2} R_{3}^{2}<100 / R_{3}^{2}$, and so the second singular value of $F_{t}$ is within $100 / R_{3}^{2}$ of the second singular value of $F_{\text {std }}$. For $F_{t}^{-}$to be reducible, $\left|F_{\text {std }}\right|$ can be at most $100 / R_{3}^{2}$. Thus we need $\lambda /\left(\lambda^{2}+|p-y|^{2}\right)<10 / R_{3}$, which in turn means that either $\lambda>R_{3} / 100$ or $\lambda<100 R_{1}^{2} / R_{3}$. The first is not allowed, as $\lambda$ is assumed small. The second contradicts the definition of $R_{1}$. So condition II is also impossible.

If $p$ is in the exterior zone, we have $F_{t}=F_{A_{0}}+(1-t) F_{\text {std }}$, so we need $\lambda /\left(\lambda^{2}+\mid p-\right.$ $\left.\left.y\right|^{2}\right)=\sqrt{s_{p} /(1-t)}$, or equivalently $\lambda=\left(\lambda^{2}+|p-y|^{2}\right) \sqrt{s_{p} /(1-t)}$. But $|p-y|^{2}>$ 
$2 R_{2}^{2}>10^{6} \lambda / \sqrt{s_{0}}$, so $\sqrt{s_{p} /(1-t)}$ always exceeds $\lambda /\left(\lambda^{2}+|p-y|^{2}\right)$. So again we have a contradiction.

If $p$ is in the exterior shoulder, we have

$F_{t}=\left(t+(1-t) \beta_{2}\right) F_{\text {std }}+F_{A_{0}}+(1-t)\left[\left(\beta_{2}^{2}-\beta_{2}\right) A_{\text {std }} \wedge A_{\text {std }}+d \beta_{2} \wedge A_{\text {std }}+\beta_{2}\left(A_{\text {std }} \wedge A_{0}+A_{0} \wedge A_{\text {std }}\right)\right]$.

The ASD parts of the terms other than $F_{A_{0}}$ have total magnitude bounded by $\lambda^{2} / R_{2}^{4}+$ $\lambda^{4} / R_{2}^{6}+\lambda^{2} / R_{2}^{4}+\lambda^{2} / R_{2}^{2} R_{3}^{2}<10 \lambda^{2} / R_{2}^{4}<10^{-11} s_{0}<10^{-10} s_{p}$. But $F_{A_{0}}$ is a distance greater than $s_{p} / 2$ from the nearest reducible matrix, so $F_{t}^{-}(p)$ cannot be reducible.

Thus for all points in $\nu_{t, p}$ condition III applies, and here the analysis is relatively simple. The cutoff functions are both 1 , so $F(p)=F_{A_{0}}+F_{\text {std }}+(1-t)\left(A_{\text {std }} \wedge A_{0}+A_{0} \wedge\right.$ $\left.A_{\text {std }}\right)$. This last term has magnitude bounded by $\lambda^{2} / R_{1}^{2} R_{3}^{2}$, and changes only slightly as $(y, \lambda, m)$ are varied. It can thus be treated as a perturbation of $F_{A_{0}}$. We perturb $\nu_{1, p}$ to $\nu_{t, p}$ iteratively (as in the standard proof of the inverse function theorem): Given a point in $\nu_{1, p}$, compute $(1-t)\left(a \wedge A_{0}+A_{0} \wedge a\right)$, use that to adjust $(y, \lambda, m)$, compute the change in $(1-t)\left(A_{\text {std }} \wedge A_{0}+A_{0} \wedge a\right)$, adjust $(y, \lambda, m)$, and so on. The iteration converges geometrically. Similarly, a point in $\nu_{t, p}$ can be perturbed to a point in $\nu_{1, p}$.

Of course, the same analysis applies to $\nu_{t, q}$.

Now we consider the number of intersection points of $\nu_{t, p}$ and $\nu_{t, q}$, as a function of $t$. The only way the intersection number can change is if intersection points appear or disappeared at the ends of $\nu_{t, p}$ or $\nu_{t, q}$. However, we have shown that such intersection points can only occur when both $p$ and $q$ are in the plateau. In the proof of Theorem 2.3 we saw that, for $\lambda \gg L^{2}$ but $\lambda \ll 1$ (e.g., $\lambda \sim K L^{\alpha}$ ), the points of $\nu_{1, p}$ are bounded away from $\nu_{1, q}$. Since condition III applies, for $\lambda \sim K L^{\alpha}, \nu_{t, p}$ and $\nu_{t, q}$ are close to $\nu_{1, p}$ and $\nu_{1, q}$, respectively, and so are bounded away from each other. Thus intersection points between $\nu_{t, p}$ and $\nu_{t, q}$ may not appear from or disappear to the boundary. Thus $\#\left(\nu_{0, p} \cap \nu_{0, q}\right)=\#\left(\nu_{1, p} \cap \nu_{1, q}\right)$. By Theorem 2.3 , the latter number is +6 , regardless of $A_{0}$.

\section{Computing the Donaldson Invariants}

In sections 2 and 3 we saw that, for a fixed generic background connection, there are six ways to glue in a small bubble near $p$ and $q$ so as to make the curvature reducible at $p$ and $q$. In this section we demonstrate that this is sufficient information to compute the contribution of the boundary region of $\tilde{\mathcal{M}}_{k+1}$ to the simple type condition. For generic choices of representatives (of the classes other than $\mu(p)$ and $\mu(q)$ ), and for generic choice of the location of the origin of our coordinate system, the boundary region contributes $6 / 64$ of what is needed for simple type. 
We continue the notation of $\S \S 2-3 . \tilde{\mathcal{M}}_{k+1}$ is the perturbed moduli space and $\tilde{U}$ is a fixed ball in $N$ with a Euclidean metric, which we identify with a neighborhood, $U$, of the origin in $\mathbf{R}^{4}$. For fixed $K, \alpha, L$, let $\tilde{\mathcal{M}}_{k+1}^{0}$ be the subset of $\tilde{\mathcal{M}}_{k+1}$ with $\lambda<K L^{\alpha}$. Let $\omega$ be a formal product of cycles $\left[\Sigma_{1}\right], \ldots,\left[\Sigma_{n}\right] \in H_{*}(X)$ such that $\operatorname{deg}(\mu(\omega))=\operatorname{dim}\left(\mathcal{M}_{k}\right)$, so that the Donaldson invariant $D(\omega)$ is computed on the $k$-th moduli space $\mathcal{M}_{k}$.

We assume that the classes $\left\{\left[\Sigma_{i}\right]\right\}$ are represented by smooth submanifolds $\left\{\Sigma_{i}\right\}$ in general position. In particular, a subset of the $\left\{\Sigma_{i}\right\}$ can intersect only if their codimensions add up to 4 or less. Pick tubular neighborhoods $\left\{\tilde{\Sigma}_{i}\right\}$ of $\left\{\Sigma_{i}\right\}$ small enough to have the same property: a subset of the $\left\{\tilde{\Sigma}_{i}\right\}$ can intersect only if the codimensions of the corresponding $\Sigma_{i}$ 's add up to 4 or less. Similarly, we assume that the $\tilde{\Sigma}_{i}$ 's do not intersect our fixed ball $\tilde{U}$. Choose a geometric representative $V_{i}$ of each $\mu\left(\left[\Sigma_{i}\right]\right)$ that depends only on the connection restricted to $\tilde{\Sigma}_{i}$. This may be done for the 1,2, and 3-dimensional cycles as in [DK], and for the 0-dimensional $\Sigma$ 's as in $[\mathrm{DK}]$ or $[\mathrm{S} 1]$. (This allows us to identify the geometric representative of $\mu([\Sigma])$ on $\mathcal{B}_{k}$ with the geometric representative of $\mu([\Sigma])$ on $\mathcal{B}_{k+1}$. In each case it is the set of connections whose restriction to $\tilde{\Sigma}$ satisfies a certain condition). Note that the codimension of $V_{i}$ in $\mathcal{B}$ is the codimension of $\Sigma_{i}$ in $N$. Let $V_{\omega}=\cap_{i} V_{i}$. $V_{\omega}$ is a geometric representative of $\mu(\omega)$. Generically, $V_{\omega}$ will intersect $\mathcal{M}_{k}$ at a finite number of points (this number, counted with sign, is the Donaldson invariant $D(\omega)$ ), and each of these points will exhibit generic behavior. In particular, for each such point $A_{0}$, we can assume that $\operatorname{Mat}\left(F_{A_{0}}(0)\right)$ has three distinct singular values.

Theorem 4.1 Fix $\tilde{U}, \omega, K>0$, and $\alpha \in(0,2)$. For generic choices of $V_{\omega}$, as described above, and for all sufficiently small $L$, the intersection number of $\tilde{\mathcal{M}}_{k+1}^{0}$ with $V_{\omega} \cap \nu_{p} \cap \nu_{q}$ is $6 D(\omega)$.

Proof: We need to show that the only way for the boundary region of $\tilde{\mathcal{M}}_{k+1}$ to intersect $V_{\omega} \cap \nu_{p} \cap \nu_{q}$ is if a bubble is pinching off near $p$ and $q$, while the background connection in $\mathcal{M}_{k}$ is contributing to $D(\omega)$. We then must demonstrate that, under these circumstances, the problem reduces to the counting problems studied in $\S \S 2-3$.

Suppose we have a small bubble centered at a point $y$ that is not in $\tilde{U}$. The point $y$ can lie in at most 4 of the $\bar{\Sigma}_{i}$ 's, with the corresponding $\Sigma_{i}$ 's having total codimension 4 or less. Recall that we are using the explicit formula (3.1), and that outside a neighborhood of $y$ the new connection is identical to the background connection. For small $\lambda$, therefore, the bubble inserted at $y$ has no effect on the connection restricted to the remaining $\tilde{\Sigma}$ 's (which we index by $j$ ). Therefore, for a connection $\left(A_{0}, \lambda, y, m\right) \in \tilde{\mathcal{M}}_{k+1}$ to lie in $\cap_{i} V_{i}$, the background connection $A_{0} \in \mathcal{M}_{k}$ must lie in $\cap_{j} V_{j}$. However, $\mathcal{M}_{k}$ has dimension 8 less than $\tilde{\mathcal{M}}_{k+1}$, while $\cap_{j} V_{j}$ has dimension at most 4 more than $\cap_{i} V_{i}$. Since the dimension of $\mathcal{M}_{k}$ is less than the codimension of $\cap_{j} V_{j}, \cap_{j} V_{j} \cap \mathcal{M}_{k}$ is generically empty. 
Next we consider the case where a small bubble is centered in $\tilde{U}$. Then $\left\{\Sigma_{j}\right\}$ is all the cycles $\Sigma$ except the two points $p$ and $q$. For small $\lambda$, on each of the $\tilde{\Sigma}_{j}$ 's the connection form is equal to the background connection $A_{0}$, which must therefore be in $\cap_{j} V_{j} \cap \mathcal{M}_{k}$. However, now the dimension of $\mathcal{M}_{k}$ and the codimension of $\cap_{j} V_{j}$ match. $\cap_{j} V_{j} \cap \mathcal{M}_{k}$ is, by our genericity assumption, a discrete set of points, whose number (counted with sign) is the Donaldson invariant $D(\omega)$. For each of these points, the singular values of $\operatorname{Mat}\left(F_{A_{0}}(0)\right)$ are distinct.

By Theorem 3.1, for each such background $A_{0}$, and for $L$ small enough, there are exactly 6 values of $(\lambda, y, m)$ such that $\left(A_{0}, \lambda, y, m\right) \in \tilde{\mathcal{M}}_{k+1}^{0}$ has reducible curvature at $p$ and $q$. Furthermore, the intersection numbers for the local problem are all +1 . Now the orientation of $\tilde{\mathcal{M}}_{k+1}^{0}$ is the same as that of $\mathcal{M}_{k} \times U \times\left(0, K L^{\alpha}\right) \times S O(3)$ [D3, $\S 3]$.

Thus the contribution of points $\left(A_{0}, \lambda, y, m\right)$ to $D([p] \cdot[q] \cdot \omega)$, for fixed $A_{0}$, is exactly 6 times the contribution of $A_{0}$ to $D(\omega)$. Summing over the finite set $\left\{A_{0}\right\}$, we get that the contribution of $\tilde{\mathcal{M}}_{k+1}$ to $D([p] \cdot[q] \cdot \omega)$ is $6 D(\omega)$. 


\section{Differential forms and the $\mu$-map: Introduction}

Theorem 1.1, restated precisely as Theorem 4.1, is one of the two major results of this paper. It quantifies the contribution of the boundary region of moduli space to the geometric representative computation of the Donaldson invariants that appear in the simple type recursion relation. The remainder of the paper is a proof of Theorem 1.2 , which quantifies the contribution of the boundary region to a differential forms calculation of the same Donaldson invariants.

In this section we construct a de Rham-theoretic version of Donaldson's $\mu$-map using Chern-Weil theory. Recall that there is a canonical $S O(3)$-bundle $\mathcal{P} \rightarrow \mathcal{B}^{*} \times N$, and that the $\mu$-map is defined by slant product with $-\frac{1}{4} p_{1}(\mathcal{P})$. Using the $L^{2}$ metric one can produce a natural connection on $\mathcal{P}$, with curvature $\mathcal{F}$; see [DK $\S \S 5.1-2]$. By the Chern-Weil formula one has

$$
-\frac{1}{4} p_{1}(\mathcal{P})=\frac{1}{8 \pi^{2}} \operatorname{tr}(\mathcal{F} \wedge \mathcal{F}) \in \Omega^{4}\left(\mathcal{B}^{*} \times N\right),
$$

where the trace comes from the two-dimensional representation of $\mathfrak{s o}(3) \cong \mathfrak{s u}(2)$. Let us write tangent vectors to $\mathcal{B}^{*} \times N$ as pairs $(\alpha, X)$ with $\alpha \in T \mathcal{B}^{*}$ and $X \in T N$, and identify $T_{A} \mathcal{B}^{*}$ with $\operatorname{ker}\left(\left(d^{A}\right)^{*}\right) \subset \Omega^{1}(A d P)$. Further, for $\alpha, \beta \in \Omega^{1}(A d P)$, define $\{\alpha, \beta\}=-\sum_{i=0}^{4}\left[\alpha_{i}, \beta_{i}\right] \in \Omega^{0}(A d P)$, where the local $A d P$-valued functions $\alpha_{i}, \beta_{i}$ are the components of $\alpha, \beta$ relative to a local orthonormal basis of $T^{*} N$. If $A$ is irreducible, then $\mathcal{F}((\alpha, 0),(\beta, 0))=-2 G_{0}^{A}\{\alpha, \beta\}$, where $G_{0}^{A}$ is the inverse of the covariant Laplacian on $\Omega^{0}(A d P)$, and hence

$$
\begin{aligned}
\left.\mu_{d}(\omega)(\alpha, \beta, \gamma, \rho)\right|_{A}= & \int_{N}\left(\iota_{(\rho, 0) \iota} \iota_{\gamma, 0)} \iota(\beta, 0) \iota(\alpha, 0) \frac{1}{8 \pi^{2}} \operatorname{tr}(\mathcal{F} \wedge \mathcal{F})\right) \omega \\
= & \frac{1}{\pi^{2}} \int_{N} \operatorname{tr}\left(G_{0}^{A}\{\alpha, \beta\} G_{0}^{A}\{\gamma, \rho\}+G_{0}^{A}\{\alpha, \gamma\} G_{0}^{A}\{\rho, \beta\}\right. \\
& \left.\quad+G_{0}^{A}\{\alpha, \rho\} G_{0}^{A}\{\beta, \gamma\}\right) \omega .
\end{aligned}
$$

For our application it is crucial to get the combinatoric factors in (5.2) correct.

If we replace $\omega$ by $\delta_{p}$, a delta-form supported at a point $p$, the resulting form on $\mathcal{B}^{*}$ is still de Rham cohomologous to a form obtained using smooth $\omega$. Henceforth we write $\mu_{d}(p):=\mu_{d}\left(\delta_{p}\right)$. For any $p \in N$, a 4 -form representing $\mu_{d}(p)$ is given by

$$
\begin{aligned}
& \mu_{d}\left(\delta_{p}\right)(\alpha, \beta, \gamma, \rho)= \\
& \left.\quad \frac{1}{\pi^{2}} \operatorname{tr}\left(G_{0}^{A}\{\alpha, \beta\} G_{0}^{A}\{\gamma, \rho\}+G_{0}^{A}\{\alpha, \gamma\} G_{0}^{A}\{\rho, \beta\}+G_{0}^{A}\{\alpha, \rho\} G_{0}^{A}\{\beta, \gamma\}\right)\right|_{p} .
\end{aligned}
$$

To make use of (5.3) we need some concrete formulas - with calculable leading terms and small remainders - for $G_{0}^{A}\{\alpha, \beta\}$. We can obtain such formulas when $A$ is a concentrated instanton with a "charge-one bubble" and $\alpha, \beta$ come from infinitesimal 
changes in the bubble parameters (center, scale, and gluing angle). Tangent vectors of this type span an "approximate tangent space" on which very strong estimates are possible. This space, its relation to the action of the quaternionic affine group on $\mathbf{R}^{4}$, and its relation to the gluing construction in [DK], are central to the proof of Theorem 1.2. In the next section, we define the approximate tangent space precisely and study these relations in detail.

\section{Group actions and the approximate tangent space.}

Let $\mathbf{H}$ denote the quaternions and $\mathbf{H}^{*}$ the nonzero quaternions. The eight-dimensional approximate tangent space we define later is obtained by an "almost-action" of $\mathbf{H}^{*} \times$ $\mathbf{H} \cong \mathbf{R}_{+} \times S U(2) \times \mathbf{R}^{4}$ on $\mathcal{B}$, induced by an almost-action on $P$ (what "almostaction" means is explained below). Essentially, we lift from $N$ to $P$ cut-off versions of translations, dilations, and "self-dual rotations" in a gauge-invariant way.

To make this more precise, let $X$ be a vector field on $N$, and $A$ a connection on $P$. The pair $(X, A)$ defines a flow on $P$ obtained by lifting $X A$-horizontally to $P$. We thereby obtain from $X$ the "canonical flow of $X$ on $\mathcal{A}$ ", with associated vector field $A \mapsto \tilde{X}:=\iota_{X} F_{A} \in \Omega^{1}(A d P) \cong T_{A} \mathcal{A}$ (see [GP1] Proposition 4.3). The canonical flow is invariant under the gauge group, hence descends to $\mathcal{B}$. Moreover any two lifts to $P$ of a diffeomorphism of $N$ differ by a gauge transformation, and hence given an action on $N$ by any connected Lie group $G$ on $N$, the canonical flow integrates to a well-defined action of $G$ on $\mathcal{B}$, though in general not on $\mathcal{A}$. Of interest to us later will be the comparison of the canonical lift to that obtained by lifting $X$ horizontally with respect to a reference connection $A_{0}$. In that case the difference between the two tangent vectors in $T_{A} \mathcal{A}$ induced by the two flows is $d^{A} u$, where $u=\iota_{X}\left(A-A_{0}\right)$.

Now let $G$ be a Lie group acting from the left on $N$. Suppose that for each $g \in G$, the action $\Phi_{g}$ of $g$ on $N$ lifts to a bundle map $\tilde{\Phi}_{g}: P \rightarrow P$; if $G$ is connected we can obtain such lifts by using the canonical flow. (We do not require the lifts to piece together to a $G$-action.) For later purposes we will need to calculate the differential of the induced action of $G$ on $\mathcal{B}$, at any $g \in G$. This is not difficult, but it is easy to confuse the roles of $g$ and $g^{-1}$ in this calculation, and this mistake would be fatal for our application.

For each connection $A \in \mathcal{A}$, let $\Theta_{A} \in \Omega^{1}(P, \mathfrak{s u}(2))$ denote the connection form of $A$. Given a lift $\tilde{\phi}_{g}$ as above, define $g \cdot A$ to be the connection with connection form $\left(\tilde{\Phi}_{g}^{-1}\right)^{*} \Theta_{A}$. If the lifts piece together into an action of $G$ on $P$ (necessarily a left

action), then $(g, A) \rightarrow g \cdot A$ defines a left action of $G$ on $\mathcal{A}$. $\tilde{\Phi}_{g_{1} g_{2}}$ and $\tilde{\Phi}_{g_{1}} \circ \tilde{\Phi}_{g_{2}}$ are gauge-equivalent, since both are lifts of $\Phi_{g_{1} g_{2}}$, so an element-wise liftable $G$-action on $N$ always induces a $G$-action on $\mathcal{B}$, whether or not it induces one on $P$.

Now fix $\left[A_{0}\right] \in \mathcal{B}$ and define $\bar{\rho}: G \rightarrow \mathcal{B}$ by $\overline{\rho(g)}=\left[g \cdot A_{0}\right]$. This is well-defined and 
is independent of the choice of lifts. On a small enough neighborhood $U$ of any $g \in G$ we can always choose the $\tilde{\Phi}_{h}$ to vary smoothly with $h$, so that on $U$ the map $\bar{\rho}$ factors through a smooth map $\rho: G \rightarrow \mathcal{A}$ defined by $\rho(g)=g \cdot A_{0}$. Let $v=\left.\frac{d}{d t} g_{t}\right|_{t=0} \in T_{g} G$ and write $v=R_{g_{*}} w$, where $w \in T_{e} G=\mathfrak{g}$. Then

$$
\rho_{* g} v=\left.\frac{d}{d t}\left((\exp (t w) g) \cdot A_{0}\right)\right|_{t=0} .
$$

But $\tilde{\Phi}_{\exp (t w) g}=\gamma(t) \circ \tilde{\Phi}_{\exp (t w)} \circ \tilde{\Phi}_{g}$ for some gauge transformation $\gamma(t)$ varying smoothly in $t$, and hence $\left.(\exp (t w) g) \cdot A_{0}=\left((\exp (t w)) \cdot g \cdot A_{0}\right) \cdot \gamma(t)\right)$. Thus

$$
\begin{aligned}
\rho_{* g} v & =\left.\frac{d}{d t}\left(\exp (t w) \cdot g \cdot A_{0}\right)\right|_{t=0} \bmod \operatorname{Im}\left(d_{g \cdot A_{0}}\right) \\
& =\frac{d}{d t}\left(\left.\left(\tilde{\Phi}_{\exp (t w)}^{-1}\right)^{*} \omega_{g \cdot A_{0}}\right|_{t=0} \bmod \operatorname{Im}\left(d_{g \cdot A_{0}}\right) .\right.
\end{aligned}
$$

Let $\hat{w} \in \Gamma(T N)$ and $\hat{w}_{P} \in \Gamma(T P)$ be the vector fields on $N$ and $P$ induced by the infinitesimal action of $w$. Then

$$
\frac{d}{d t}\left(\left.\left(\tilde{\Phi}_{\exp (t w)}^{-1}\right)^{*} \omega_{g \cdot A_{0}}\right|_{t=0}=-\mathcal{L}_{\hat{w}_{P}} \omega_{g \cdot A_{0}}=-\iota_{\hat{w}} F_{g \cdot A_{0}} \bmod \operatorname{Im}\left(d_{g \cdot A_{0}}\right)\right.
$$

(if $\tilde{\Phi}$ is defined by the canonical flow then the " $\bmod \operatorname{Im}\left(d_{g \cdot A_{0}}\right)$ " can be erased in this line). Note that $v$ directly defines a vector field on $N$ by $\left.\hat{v}\right|_{\Phi_{g}(x)}=\left.\frac{d}{d t}\left(\Phi_{g_{t}}(x)\right)\right|_{t=0}$. Since we can take $g_{t}=\exp (t w) g$, it immediately follows that $\left.\hat{v}\right|_{\Phi_{g}(x)}=\left.\hat{w}\right|_{\Phi_{g}(x)}$ for all $x \in N$, so the vector fields $\hat{v}$ and $\hat{w}$ are the same. Hence

$$
\rho_{* g} v=-\iota_{\hat{v}} F_{g \cdot A_{0}} \bmod \operatorname{Im}\left(d_{g \cdot A_{0}}\right) .
$$

Thus if we identify $T_{[A]} \mathcal{B}$ with $\operatorname{ker}\left(\left(d^{A}\right)^{*}\right) \subset \Omega_{1}(A d P)$, then

$$
\bar{\rho}_{* g} X=-\pi_{A}^{\prime} \iota_{\hat{X}} F_{g \cdot A_{0}} .
$$

where $\pi_{A}^{\prime}: \Omega^{1}(A d P) \rightarrow \operatorname{ker}\left(\left(d^{A}\right)^{*}\right)$ is the $L^{2}$-orthogonal projection. (Here and below, for notational convenience we do not distinguish between a tangent vector to $\mathcal{B}$ of $\mathcal{M}$ at $[A]$, literally a gauge-invariant section of a certain vector bundle over the gaugeorbit through $A$, and the representative of this section at $A$.)

We would like to apply these ideas to the situation of a local action of $\mathbf{H}^{*} \times \mathbf{H}$ on a neighborhood of a point in $N$. Given a concentrated connection $A$, with scale $\lambda=$ $\lambda(A)$ and center point $p_{A}$, fix a positively oriented normal coordinate system centered at $p_{A}{ }^{1}$ Near $p_{A}$ it makes sense to speak of the translation, dilation, and rotation

\footnotetext{
${ }^{1}$ The precise definitions of $\lambda$ and $p_{A}$ are not important here. There are several definitions in the literature, leading to some arbitrariness in the definition of "near", "bubble", etc. In all instances in which the differences among these definitions have been carefully analyzed, it has been found that these differences do not affect the estimates we need in any material way (cf. [GP2], section 5). We will simply assume in this paper that the same is true here, and will freely quote results proved using different definitions as if they had been proved using consistent definitions of scale and center.
} 
vector fields. These are determined invariantly by data (b, $a, \alpha) \in T_{p_{A}} M \oplus \mathbf{R} \oplus \Lambda^{2}(T N)$ by setting

$$
\hat{X}_{(\mathbf{b}, a, \alpha)}=b^{j} \frac{\partial}{\partial x^{j}}+\left(\sqrt{2} \lambda^{-1}\right)\left(a x^{i} \frac{\partial}{\partial x^{i}}+\alpha_{i j} x^{i} \frac{\partial}{\partial x^{j}}\right)
$$

where $\left\{x^{i}\right\}$ are normal coordinates centered at $p_{A}$ and $b^{j}, \alpha_{i j}$ are the associated components of $\mathbf{b}, \alpha$. We include the normalization factor $(\sqrt{2} \lambda)^{-1}$ to arrange $\left\|\iota_{\hat{X}} F_{A}\right\|_{2} \approx$ const (independent of $\lambda$ ); see Proposition 6.4 below. We call $\alpha_{i j} x^{i} \frac{\partial}{\partial x^{j}}$ a self-dual/antiself-dual rotation vector field if $\alpha_{i j} d x^{i} \wedge d x^{j}$ is a SD/ASD two-form at $p_{A}$.

Since the $\hat{X}$ are only defined locally, we extend them to $N$ by cutting them off outside a small ball. For this purpose $\hat{X}$ as above we define $X=\beta \hat{X}$, where $\beta$ is a cutoff of scale

$$
\epsilon=4 n_{0} \lambda^{1 / 2}
$$

Here $n_{0}$ is a constant taken large enough to ensure that $\beta$ can be used in the gluing constructions of $[\mathrm{DK}]$, but for all of our other applications we can ignore $n_{0}$. For convenience we take $\beta=\beta_{\text {std }}\left(r_{A} / \epsilon\right)$, where $r_{A}$ is distance to $p_{A}$ and $\beta_{\text {std }}$ is a cutoff function with support in $[0,2]$, identically 1 on $[0,1]$. (These cutoffs, which will be used for the rest of this paper, are different from the ones in $\S 3$.)

We define

$$
\mathfrak{h}_{A}=\left\{X_{(\mathbf{b}, a, \alpha)}=\beta \hat{X}_{(\mathbf{b}, a, \alpha)} \in \Gamma(T N) \mid(\mathbf{b}, a, \alpha) \in T_{p_{A}} M \oplus \mathbf{R} \oplus \Lambda_{+}^{2}(T N)\right\}
$$

It is worthwhile to observe that the definition of (A)SD rotation vector fields is necessarily a local definition, since globally a nontrivial exact 2-form cannot be SD or ASD on an orientable compact manifold. In fact on $S^{4}$, rotations that are SD at one pole are ASD at the other. This is most easily seen by using stereographic projection to identify $S^{4}-\{\infty\}$ with $\mathbf{R}^{4}$, then with $\mathbf{H}$. Left-multiplication by unit quaternions induces SD rotations near 0, while right-multiplication induces ASD rotations near 0. But coordinates near $\infty$ on $S^{4}$ are related to those near 0 by quaternionic inversion (the orientation-preserving map $x \mapsto x^{-1}$ ), which interchanges the roles of left- and right-multiplication.

When $A$ is ASD, we make the following definition.

Definition 6.1 The approximate tangent space $\mathcal{H}_{A}$ at $A$ to the moduli space is the space

$$
\left\{\tilde{X}_{A}:=\iota_{X} F_{A} \mid X \in \mathfrak{h}_{A}\right\}
$$

We usually write simply $\tilde{X}$ and leave the $A$-dependence implicit.

To justify this terminology, we consider the action induced by such $X$ on an ASD connection. Since the $X$ 's are nearly conformal vector fields, one expects the induced flow to map an ASD connection to a nearly ASD connection. Proposition 6.4 below shows that this is the case, and more-but first we need a definition and a lemma. 
Definition 6.2 Given $\kappa, \nu, \lambda_{0}>0$, let $\mathcal{M}_{k+1, \lambda_{0}}^{\kappa, \nu} \subset \mathcal{M}_{k+1, \lambda_{0}}^{\prime}$ denote the subset of instantons $[A]$ obeying the conditions

(i) the first eigenvalues of the Laplacians $\left(d^{A}\right)^{*} d^{A}$ on zero-forms, $d_{+}^{A}\left(d_{+}^{A}\right)^{*}$ on $\mathrm{SD}$ two-forms, are both greater than $\nu$,

and

(ii) for all $p \in N$,

$$
\left|F_{A}(p)\right| \leq C \lambda^{2} /\left(\lambda^{2}+r_{A}(p)^{2}\right)^{2}+\kappa
$$

where $F_{A}$ is the curvature of $A, r_{A}(p)=\operatorname{dist}\left(p_{A}, p\right)$, and $\lambda, p_{A}$ are the scale and center point of $A$, respectively.

The pointwise bound (6.10) essentially says that $\left|F_{A}\right|$ is bounded by the curvature of a standard instanton plus a contribution $\kappa$ from a background connection. At small distances from $p_{A}$, the latter term is negligible, but far from $p_{A}$ the background term dominates.

When dealing with estimates for the approximate tangent space, one must decide at what scale $\epsilon$ to cut off the vector fields in $\mathfrak{h}_{A}$. If one takes $\epsilon$ too small, the derivatives of the cutoff function become inconveniently large, while if one takes $\epsilon$ too large, the contribution from the background connection swamps the contribution from the concentrated curvature. If we require that $\epsilon$ scale as a power of $\lambda$, we get the optimal balance between these undesirable features only if $\epsilon \sim \lambda^{1 / 2}$. Earlier we chose $\epsilon=4 n_{0} \lambda^{1 / 2}$, and we now take $n_{0}$ large enough so that in the gluing construction of $[\mathrm{DK}]$ one is assured of landing in the domain of Taubes' contracting mapping argument. Once $n_{0}$ has been so chosen, it (like $\kappa$ ) is simply another ignorable constant for the computations we need in this section. In particular, note that on the ball $B\left(p_{A}, \epsilon\right)$ or radius $\epsilon$ centered at $p_{A}$, we have $\lambda^{2} /\left(\lambda^{2}+r_{A}^{2}\right)^{2} \geq\left(\lambda_{0}+64 n_{0}^{2}\right)^{-2} \geq$ const $\cdot \kappa$. Hence, with a new constant $c=c(\kappa)$,

$$
\left|F_{A}\right| \leq c \lambda^{2} /\left(\lambda^{2}+r_{A}^{2}\right)^{2} \quad \text { on } \operatorname{supp}(\beta) .
$$

This enormously simplifies our computations.

The next lemma shows that we can always arrange the fiber $Z$ to lie in some $\mathcal{M}_{k+1, \lambda_{0}}^{\kappa, \nu}$

Lemma 6.3 Given small $\lambda_{0}$, let $T: \mathcal{M}_{k+1, \lambda_{0}}^{\prime} \rightarrow \mathcal{M}_{k}$ be the projection sending a concentrated connection to a "background" connection. Let $\left[A_{0}\right] \in \mathcal{M}_{k}^{\prime}$ and assume that the first eigenvalues of the Laplacians $d_{A_{0}}^{*} d_{A_{0}}, d_{A_{0}}^{+}\left(d_{A_{0}}^{+}\right)^{*}$ on Ad P-valued 0 -forms and $S D$ 2-forms, respectively, are positive. Then there exist $\lambda_{0}>0, \nu>0, \kappa>0, C>$ 0 , and a neighborhood $\mathcal{U}$ of $\left[A_{0}\right]$ in $\mathcal{M}_{k+1}$ such that $T^{-1}(\mathcal{U}) \subset \mathcal{M}_{k+1, \lambda_{0}}^{\kappa, \nu}$. 
Proof: That condition (i) in the definition of $\mathcal{M}_{k+1, \lambda_{0}}^{\kappa, \nu}$ can be satisfied follows from the proof of Lemma 7.1.24 in [DK]; that condition (ii) can be satisfied follows from modifying several ideas in [GP2] (Definition 4.1, Lemma 4.3d, and Propositions 4.4).

Henceforth we will always assume that instantons $[A]$ lie in a fixed $\mathcal{M}^{\kappa, \nu}$. For such connections we have the following.

Proposition 6.4 Fix $\kappa, \nu$. Let $\pi_{A}: \Omega^{1}(A d P) \rightarrow H_{A}^{1}:=\operatorname{ker}\left(\left(d^{A}\right)^{*}\right) \bigcap \operatorname{ker}\left(d_{+}^{A}\right)$ (naturally identified with the tangent space $\left.T_{[A]} \mathcal{M}\right)$ be the $L^{2}$-orthogonal projection. For all sufficiently small, positive $\delta$ there exist $c, \epsilon_{1}(\lambda)$, such that if $A \in \mathcal{M}_{k+1, \lambda_{0}}^{\kappa, \nu}$ then

$$
\left|\left\|\tilde{X}_{(\mathbf{b}, a, \alpha)}\right\|_{2}^{2}-8 \pi^{2}\left(|\mathbf{b}|^{2}+|a|^{2}+|\alpha|^{2}\right)\right| \leq \epsilon_{1}(\lambda)\left(|\mathbf{b}|^{2}+|a|^{2}+|\alpha|^{2}\right)
$$

where $\epsilon_{1}(\lambda) \rightarrow 0$ as $\lambda \rightarrow 0$, and

$$
\left\|\tilde{X}-\pi_{A} \tilde{X}\right\|_{2} \leq c \lambda^{\delta}\left(|\mathbf{b}| \lambda+(|a|+|\alpha|) \lambda^{1 / 2}\right) .
$$

Proof: The proof of the first statement is similar to that of [GP1, Proposition 3.6]; we omit the details. We prove the second statement later as Proposition 10.8(b).

Thus by taking $\lambda$ small enough we can ensure that $\pi_{A}: \mathcal{H}_{A} \rightarrow H_{A}^{1}$ is injective. Let $O_{0} \subset \mathfrak{h}_{A}$ be an open neighborhood of zero. For $X \in \mathfrak{h}_{A}$, let $A^{X}$ denote the connection that results from acting on $A$ by the canonical flow of $X$ for unit time, and let $O_{A}=\left\{A^{X} \mid X \in O_{0}\right\}$. Proposition 6.4 has two implications, once we take $O_{0}$ small enough. First, $O_{A}$ lies in a neighborhood of the ASD connections on which Taubes' contracting-mapping argument lets us "project" the image of $A$ to an ASD connection. Second, by the implicit function theorem, the image of $O_{A}$ in $\mathcal{M}_{k+1}$ is an 8-dimensional submanifold of $\mathcal{M}_{k+1}$.

The quantity $\tilde{X}-\pi_{A} \tilde{X}$ will be central to the definition of the remainder terms in $\mu_{\text {loc }}(p)$ and to the analysis in $\S 10$. We define

$$
\begin{aligned}
\xi_{X} & =\tilde{X}-\pi_{A} \tilde{X} \\
& =d^{A} G_{0}^{A}\left(d^{A}\right)^{*} \tilde{X}+\left(d_{+}^{A}\right)^{*} G_{+}^{A} d_{+}^{A} \tilde{X}
\end{aligned}
$$

Here $G_{0}^{A}$ and $G_{+}^{A}$ are the inverses of the Laplacians $\left(d^{A}\right)^{*} d^{A}$ and $d^{A}\left(d_{+}^{A}\right)^{*}$ on $\Omega^{0}(A d P)$ and $\Omega_{+}^{2}(A d P)$, respectively. 
We make three observations here. First, if $A$ is $\mathrm{ASD},\left\|\left(d_{+}^{A}\right)^{*} G_{+}^{A} d_{+}^{A} \tilde{X}\right\|_{2} /\|\tilde{X}\|_{2}$ is small for any rotation vector field, not just SD ones. This is to be expected since any rotation vector field is an approximate isometry and hence should approximately preserve anti-self-duality. However $\left\|d^{A} G_{0}^{A}\left(d^{A}\right)^{*} \tilde{X}\right\|_{2} /\|\tilde{X}\|_{2}$ is small only for the rotation vector fields of duality opposite to that of the connection. Second, to deduce from this smallness that the parameter space injects (locally) into $\mathcal{B}$, one must know that the first eigenvalue of the Laplacians on zero-forms does not tend to 0 as $\lambda$ tends to zero, as it will if the "background" connection is flat (or merely reducible). Indeed on $\mathcal{M}_{1}\left(S^{4}\right)$, all rotation vector fields (not cut off), lifted as above, preserve the standard instanton. (On $\mathcal{M}_{1}\left(\mathbf{R}^{4}\right)$, if one writes instantons in the usual gauge and instead lifts rotations using the flat connection, then ASD rotations preserve the standard ASD instantons centered at the origin, while SD vector fields induce the effect of a gauge transformation.) Third, because of the cutoff $\beta, \mathfrak{h}_{A}$ is not a Lie subalgebra of $\Gamma(T N)$, although in some sense it is close to being one. Thus, while intuitively $\mathfrak{h}_{A}$ is associated with the Lie algebra of an 8-dimensional group of translations, dilations, and rotations, $O_{A}$ is not quite the orbit of an 8-dimensional local Lie group, hence the term "almost-action".

We will return to this point at the end of this section, but first we wish to relate $O_{A}$ to the gluing construction in [DK]. The fibration of a region in $\mathcal{M}_{k+1, \lambda_{0}}^{\prime}$ over $\mathcal{M}_{k}^{\prime}$ is usually viewed in terms of center point, scale, and gluing parameter. We claim that on an infinitesimal level these are essentially the eight parameters used to define the approximate tangent space. Indeed [GP2, §5] it was shown that lifts using the translation and dilation vector fields do correspond to infinitesimal changes in center point and scale, up to an error that is essentially $O(\lambda)$. ([GP2] dealt only with $\mathcal{M}_{1}$, but under a suitable definition of "concentrated" the same argument works more generally). It remains to identify our action of $S O(3)$ (the SD rotations) with the "gluing parameters" of the construction in [DK, §7.2]. As both constructions are non-canonical we content ourselves with a somewhat heuristic correspondence.

Instantons in the subspace $\mathcal{M}_{k+1, \lambda_{0}}^{\kappa, \nu}$ have a single "charge-one bubble" and are otherwise not concentrated. For any such ASD reference connection $A_{0}=A$ there exists a gauge over ball $B\left(p_{A}, K \lambda\right)$ such that after pulling back to $B(0, K \lambda) \subset \mathbf{R}^{4}$ by a positively-oriented normal coordinate system $\left\{x^{i}\right\}$, the connection form is close to $A_{\lambda}^{\text {std }}$, the standard instanton on $\mathbf{R}^{4}$ of scale $\lambda$ and center the origin. (See [DK], §8.2.1.) Here $K>1$ is any fixed number and "close" means that after dilating by $\lambda$, the two connections are $C^{2}$-close on $B(0, K) \subset \mathbf{R}^{4}$; the undilated connections satisfy $\mid\left(\nabla^{j}\left(A-A_{\lambda}^{\text {std }}\right) \mid \leq \epsilon_{1} \lambda^{-1-j}, 0 \leq j \leq 2\right.$, where by taking $\lambda_{0}$ small enough we can take $\epsilon_{1}$ as small as we please. After a choice of normal coordinate system, the identification $\mathbf{R}^{4} \cong \mathbf{H}$, and an identification of $S U(2)$ with the unit quaternions, the connection form for $A_{\lambda}^{\text {std }}$ on our ball is

$$
\omega_{0}=\frac{\operatorname{Im}(\bar{x} d x)}{\lambda^{2}+|x|^{2}} .
$$


For integers $j=1, \ldots, 10$ define the sets $U_{j}=B\left(p_{A}, j n_{0} \lambda^{1 / 2}\right)$ and $V_{j}=N-U_{j}$. Also let let $U_{\infty}$ denote the annulus $U_{10} \cap V_{1}$ and let $\Omega$ denote the smaller annulus $U_{9} \cap V_{2}$. We choose gauges $s_{0}, s_{\infty}$ (local sections of $P$ ) over $U_{10}, U_{\infty}$ respectively such that the transition function between $U_{10}$ and $U_{\infty}$ is $g_{0 \infty}(x):=\bar{x} /|x|$ (i.e. $s_{\infty}=s_{0} g_{0 \infty}$ ); furthermore we take $s_{0}$ to be the radial gauge for $A$ with respect to $p_{A}$ in which (6.15) is written. The connection form for $A_{\lambda}^{\text {std }}$ with respect to $s_{\infty}$ on $U_{\infty}$ is then

$$
\omega_{\infty}=\frac{\lambda^{2} \operatorname{Im}(x \overline{d x})}{|x|^{2}\left(\lambda^{2}+|x|^{2}\right)} .
$$

To make contact with the construction in $[\mathrm{DK}]$, we will pretend that on the ball $U_{8}$, our connection $A$ is exactly standard (so that the connection form relative to $s_{0}$ on $U_{8}$ is (6.15)). Let $\tilde{\beta}$ be a function that is identically 1 on $N-U_{\infty}$ and identically 0 on $\Omega$, with $|\nabla \tilde{\beta}| \leq c n_{0}^{-1} \lambda^{-1 / 2}$. (Note that the "interior" part of the support of $\tilde{\beta}$ occurs where $\beta \equiv 1$.) On $U_{\infty}$ let $\omega_{\infty}^{\prime}$ denote the connection form $\tilde{\beta} \omega^{\prime}$. We then define a new connection $A^{\prime}$ on $P$ by declaring the connection form for $A^{\prime}$ in the gauge $s_{\infty}$ over $U_{\infty}$ to be $\omega_{\infty}^{\prime}$, and declaring $A^{\prime}=A$ on $N-U_{\infty}$. We think of $\left.A^{\prime}\right|_{V_{8}}$ as a cut-off "background connection". In fact, we can define a bundle $P_{k}$ of Pontryagin index $k$ by replacing the transition function $g_{0 \infty}$ by the identity; $\left.A^{\prime}\right|_{V_{8}}$ extends to a connection on $P_{k}$ that is flat near $p_{A}$. Note that over $\Omega$ the connection $A^{\prime}$ is flat; its connection form there, relative to $s_{0}$, is $g_{0 \infty} d g_{0 \infty}^{-1}=\operatorname{Im}(\bar{x} d x) /|x|^{2}$.

Our choice of normal coordinates and identification $\mathbf{R}^{4} \cong \mathbf{H}$ induces a Lie algebra isomorphism $\theta: \Lambda_{+}^{2} T_{p_{A}}^{*} M \rightarrow \mathfrak{s u}(2)=\operatorname{Im}(\mathbf{H})$, mapping the standard basis of $\Lambda_{+}^{2} T_{0}^{*} \mathbf{R}^{4}$ to $\{\mathbf{i}, \mathbf{j}, \mathbf{k}\}$. (Alternatively, $\theta^{-1}$ is given by mapping $v \in \mathfrak{s u}(2)$ first to the vector field induced by quaternionic left-multiplication on $\mathbf{H}$, then to the two-form obtained by lowering an index using the metric.) Let $v=\theta(\alpha)$ and assume $|v|$ is not too large. We consider the canonical flow of $X=\beta \alpha_{i j} x^{i} \frac{\partial}{\partial x^{j}}$ acting on the cut-off connection $A^{\prime}$. After integrating the flow for time 1, the action on the base is $x \mapsto \phi(x)=h_{1}(r) x$, where $r=|x|$ and where $h_{1}(r)=\exp (\beta v)$. Let $A_{v}^{\prime}$ be the connection determined by this integrated canonical flow.

An alternative flow, the " $s_{0}$-flat" flow, is obtained by lifting $X$ to $\left.P\right|_{U_{8}}$ using the flat connection determined by $s_{0}$, and extending this flow to the complement of $U_{8}$ by the identity (since $X$ is supported in $U_{8}$ ). If we integrate the $s_{0}$-flat flow for time 1 , the resulting connection form on $\Omega$ with respect to $s_{0}$ is

$$
\omega_{v}^{\prime}=\frac{\operatorname{Im}\left(\overline{h_{1}(r) x} d\left(h_{1}(r) x\right)\right)}{|x|^{2}}=g_{0 \infty} h_{1}^{-1} d h_{1} g_{0 \infty}^{-1}+g_{0 \infty} d g_{0 \infty}^{-1} .
$$

By our earlier comments, the connections resulting from the canonical flow and the $s_{0}$-flat flow are gauge equivalent (and in fact are equal outside $U_{8}$ ). Thus $A_{v}^{\prime}$ is gaugeequivalent to a connection $A_{v}^{\prime \prime}$ equaling $A^{\prime}$ on $N-U_{\infty}$, and whose connection form in $\Omega$ (in the gauge $s_{0}$ ) is (6.17). 
We claim that the connection $A_{v}^{\prime \prime}$ is the one constructed in [DK, p. 296]. The latter essentially begins with the connection $A^{\prime}$ (thought of as a cut-off connection on $\left.P_{k}\right|_{V_{1}}$ glued to a connection on a $k=1$-bundle $\left.\left.P_{1}\right|_{U_{10}}\right)$ and modifies it on $U_{\infty}$ as follows. Let $h_{1}(r)$ be as above, let $h_{2}(r)=\exp (-(1-\beta) v)$ and consider the two gauge transformations $\tilde{h}_{1}, \tilde{h}_{2}$ over $U_{\infty}$ given by $\tilde{h}_{i}\left(s_{\infty}(x)\right)=s_{\infty}(x) h_{i}(r)$. The gauge transformation $\tilde{h}_{1}$ does not extend to all of $P$ (unless $\exp (v)= \pm 1$ ), but it does extend to the bundle $P_{k}$ defined earlier, and for $r \leq 4 n_{0} \lambda^{1 / 2}$ changes the trivialization $s_{\infty}$ (extended to $P_{k}$ ) by the constant $\exp (v)$. Similarly the gauge transformation $\tilde{h_{2}}$ extends to $\left.P\right|_{U_{10}}$, changing the trivialization $s_{\infty}$ for $r \geq 8 n_{0} \lambda^{1 / 2}$ by $\exp (-v)$. Because $h_{1}^{-1} d h_{1}=h_{2}^{-1} d h_{2}$, the two gauge transformations have the same effect on the flat connection $\left.A^{\prime}\right|_{\Omega}$. Therefore we can define a new connection $A_{v}^{\mathrm{DK}}$ by

$$
A_{v}^{\mathrm{DK}}=\left\{\begin{array}{l}
\tilde{h}_{2}\left(A^{\prime}\right) \text { on } U_{9} \\
\tilde{h}_{1}\left(A^{\prime}\right) \text { on } V_{2}
\end{array}\right.
$$

The connection form for $A_{v}^{\mathrm{DK}}$ with respect to $s_{\infty}$ on $\Omega$ is $h_{1}^{-1} d h_{1}=h_{2}^{-1} d h_{2}$, so with respect to $s_{0}$ the connection form is precisely (6.17). Thus $A_{v}^{\prime \prime}$ and $A_{v}^{\mathrm{DK}}$ coincide on $\Omega$. Since $\tilde{h}_{1} \equiv 1$ on $V_{9}$ we have $A_{v}^{\mathrm{DK}}=A^{\prime}$ on this region, and since $X \equiv 0$ on $V_{9}$ we have $A_{v}^{\prime \prime}=A^{\prime}$ there as well. Thus $A_{v}^{\prime \prime}=A^{\mathrm{DK}}$ on $V_{2}$. It remains to consider only $U_{2}$. On this ball, a computation shows that the connection form for $A^{\prime}$ relative to $s_{0}$ is

$$
\omega_{0}^{\prime}=\frac{\operatorname{Im}(\bar{x} d x)}{|x|^{2}}\left(1-\tilde{\beta}(r) \frac{\lambda^{2}}{\lambda^{2}+|x|^{2}}\right) .
$$

On $U_{2}$, we have $h_{2} \equiv 1$, so the connection form for $A_{v}^{\mathrm{DK}}$ remains $\omega_{0}^{\prime}$. But the connection $A^{\prime}$ is also preserved by the " $s_{0}$-flat" flow of $X$; replacing $x$ by $h_{1}(r) x$ in $(6.19)$ does not change $\omega_{0}$, since $h_{1}$ is constant on $U_{2}$. Therefore $A_{v}^{\prime \prime}=A_{v}^{\mathrm{DK}}$ over all of $N$.

Now let $A_{v}$ be the connection obtained from applying the canonical flow of $X$ for time 1 to $A$ (rather than to $A^{\prime}$ ). The preceding shows that up to gauge equivalence, when $|v|$ is not too large the only differences between $A_{v}$ and $A_{v}^{\mathrm{DK}}$ arise from the facts that (i) $A$ is only approximately standard on a small ball $B\left(p_{A}, K \lambda\right)$ rather than exactly standard on the larger ball $B\left(p_{A}, 10 n_{0} \lambda^{1 / 2}\right)$, and (ii) we do not cut off $A_{v}$ before applying the flow.

It should also be noted that since the subspace $\mathfrak{h}_{A}^{\text {rot }}$ corresponding to the SD rotation vector fields is not closed under Lie bracket, if we let $\mathfrak{h}_{A}^{\text {rot }}$ act on $A$ by the canonical flow for time 1, we should not expect to get a closed "orbit". But the construction in $[\mathrm{DK}]$ shows that the space of gluing parameters is a copy of $S O(3)$.

To address this discrepancy, first note that if $N$ were $\mathbf{R}^{4}$ we could dispense with the cutoffs in the definition of $\mathfrak{h}_{A}$. The vector fields would be globally defined, and would generate a Lie algebra exponentiating to the group of motions of $\mathbf{R}^{4}$,

$$
\left\{x \mapsto a x+b \mid(a, b) \in \mathbf{H}^{*} \times \mathbf{H}\right\} .
$$


The stabilizer of the origin would be $\mathbf{H}^{*} \times\{0\}$, and if the initial connection $A$ were standard, the set of connections generated by letting $S U(2) \subset \mathbf{H}^{*}$ act via the canonical flow would give two copies of the space obtained by the construction in [DK], as $(a, 0)$ and $(-a, 0)$ yield the same connection. (Alternatively, if $v \neq 0$ is small enough, the connections $A^{\prime}=A^{\mathrm{DK}}$ and $A_{v}^{\prime \prime}=A_{v}^{\mathrm{DK}}$ are gauge-inequivalent, because the gauge transformation $\tilde{h}_{1}$ defined earlier - which always extends to $\left.P\right|_{N-\left\{p_{A}\right\}}$-extends to $P$ if and only if $\exp (v)= \pm 1$.) From our earlier discussion the action of $(a, b)$ on the standard instanton is given by pulling back the connection form by the inverse of $(a, b)$, which results in a connection of scale $|a|$ and center $b$ (cf. (8.23)). The unit quaternion $a /|a|$ corresponds to gluing angle, doubly parametrized.

Intuitively, then, we have the following picture. Fix a reference connection $A=$ $A_{0} \in \mathcal{M}_{k+1, \lambda_{0}}^{\kappa, \nu}$. Let $B \subset \mathfrak{s u}(2)$ be the ball centered at the origin that is carried diffeomorphically to $S U(2)-\{-1\}$ by the exponential map, and let $B^{\prime} \subset \mathfrak{h}_{A}^{\text {rot }}$ be the corresponding set of SD rotation vector fields. If we let the canonical flow of elements in $B^{\prime}$ act for time 1 on $\left[A_{0}\right]$ we obtain a space that (for purposes of integrating reasonably behaved differential forms) approximates two copies of the fiber $Z_{\mathrm{DK}}$. This correspondence becomes sharper as $\lambda_{0} \rightarrow 0$ : as we take the limit and rescale the (local) metric and normal coordinates correspondingly, the failure of $\mathfrak{h}_{A}$ to close under Lie bracket disappears on any ball of fixed rescaled size. Furthermore, because the rescaled metric becomes flat, the limiting space of vector fields $\mathfrak{h}_{A}$ is the same whether we center the rotations and dilations at $p_{A}$ or at $p$. Thus the limiting action of $\mathbf{H}^{*} \times \mathbf{H}$ above appears to generate an immersed manifold that we can treat "homologically" as two copies of $Z_{\mathrm{DK}}$. This discussion motivates the assumptions we make on $Z$ in the next section.

\section{The fiber $\mathrm{Z}$.}

For our purposes we need only consider one fiber $Z=Z_{\lambda_{0}}$ of the projection $\mathcal{M}_{k+1, \lambda_{0}}^{\prime} \rightarrow$ $\mathcal{M}_{k}^{\prime}$; we do not need to construct the whole fibration. We will assume $Z$ has the following five properties. The first three are known to be satisfied by $Z_{\mathrm{DK}}$, so the key assumptions are really the last two, which require the tangent spaces of $Z$ and $Z_{\mathrm{DK}}$ to be close in various norms. The assumptions can almost certainly be weakened from those below, at the cost of considerably more technical work.

(Z1) $Z$ fibers over $N$ via the projection $Z \rightarrow N$ sending a concentrated connection to its center. Given $U \subset N$ we let $\left.Z\right|_{U}$ denote the inverse image of $U$ under the projection. We assume that $N$ can be covered by a finite number of normal coordinate charts $U_{i}$ (which we may take to be geodesic balls) such that for each $i$ there is a two-to-one fiber-preserving covering map $\bar{\rho}_{i}: \mathbf{H}_{\lambda_{0}}^{*} \times\left. U_{i} \rightarrow Z\right|_{U_{i}}$ having additional properties listed below. Here $\mathbf{H}_{\lambda_{0}}^{*}=\left\{a \in \mathbf{H}^{*}|| a \mid<\lambda_{0}\right\} \cong\left(0, \lambda_{0}\right) \times S U(2)$, where the isomorphism is $a \mapsto(|a|, a /|a|)$. (Note that the center-point and scale maps are 
defined globally on $Z$; it is only for the purpose of handling gluing parameters that we need to chop up $N$.)

In general a normal coordinate system $\left\{x^{j}\right\}$ on $U_{i}$ determines an identification between $U_{i}$ and a ball in $\mathbf{H}$ centered at the origin, and hence a local action of $\mathbf{H}_{\lambda_{0}}^{*} \times \mathbf{H}$ on $U_{i}$ given by $((a, b), x) \mapsto a x+b$. We assume that on each $U_{i}$ there is a positively oriented normal coordinate system $\left\{x^{j}\right\}$ on $U_{i}$ such that $\bar{\rho}_{i}$ is approximately given by the induced canonical flow of this $\mathbf{H}_{\lambda_{0}}^{*} \times \mathbf{H}$-action, based at the standard instanton on $\mathbf{R}^{4}$, in the sense that $(\mathbf{Z 2})-(\mathbf{Z 5})$ below are true. From $[\mathrm{D}, \S 3]$, the orientation induced on the fiber $Z$ by the standard orientation of $\mathbf{H} \times \mathbf{H}$ as a complex vector space is then compatible with the standard orientations of $\mathcal{M}_{k+1}$ and $\mathcal{M}_{k}$ (i.e. the orientation of $\mathcal{M}_{k+1, \lambda_{0}}^{\prime}$ is the product of the orientation of $Z$ and the pullback of the orientation of $\left.\mathcal{M}_{k}\right)$. These are the orientations used in (1.8).

(Z2) We assume that for each $i$, the scale and center point of $A=\bar{\rho}_{i}(a, b)$ are $\lambda(A)=|a|$ and $p_{A}=b$ (in quaternionic normal coordinates) respectively.

(Z3) Given $i$, let $\left[A_{a, b}\right]=\bar{\rho}_{i}(a, b)$ and let $F_{a, b}=F_{A_{a, b}}$. We assume that for any $K>0$, on the ball $B\left(p_{A}, K \lambda(A)\right)$ we have

$$
|| F_{a, b}\left|-\frac{\sqrt{48}|a|^{2}}{\left(|a|^{2}+|x-b|^{2}\right)^{2}}\right| \leq \epsilon_{1}(\lambda) \lambda^{-2}
$$

where $\epsilon_{1}(\lambda) \rightarrow 0$ as $\lambda \rightarrow 0$.

(Z4) Let $B$ be the component of $\exp ^{-1}(S U(2)-\{-1\})$ containing 0 . A tangent vector $v \in T_{(a, b)}\left(\mathbf{H}_{\lambda_{0}}^{*} \times B\right)$ gives rise to a vector field on a neighborhood of $b \in B$ that determines an element $X_{v} \in \mathfrak{h}_{A}$. Writing $\tilde{X}_{v}=\iota_{X_{v}} F_{A}$, we require that

$$
\left\|\bar{\rho}_{*} v+\tilde{X}_{v}\right\|_{L^{2}} \leq \epsilon_{2}(\lambda)|v|
$$

where $\epsilon_{2}(\lambda) \rightarrow 0$ as $\lambda \rightarrow 0$. Observe that because of (6.12), we can alternatively write (7.2) as $\left\|\bar{\rho}_{*} v-\left(-\pi_{A} \tilde{X}_{v}\right)\right\|_{L^{2}} \leq \epsilon_{2}(\lambda)|v|$; cf. (6.5).

(Z5) Letting $\xi_{v}^{\prime}=\bar{\rho}_{*} v+\tilde{X}_{v}$ and $\xi_{v}=\tilde{X}_{v}-\pi^{A} \tilde{X}_{v}$, we further require that $\xi_{v}^{\prime}$ satisfy the same weighted $L^{4}$ bounds as $\xi_{v}$ given in Proposition 10.8 ((10.74) and (10.75)), and the pointwise bound (10.72).

If not for $(\mathbf{Z 4})$ and $(\mathbf{Z 5})$, we would not need to assume $(\mathbf{Z 1})-(\mathbf{Z 3})$. By itself, $(\mathbf{Z 1})$ follows from the description of the ends of moduli space in [DK, $\S \S 7.2$ and 8.2]; we simply take the local diffeomorphism $\left(0, \lambda_{0}\right) \times S O(3) \times\left. U_{i} \cong Z\right|_{U_{i}}$, and pre-compose with the covering map $S U(2) \rightarrow S O(3)$. Similarly, $(\mathbf{Z 2})$ and (Z3) follow from [DK, $\S 8.2 .1]$.

What is not clear is whether the construction in [DK] yields a fiber whose tangent space at $[A]$ is sufficiently close to $\pi_{A}\left(\mathcal{H}_{A}\right)$ in the norms required for our analysis. If the 
subspace $\mathfrak{h}_{A} \subset \Gamma(T N)$ were a Lie subalgebra, then by (6.5) the canonical flow would generate a fiber whose tangent space at $[A]$ would be precisely $\pi_{A}\left(\mathcal{H}_{A}\right)$. However, $\mathfrak{h}_{A}$ is not closed under Lie bracket, and the canonical flow of vector fields in $\mathfrak{h}_{A_{0}}$, acting on a single reference connection $\left[A_{0}\right]$, has no chance of generating an orbit that reasonably approximates all of $Z_{[D K]}$; the cutoff in the translation vector fields prevents the canonical flow from moving the center point very far from $p_{A_{0}}$, whereas all points in $N$ can occur as center in $Z_{[D K]}$. But the estimates relevant to proving Theorem 1.2 are much less sensitive to changing the definition of of translations than to changing the definition of rotations and dilations, so it seems plausible that, by a patching argument, altering the definitions of only the translation vector fields in any significant way, we can splice together canonical flows based at connections with nearby center points. Presumably by splicing enough flows together we can obtain a fiber that is $C^{1}$-close globally to $Z_{[D K]}$ and $C^{1}$-close locally to the orbit of some canonical flow. Even if the splicing construction fails, there are two reasons why, for purposes of integration, we may not need to define a true fiber (such as $Z_{[D K]}$ ) in a topological sense. First, when we integrate $\mu(p) \wedge \mu(q)$ over an orbit of the canonical flow, only connections with center point near $p$ and $q$ contribute significantly to the integral. It is likely that the same holds for an integral over $Z_{[D K]}$, so that it suffices to approximate only a region of $Z_{[D K]}$ consisting of connections with center point in a fixed small ball. Second, although the canonical flow of the subspace $\mathfrak{h}_{A_{0}}^{\text {rot }}$ acting on $\left[A_{0}\right]$ does not generate a closed manifold, it does generate an immersed copy of $S U(2)-\{-1\}$ lying in a small neighborhood of an $S O(3)$-orbit in $Z_{[D K]}$, and which geometrically wraps twice around this orbit. A careful analysis may show that there is a homotopy from the immersed punctured $S U(2)$ to a punctured double cover of the $S O(3)$ in $Z_{[D K]}$, small enough in all relevant norms that there is only a negligible difference between integrating over $Z_{[D K]}$ and over the orbit of the canonical flow.

Thus the idea behind $(\mathbf{Z} \mathbf{1}-\mathbf{Z} \mathbf{5})$ is basically that there is fiber that interpolates between $Z_{[D K]}$ and the not-quite-fiber generated by splicing together canonical flows. The hypotheses (Z4-Z5) amount to assuming that, in this interpolated fiber, the bounds on $\xi^{\prime}$ are as good as they would be if the tangent space to the fiber were the one determined by the canonical flow. We need such an assumption because, when we pull $\mu_{d}(p) \wedge \mu_{d}(q)$ back to $Z$, we need to insert true tangent vectors to $Z$ into (5.3); the $\pi_{A} \tilde{X}$ 's in the expansion (8.1) below should be replaced by $\bar{\rho}_{*} v$ 's-which has the effect of replacing each $\xi$ in (8.6) with $\xi^{\prime}$.

There is other evidence making the simultaneous satisfaction of at least $(\mathbf{Z} \mathbf{1}-\mathbf{Z} \mathbf{4})$ very plausible. On $\mathbf{R}^{4}$, if we remove the cutoffs in the definition of $\mathfrak{h}_{A}$ and define $Z$ from the canonical flow acting on the standard instanton, then the spaces $T_{[A]} Z$ and $\mathcal{H}_{A}$ coincide. In the case of 1 -instantons over simply connected definite manifolds (where the background connection is flat and there are no gluing parameters, so $Z$ is five-dimensional), (Z4) was shown in [GP2] to be true with $\epsilon_{2}(\lambda) \leq c \lambda^{1-\delta}$ for small $\delta>0$; in [G2] this was strengthened to $\lambda^{1+\delta}$. 
The technical hypothesis (Z5) is more ad hoc, and stronger than necessary, but is not without basis. In the setting of the five-dimensional moduli spaces mentioned above, certain estimates of this paper and [G2] can be combined to show that $\left\|r_{p}^{-\delta} \xi^{\prime}\right\|_{4} \lesssim \lambda^{-1+\delta}\left(b \cdot \lambda+a \cdot \lambda^{1 / 2}\right)$ and $\left\|r_{A} \xi^{\prime}\right\|_{4} \leq b \cdot \lambda^{\delta}\left(b \cdot \lambda+a \cdot \lambda^{1 / 2}\right)$, much stronger than the $L^{p}$ bounds assumed in $(\mathbf{Z 5})$. (Here $r_{p}$ denotes distance to an arbitrary point $p \in N$.)

An important implication of $(\mathbf{Z 4})$ and (6.12) is the following. Let $d v o l_{Z}^{L^{2}}$ be the volume form on $Z$ induced by the $L^{2}$ metric on $\mathcal{B}$. (Hypothesis $(\mathbf{Z} \mathbf{1})$ determines an orientation on $Z$, so there is no sign ambiguity here.) Let $a \in \mathbf{R}^{4}$ denote the quaternionic variable in $\mathbf{H}_{\lambda_{0}}^{*}$. Then

$$
\bar{\rho}_{i}^{*}\left(d v o l_{Z}^{L^{2}}\right) \approx \mathrm{const} \cdot d^{4} a \wedge d v o l_{N}=\mathrm{const} \cdot \lambda^{3} d \lambda \wedge d v o l_{S^{3}} \wedge d v o l_{N}
$$

where the approximation becomes exact as $\lambda \rightarrow 0$ (and the constant is of course nonzero). Our chief use of (7.3) will be to help estimate the integrals of the non-local terms in $\mu_{d}(p) \wedge \mu_{d}(q)$. For this purpose, we don't actually need " $\approx$ " in $(7.3)$; " $\leq$ " would suffice. Thus hypothesis $(\mathbf{Z} 4)$ can be weakened.

\section{Localizing $\mu_{d}(p) \wedge \mu_{d}(q)$.}

From now on we assume there is a fiber $Z$ with properties $(\mathbf{Z} \mathbf{1}-\mathbf{Z} 5)$. To motivate the leading-term calculation in (1.10), suppose for the moment that for $[A] \in Z$ the tangent space $T_{[A]} Z$ is precisely, rather than approximately, the space $\pi_{A}\left(\mathcal{H}_{A}\right) \subset$ $T_{[A]} \mathcal{M}$. Then if we pull $\mu_{d}(p)$ back to $Z$, we need only apply (5.3) to arguments of the form $\pi_{A} \tilde{X}_{A}$ with $X \in \mathfrak{h}_{A}$. Recalling the definition of $\xi_{X}$ in (6.14), we then have

$$
G_{0}^{A}\left\{\pi^{A} \tilde{X}, \pi^{A} \tilde{Y}\right\}=G_{0}^{A}\left(\{\tilde{X}, \tilde{Y}\}+\operatorname{Rem}_{1}(X, Y ; A)\right)
$$

where

$$
\operatorname{Rem}_{1}(X, Y)=\left\{\tilde{X}, \xi_{Y}\right\}-\left\{\tilde{Y}, \xi_{X}\right\}+\left\{\xi_{X}, \xi_{Y}\right\} .
$$

(We omit writing most of the $A$-dependence in these formulas explicitly.) Here $\{\cdot, \cdot\}$ is a universal, local, antisymmetric bilinear pairing that takes two $A d P$-valued 1-forms and produces an $A d P$-valued zero-form. Note that $\operatorname{Rem}_{1}(X, Y)$ is antisymmetric in $X$ and $Y$.

In [G1, Proposition 2.1], it was shown how to expand several of the expressions appearing in (8.1-8.2) as a leading-order local term plus a non-local remainder, smaller in appropriate norms. In particular, for any vector fields $X, Y$ on $N$, we have

$$
G_{0}^{A}\{\tilde{X}, \tilde{Y}\}=-\frac{1}{2} F(X, Y)+G_{0}^{A}\left(R^{\prime \prime}(X, Y)\right)
$$


where $F=F_{A}$ and where

$$
\begin{aligned}
2 R^{\prime \prime}(X, Y) & =\mathcal{R}(F)(X, Y)+F(\Delta X, Y)+F(X, \Delta Y) \\
& -2\left(\nabla_{i}^{A} F\right)\left(\nabla_{i} X, Y\right)-2\left(\nabla_{i}^{A} F\right)\left(X, \nabla_{i} Y\right)-2 F\left(\nabla_{i} X, \nabla_{i} Y\right) .
\end{aligned}
$$

Here $\mathcal{R}$ is an endomorphism proportional to the Riemann tensor whose precise form does not concern us. As a consequence of $(8.3), R^{\prime \prime}(X, Y)$ is antisymmetric in $X$ and $Y$. The precise way in which the derivatives of $F$ and the derivatives of $X$ and $Y$ are hooked together in (8.4) is critical for certain estimates (Lemma 10.4).

Applying (8.3) to the first term in (8.1), we find

$$
G_{0}^{A}\{\pi \tilde{X}, \pi \tilde{Y}\}=-\frac{1}{2}\left(F(X, Y)-\operatorname{Rem}_{2}(X, Y)\right)
$$

where

$$
\begin{aligned}
\frac{1}{2} \operatorname{Rem}_{2}(X, Y) & =G_{0}^{A}\left(R^{\prime \prime}(X, Y)+\operatorname{Rem}_{1}(X, Y)\right) \\
& =G_{0}^{A}\left(R^{\prime \prime}(X, Y)+\left\{\tilde{X}, \xi_{Y}\right\}-\left\{\tilde{Y}, \xi_{X}\right\}+\left\{\xi_{X}, \xi_{Y}\right\}\right) \\
& :=G_{0}^{A}\left(\operatorname{Rem}_{2}^{\prime}(X, Y)\right) .
\end{aligned}
$$

Inserting all this into (5.3) we find

$$
\begin{aligned}
& \mu_{d}(p)(\pi \tilde{X}, \pi \tilde{Y}, \pi \tilde{V}, \pi \tilde{W})= \\
& =\frac{1}{4 \pi^{2}} \operatorname{tr}\left(\left.(F(X, Y) F(V, W)+F(X, V) F(W, Y)+F(X, W) F(Y, V))\right|_{p}\right) \\
& \quad+\left.\operatorname{Rem}_{3}(X, Y, V, W)\right|_{p}+\left.\operatorname{Rem}_{4}(X, Y, V, W)\right|_{p},
\end{aligned}
$$

where

$$
\operatorname{Rem}_{3}=\mathrm{const} \cdot \operatorname{tr}\left(F \wedge \operatorname{Rem}_{2}\right), \quad \operatorname{Rem}_{4}=\mathrm{const} \cdot \operatorname{tr}\left(\operatorname{Rem}_{2} \wedge \operatorname{Rem}_{2}\right) .
$$

(In (8.8) we regard $F$ and $R e m_{2}$ as $\Gamma(A d P)$-valued two-forms on the space of vector fields.) The first term in (8.7) is just $\left.\left(8 \pi^{2}\right)^{-1} \operatorname{tr}(F \wedge F)(X, Y, V, W)\right|_{p}$, which, since $F$ is $\mathrm{ASD}$, can be rewritten as $\left.\left(8 \pi^{2}\right)^{-1}|F|^{2} \operatorname{dvol}(X, Y, V, W)\right|_{p}$. Thus if we define

$$
\mu_{\mathrm{loc}}(p)(\pi \tilde{X}, \pi \tilde{Y}, \pi \tilde{V}, \pi \tilde{W})=\left.\frac{1}{8 \pi^{2}}|F(p)|^{2} \operatorname{dvol}(X, Y, V, W)\right|_{p},
$$

then (8.7) simplifies to

$$
\begin{aligned}
\mu_{d}(p)(\pi \tilde{X}, \pi \tilde{Y}, \pi \tilde{V}, \pi \tilde{W})= & \mu_{\mathrm{loc}}(p)(\pi \tilde{X}, \pi \tilde{Y}, \pi \tilde{V}, \pi \tilde{W}) \\
& +\left.\operatorname{Rem}_{3}(X, Y, V, W)\right|_{p}+\left.\operatorname{Rem}_{4}(X, Y, V, W)\right|_{p} .
\end{aligned}
$$


Of greatest concern to us will be the local part $\mu_{\text {loc }}(p)$ of this expression. Note that $\mu_{\mathrm{loc}}(p) \wedge \mu_{\mathrm{loc}}(p)=0$, since $d v \mathrm{l}_{p} \wedge d v o l_{p}=0$. However, we will see that $\lim _{q \rightarrow p} \int_{Z} \mu_{\mathrm{loc}}(p) \wedge \mu_{\mathrm{loc}}(q) \neq 0$. In this integral it turns out that instantons of scale $\approx \operatorname{dist}(p, q)$ give the main contribution to the integral. Thus the pullback of $\mu_{d}(p) \wedge \mu_{d}(p)$ to $Z$ can be thought of loosely as a $\delta$-form concentrated on instantons of scale zero.

To integrate $\mu_{d}(p) \wedge \mu_{d}(q)$ we must still worry about the non-local remainder terms $\operatorname{Rem}_{i}$, as well as the fact that the tangent space $T_{[A]} Z$ is not precisely $\pi_{A} \mathcal{H}_{A}$. We will see later that, as $\lambda_{0} \rightarrow 0$, the contributions to the integral of $\mu_{d}(p) \wedge \mu_{d}(q)$ over $Z=Z_{\lambda_{0}}$ from both of these corrections tend to zero. What we wish to compute now is

$$
\lim _{q \rightarrow p} \int_{Z_{\lambda_{0}}} \mu_{\mathrm{loc}}(p) \wedge \mu_{\mathrm{loc}}(q),
$$

where $p$ and $\lambda_{0}$ are fixed.

For given $p, q$, as we integrate $\mu_{\mathrm{loc}}(p) \wedge \mu_{\mathrm{loc}}(q)$ over $Z$, the center point $p_{A}$ of $[A]$ in $Z$ moves around, affecting the support of the vector fields $X, Y, V, W$ in (8.9). Thus for $\mu_{\mathrm{loc}}(p) \wedge \mu_{\mathrm{loc}}(q)\left(\pi \tilde{X}_{1}, \ldots, \pi \tilde{X}_{8}\right)$ to be nonzero, $p_{A}$ must lie in $B\left(p, 8 n_{0} \lambda^{1 / 2}\right) \bigcap B\left(q, 8 n_{0} \lambda^{1 / 2}\right)$. In particular we can restrict $p_{A}$ to a small normal-coordinate ball $U$ centered at $p$ (which we can take to be one of the $U_{i}$ in $(\mathbf{Z 1})$ ) without affecting $\int_{Z} \mu_{\mathrm{loc}}(p) \wedge \mu_{\mathrm{loc}}(q)$. Since we are interested in the limit as $q \rightarrow p$, we may also assume $q \in U$.

Let $2 L=\operatorname{dist}(p, q)$; we will later send $L$ to zero. Define $Z_{1} \subset Z$ to be the set of instantons in $Z$ obeying the two criteria

$$
\begin{gathered}
L^{0.1} \geq \lambda^{1 / 2} \geq L, \\
p_{A} \in B\left(p, n_{0} \lambda^{1 / 2}\right) .
\end{gathered}
$$

Note that if $[A] \in Z_{1}$ then $p_{A} \in B\left(q,\left(n_{0}+1\right) \lambda^{1 / 2}\right)$, so that the cutoff $\beta$ in the definition of the vector fields $X_{i}$ equals 1 at both $p$ and $q$. We will see later that the contribution to (8.11) from the complement of $Z_{1}$ is negligible.

Let $\left\{x_{\text {old }}^{i}\right\}$ denote normal coordinates on $U$. We change coordinates by setting $x_{\text {new }}=L^{-1} x_{\text {old }}$ and replace the metric $g_{\text {old }}$ on $U$ by $g_{\text {new }}=L^{-2} g_{\text {old }}$. Because of the conformal invariance of $|F|^{2} d v o l, \mu_{\mathrm{loc}}(p) \wedge \mu_{\mathrm{loc}}(q)$ is unaffected by this change. However, since $\lambda=\lambda_{\text {old }}$ represented a distance in the old coordinate system, we now have a rescaled upper cutoff for $\lambda_{\text {new }}=L^{-1} \lambda_{\text {old }}$ on $Z$, namely $\lambda_{0, \text { new }}=\lambda_{0} / L$. Measuring all distances in the new metric, the defining conditions for $Z_{1}$ become

$$
\begin{gathered}
L^{-0.8} \geq \lambda_{\text {new }} \geq L, \\
p_{A} \in B\left(p, N L^{-1 / 2} \lambda_{\text {new }}^{1 / 2}\right) .
\end{gathered}
$$

As $L \rightarrow 0$, several things happen. For $A \in Z,\left|F_{A}\right|$ becomes approximately standard on any fixed ball $B(p, K) ; g_{\text {new }}$ approaches the flat metric $\sum\left(d x_{\text {new }}^{i}\right)^{2}$; and (in the 
rescaled metric and coordinates), $Z_{1}$ becomes an $S O(3)$-bundle over monotonically increasing regions of center-scale space that exhaust $(0, \infty) \times\left(\mathbf{R}^{4}-\{0\}\right)$ as $L \rightarrow 0$. Because of $(\mathbf{Z} \mathbf{1})$, we can identify $Z_{1}$ with ever-increasing subsets $G_{L}$ of $G:=\left(\mathbf{H}^{*} \times\right.$ $\mathbf{H}) / \mathbf{Z}_{2}$. Letting $\mu_{\text {loc }}^{\prime}$ denote the pullback of $\mu_{\text {loc }}$ to $\mathbf{H}^{*} \times \mathbf{H}$, we therefore have

$$
\lim _{L \rightarrow 0} \int_{Z_{1}} \mu_{\mathrm{loc}}(p) \wedge \mu_{\mathrm{loc}}(q)=\frac{1}{2} \lim _{L \rightarrow 0} \int_{G_{L}} \mu_{\mathrm{loc}}^{\prime}(p) \wedge \mu_{\mathrm{loc}}^{\prime}(q),
$$

provided this integral converges.

Let $\bar{\rho}$ be as in $(\mathbf{Z 1} \mathbf{Z} \mathbf{Z} \mathbf{5})$. Write elements of $G$ as pairs $(a, b)$, and write $A_{(a, b)}=$ $\rho(a, b), F_{(a, b)}=F_{A_{(a, b)}}$ as in (Z3). If we define $\mu_{\mathrm{loc}}^{\prime}=\bar{\rho}^{*} \mu_{\mathrm{loc}} \in \Omega^{4}(G)$, then

$$
\int_{G_{L}} \mu_{\mathrm{loc}}^{\prime}(p) \wedge \mu_{\mathrm{loc}}^{\prime}(q)=\int_{G_{L}} \mu_{\mathrm{loc}}^{\prime}(p) \wedge \mu_{\mathrm{loc}}^{\prime}(q)\left(\frac{\partial}{\partial a^{1}}, \ldots, \frac{\partial}{\partial b^{4}}\right) d a^{1} \wedge \ldots \wedge d a^{4} \wedge d b^{1} \wedge \ldots \wedge d b^{4} .
$$

To compute this we need to know $\bar{\rho}_{*(a, b)} \partial / \partial a^{i}, \bar{\rho}_{*(a, b)} \partial / \partial b^{i}$. At each $(a, b)$ define $X_{i}, Y_{i}$ to be the vector fields on $\mathbf{R}^{4}$ induced by $\partial / \partial a^{i}, \partial / \partial b^{i}$ respectively. Temporarily writing $b^{i}=a^{i+4}$ and $Y_{i}=X_{i+4}$, from $(\mathbf{Z} 4)$ there is an $8 \times 8$ matrix $C=I d+$ $\left.O\left(\epsilon_{2}\left(\lambda_{\text {old }}\right)\right)\right)$ for which we have

$$
\bar{\rho}_{*(a, b)} C^{j}{ }_{i} \partial / \partial a^{j}=-\pi_{A_{(a, b)}} \iota_{X_{i}} F_{(a, b)} .
$$

Hence from (8.10), if not for the correction matrix $C$ we would have

$$
\begin{aligned}
\mu_{\mathrm{loc}}^{\prime}(p)\left(\frac{\partial}{\partial a^{1}}, \ldots, \frac{\partial}{\partial a^{4}}\right) & =\mu_{\mathrm{loc}}(p)\left(\pi_{A_{(a, b)}} \tilde{X}_{1}^{A}, \ldots, \pi_{A_{(a, b)}} \tilde{X}_{4}^{A}\right) \\
& =\left(8 \pi^{2}\right)^{-2}\left|F_{A}(p)\right|^{2} \operatorname{dvol}_{p}\left(X_{1}, \ldots, X_{4}\right),
\end{aligned}
$$

with a similar formula if we replace any of the $\partial / \partial a^{i}$ 's by a $\partial / \partial b^{i}$.

Let us ignore, for now, (i) the $O\left(\epsilon_{2}\left(\lambda_{\text {old }}\right)\right)=O\left(\epsilon_{2}\left(L \lambda_{\text {new }}\right)\right)$ difference between the matrix $C$ and the identity, and (ii) the $O\left(\left|x_{\mathrm{old}}\right|^{2}\right)=O\left(L^{2}\left|x_{\text {new }}\right|^{2}\right)$ difference between the true metric on the rescaled ball and the flat metric; we will make the corrections later. Since the Euclidean volume form is $d v o l=d x^{1} \wedge \cdots \wedge d x^{4}$, we will write $d \operatorname{dol}_{p}=d^{4} x_{p}, d v o l_{q}=d^{4} x_{q}$ below. Hence

$$
\mu_{\mathrm{loc}}^{\prime}(p) \wedge \mu_{\mathrm{loc}}^{\prime}(q)\left(\frac{\partial}{\partial a^{1}}, \ldots, \frac{\partial}{\partial b^{4}}\right)=\left(8 \pi^{2}\right)^{-2}\left|F_{A}(p)\right|^{2}\left|F_{A}(q)\right|^{2} d^{4} x_{p} \wedge d^{4} x_{q}\left(X_{1}, \ldots, Y_{4}\right) .
$$

So far we have treated $d^{4} x_{p} \wedge d^{4} x_{q}$ as an 8-form whose arguments are vector fields, but we may as well consider it as an 8-form on the 8-dimensional space $T_{p} M \oplus T_{q} N$. Using the canonical isomorphisms $T_{p} \mathbf{R}^{4} \cong T_{q} \mathbf{R}^{4} \cong \mathbf{R}^{4}$ and our further identification of $\mathbf{R}^{4}$ with $\mathbf{H}$, we can write each $X_{i}, Y_{j}$ in the form $(v, w) \in \mathbf{H} \oplus \mathbf{H}$. In the coordinate system $\left\{x_{\text {new }}^{i}\right\}$, the origin represents $p$, and we may assume that $q$ lies on the 
real axis with coordinate $2 \in \mathbf{H}$. Let $\tau_{1}=1 \in \mathbf{H}$ and let $\left\{\tau_{i}\right\}_{2}^{4}$ be the quaternions $\mathbf{i}, \mathbf{j}, \mathbf{k}$. Then $X_{i}(x)=\tau_{i} a^{-1}(x-b)$ and $Y_{i}(x)=\tau_{i}$. so the corresponding elements in $T_{p} \mathbf{R}^{4} \oplus T_{q} \mathbf{R}^{4} \cong \mathbf{H} \oplus \mathbf{H}$ are $X_{i}^{\prime}=\left(-\tau_{i} a^{-1} b, \tau_{i} a^{-1}(2-b)\right)$ and $Y_{i}^{\prime}=\left(\tau_{i}, \tau_{i}\right)$. Modulo the span of the $Y_{i}^{\prime}$, we have $X_{i}^{\prime}=\left(0,2 \tau_{i} a^{-1}\right):=X_{i}^{\prime \prime}$, so $d^{4} x_{p} \wedge d^{4} x_{q}\left(X_{1}^{\prime}, \ldots, X_{4}^{\prime}, Y_{1}^{\prime}, \ldots Y_{4}^{\prime}\right)=$ $d^{4} x_{p} \wedge d^{4} x_{q}\left(X_{1}^{\prime \prime}, \ldots, X_{4}^{\prime \prime}, Y_{1}^{\prime}, \ldots Y_{4}^{\prime}\right)$. Since $d^{4} x_{p}\left(X_{i}^{\prime \prime}, *, *, *\right)=0$, it follows that $d^{4} x_{p} \wedge$ $d^{4} x_{q}\left(X_{1}^{\prime \prime}, \ldots, X_{4}^{\prime \prime}, Y_{1}^{\prime}, \ldots Y_{4}^{\prime}\right)=d^{4} x_{p}\left(Y_{1}^{\prime}, \ldots, Y_{4}^{\prime}\right) d^{4} x_{q}\left(X_{1}^{\prime \prime}, \ldots, X_{4}^{\prime \prime}\right)$. But $d^{4} x_{p}\left(Y_{1}^{\prime}, \ldots, Y_{4}^{\prime}\right)=$ 1 and $d^{4} x_{q}\left(X_{1}, \ldots, X_{4}\right)=2^{4}|a|^{-4}$. Hence

$$
\left.\mu_{\mathrm{loc}}^{\prime}(p) \wedge \mu_{\mathrm{loc}}^{\prime}(q)\right|_{(a, b)}\left(\frac{\partial}{\partial a^{1}}, \ldots, \frac{\partial}{\partial b^{4}}\right)=\left(8 \pi^{2}\right)^{-2}\left|F_{(a, b)}(0)\right|^{2}\left|F_{(a, b)}(2)\right|^{2} 2^{4}|a|^{-4} .
$$

where $F_{(a, b)}$ is the curvature of the instanton obtained from the action of $(a, b)$ on a reference connection in our fiber. Therefore

$$
\left.\mu_{\mathrm{loc}}^{\prime}(p) \wedge \mu_{\mathrm{loc}}^{\prime}(q)\right|_{(a, b)}=2^{4}\left(8 \pi^{2}\right)^{-2}\left|F_{(a, b)}(0)\right|^{2}\left|F_{(a, b)}(2)\right|^{2}|a|^{-4} d^{4} a \wedge d^{4} b .
$$

Because $\lambda_{0}$ is small, there is a reference connection in our fiber that looks approximately standard on a ball of any fixed large radius, with the approximation getting better as $\lambda_{0} \rightarrow 0$ (the rescaling by $L$ only improves this approximation). Our next approximation is to ignore the difference between the true reference connection $A_{0}$ and the standard instanton; we will deal with the error later. The connections in the limiting $Z_{1}$ are then the orbit of the standard instanton $A_{1}$ under the action of $G$. Hence

$$
\begin{aligned}
\left|F_{(a, b)}\right|^{2}(x) & =\left|\tilde{\Phi}_{(a, b)}^{-1} F_{A_{1}}\right|^{2}(x) \\
& =\left|\frac{d\left(\overline{a^{-1}(x-b)}\right) \wedge d\left(a^{-1}(x-b)\right)}{\left(1+\left|a^{-1}(x-b)\right|^{2}\right)^{2}}\right|^{2} \\
& =\frac{48|a|^{4}}{\left(|a|^{2}+|x-b|^{2}\right)^{4}} .
\end{aligned}
$$

Thus

$$
I_{p}:=\lim _{L \rightarrow 0} \int_{G_{L}} \mu_{\mathrm{loc}}^{\prime}(p) \wedge \mu_{\mathrm{loc}}^{\prime}(q)=36 \pi^{-4} \int_{\mathbf{H}^{*} \times \mathbf{H}} \frac{2^{4}|a|^{4} d^{4} a \wedge d^{4} b}{\left(|a|^{2}+|b|^{2}\right)^{4}\left(|a|^{2}+|2-b|^{2}\right)^{4}},
$$

and provided the error terms we have so far ignored are truly ignorable,

$$
\lim _{L \rightarrow 0} \int_{Z} \mu_{\mathrm{loc}}(p) \wedge \mu_{\mathrm{loc}}(q)=\frac{1}{2} I_{p}
$$

(see 8.16).

Lemma $8.1 I_{p}=1$. 
Proof: First introduce spherical coordinates in $a$-space (with radial variable we call $\lambda$ ) and cylindrical coordinates in $b$-space (with radial variable $r$ ). The integrals over the 3 -sphere in $a$-space and the 2 -sphere in the imaginary subspace of $b$-space are trivial, contributing factors $2 \pi^{2}$ and $4 \pi$ respectively. Thus

$$
I_{p}=36 \pi^{-4} \cdot 8 \pi^{3} \cdot 2^{4} \cdot \int_{\lambda=0}^{\infty} \int_{r=0}^{\infty} \int_{z=-\infty}^{\infty} \frac{\lambda^{7} r^{2}}{\left(\lambda^{2}+r^{2}+z^{2}\right)^{4}\left(\lambda^{2}+r^{2}+(z-2)^{2}\right)^{4}} d z d r d \lambda .
$$

Introducing polar coordinates in the $\lambda-r$ quarter-plane, the angular integration reduces us to an integral over two real variables. Using the Residue Theorem to integrate over $z$ leaves us with a one-dimensional integral that can be computed in closed form, yielding $I_{p}=1$.

In the local calculation we ignored errors from four sources: (i) the contribution from the complement of $Z_{1}$, (ii) the difference between the flat metric and the true metric on the rescaled ball; (iii) the difference between $\bar{\rho}_{*} v$ and $-\pi_{A} \tilde{X}_{v}$ (i.e. the difference between the matrix $C$ and the identity); and (iv) the difference between $|F|_{a, b}$ and the standard instanton of scale $|a|$ and center $b$.

Let us first deal with (i). Since the vector fields $X_{i}$ we feed into $\mu_{\text {loc }}$ are cut off at distances $\geq 2 n_{0} \lambda^{1 / 2}$ from $p_{A}$, the integrand $\mu_{\text {loc }}(p) \wedge \mu_{\text {loc }}(q)\left(X_{1}, \ldots, X_{8}\right)$ vanishes for $p_{A}$ outside the ball $B\left(p, 2 n_{0} \lambda^{1 / 2}\right)$. For purposes of integration we therefore need only that portion of $Z$ lying over a ball of fixed small radius centered at $p$. Because of (6.11), the integrand over such a region is bounded by a constant times the integrand we used in our previous calculation, cut off in certain regions. Since the integrand in (8.24) is integrable over all of $\mathbf{H}^{*} \times \mathbf{H}$, given any exhaustion $W_{1} \subset W_{2} \subset \ldots$ of $\mathbf{H} \times \mathbf{H}$, the integral over the complement of $W_{n}$ goes to zero as $n \rightarrow \infty$. As the sets $G_{L}$ provide such an exhaustion, the integral of $\mu_{\mathrm{loc}}(p) \wedge \mu_{\mathrm{loc}}(q)$ over the complement of $Z_{1}$ tends to zero.

Next we turn to the errors (ii)-(iv) listed above. In place of the set $Z_{1}$ considered in the derivation above, for $K>0$ consider the sets $Z_{K, L}$ defined by $\left\{L^{0.1} \geq\right.$ $\left.\lambda^{1 / 2} \geq L, \quad p_{A} \in B(p, K \lambda)\right\}$. After rescaling by $L$ as before these conditions become $\left\{\lambda_{\text {new }} \geq L, p_{A} \in B\left(p, K \lambda_{\text {new }}\right)\right\}$. This time as $L \rightarrow 0, Z_{K, L}$ exhausts $Z_{K, 0}:=\left\{p_{A} \in\right.$ $\left.B\left(p, K \lambda_{\text {new }}\right)\right\}$, with $\lambda_{\text {new }}$ unrestricted. But convergence of the integral $I_{p}$ implies that given $\epsilon_{3}>0$ we can choose $K$ large enough and $L$ small enough that the integral of the integrand in (8.24) over the complement of $Z_{K, L}$ is less than $\epsilon_{3}$. On the interior set $Z_{K, L}$, hypotheses (Z3-Z4) imply that given $\epsilon_{4}>0$, by taking $L$ sufficiently small we can arrange for the ratio of the true $\mu_{\mathrm{loc}}^{\prime}(p) \wedge \mu_{\mathrm{loc}}^{\prime}(q)$ to be within a multiple $\left(1+\epsilon_{4}\right)^{ \pm 1}$ of the integrand in (8.24) over all of $Z_{K, L}$. (Error (ii) gives an $O\left(\lambda_{\text {old }}\right) \leq O\left(L^{0.2}\right)$ ) contribution to $\epsilon_{4}$; error (iii) a contribution $\epsilon_{2}\left(\lambda_{\text {old }}\right) \leq \epsilon_{2}\left(L^{0.2}\right)$, through the matrix 
C. As for error (iv), in the rescaled metric and coordinates (Z3) implies

$$
\left.|| F_{a, b}\right|_{g_{\text {new }}}-\frac{\sqrt{48}|a|^{2}}{\left(|a|^{2}+|x-b|^{2}\right)^{2}} \mid \leq \epsilon_{1}\left(\lambda_{\text {old }}\right) \lambda_{\text {new }}^{-2},
$$

So that this error gives a contribution $\epsilon_{1}\left(\lambda_{\text {old }}\right) \leq \epsilon_{1}\left(L^{0.2}\right)$ to $\epsilon_{4}$.) Hence we can arrange for the integral of $\int_{Z_{K, L}} \mu_{\mathrm{loc}}^{\prime}(p) \wedge \mu_{\mathrm{loc}}^{\prime}(q)$ over lie within $\epsilon_{3} / 2$ of the integral over $Z_{K, L}$ of the integrand in (8.24). It follows that by choosing $L$ small enough, the errors introduced by our approximations can be made arbitrarily small.

We have now proven the following.

Proposition 8.2 For any $p \in N$,

$$
\lim _{q \rightarrow p} \int_{Z_{\lambda_{0}}} \mu_{\mathrm{loc}}(p) \wedge \mu_{\mathrm{loc}}(q)=\frac{1}{2} .
$$

\section{The nonlocal terms in $\mu_{d}(p) \wedge \mu_{d}(q)$.}

From (8.8-8.10), $\mu_{d}(p) \wedge \mu_{d}(q)$ can be expanded as $\mu_{\mathrm{loc}}(p) \wedge \mu_{\mathrm{loc}}(q)$ plus a remainder. Our next task is to show that, as $\lambda_{0} \rightarrow 0$, the contribution of this remainder to $\int_{Z} \mu_{d}(p) \wedge \mu_{d}(q)$ tends to zero. This will follow from the next proposition, whose proof occupies the remainder of this paper.

Proposition 9.1 Let $\Omega$ be the restriction to $Z_{\lambda_{0}}$ of $\Omega_{\mathcal{M}}:=\mu_{d}(p) \wedge \mu_{d}(q)-\mu_{\mathrm{loc}}(p) \wedge$ $\mu_{\mathrm{loc}}(q) \in \Omega^{8}(\mathcal{M})$. Assuming $(\mathbf{Z} \mathbf{1}-\mathbf{Z} \mathbf{5})$, there exists $\delta>0$ such that

$$
\int_{Z_{\lambda_{0}}} \Omega \leq \mathrm{const} \lambda_{0}^{\delta},
$$

where the constant is independent of $p$ and $q$.

Observe that Propositions 8.2 and 9.1 together prove Theorem 1.2.

Proving Proposition 9.1 requires some bounds on $\operatorname{Rem}_{2}(X, Y)$ for $X, Y \in \mathfrak{h}_{A}$. Before starting to derive these, we need some notational simplification. Below we will be computing many things that are multilinear in data of the form $(\mathbf{b}, a, \alpha) \in$ $T_{p_{A}} N \oplus \mathbf{R} \oplus \Lambda_{+}^{2} T_{p_{A}} N$. Given a single vector field $X$ constructed from such data, we 
can denote the defining data of (6.6) by $\left(\mathbf{b}_{X}, a_{X}, \alpha_{X}\right)$. This notation becomes cumbersome, especially when computing objects that involve more than a single vector field. However, because $\left|X_{(\mathbf{b}, a, \alpha)}\right| \leq c\left(|\mathbf{b}|+(|a|+|\alpha|) \lambda^{-1} r_{A}\right)$, the $a$ and $\alpha$ data always enter our bounds with precisely the same weight, so for shorthand we will generally lump the $a$ and $\alpha$ terms together, and simply call them $a$. Furthermore, for simplicity we will often omit the subscripts $X, Y, \ldots$ in the defining data $\left(\mathbf{b}_{X}, a_{X}, \alpha_{X}\right),\left(\mathbf{b}_{Y}, a_{Y}, \alpha_{Y}\right) \ldots$; the dependence on $X, Y, \ldots$ can be reconstructed from context. E.g. if we write

$$
\mid \text { something bilinear in } X, Y \in \mathfrak{h}_{A} \mid \leq c_{1} b^{2}+c_{2} b a+c_{3} a^{2} \text {, }
$$

then on the RHS the notation has the following meaning:

$$
\begin{aligned}
b^{2} & =\left|\mathbf{b}_{X}\right|\left|\mathbf{b}_{Y}\right| \\
b a & =\left(\left|\mathbf{b}_{X}\right|\left(\left|a_{Y}\right|+\left|\alpha_{Y}\right|\right)+\left|\mathbf{b}_{Y}\right|\left(\left|a_{X}\right|+\left|\alpha_{X}\right|\right)\right), \\
a^{2} & =\left(\left|a_{X}\right|+\left|\alpha_{X}\right|\right)\left(\left|a_{Y}\right|+\left|\alpha_{Y}\right|\right) .
\end{aligned}
$$

If the bilinear quantity is antisymmetric in $X, Y$ (as in Theorem 9.2 below), then the estimate factors through the wedge product, in which case we can take

$$
\begin{aligned}
b^{2} & =\left|\mathbf{b}_{X} \wedge \mathbf{b}_{Y}\right| \\
b a & =\left(\left|\mathbf{b}_{X}\right|\left(\left|a_{Y}\right|+\left|\alpha_{Y}\right|\right)+\left|\mathbf{b}_{Y}\right|\left(\left|a_{X}\right|+\left|\alpha_{X}\right|\right)\right), \\
a^{2} & =\left(\left|a_{X}\right|\left|\alpha_{Y}\right|+\left|\alpha_{X}\right|\left|a_{Y}\right|+\left|\alpha_{X} \wedge \alpha_{Y}\right|\right) .
\end{aligned}
$$

Finally, the notation $x \lesssim y$ means $x \leq c y$ for a constant $c$ that is uniform in all relevant parameters.

With this notation in mind, we have

Proposition 9.2 (a) There exists $\delta>0$ such that

$$
\left\|\operatorname{Rem}_{2}(X, Y)\right\|_{\infty} \lesssim \lambda^{-1+\delta}\left(b^{2}+b a \cdot \lambda^{-1 / 2}+a^{2} \cdot \lambda^{-1 / 2}\right) .
$$

Furthermore, there exists $c_{1}>0$ such that, for $r_{A} \geq c_{1} \lambda^{1 / 2}$, we have the pointwise decay

$$
\left|\operatorname{Rem}_{2}(X, Y)\right| \lesssim r_{A}^{-1}\left(b^{2}+b a \cdot \lambda^{-1 / 2}+a^{2} \cdot \lambda^{-1 / 2}\right)
$$

(b) Let $v, X_{v}, \xi_{v}, \xi_{v}^{\prime}$ be as in hypothesis (Z5) of $\S 7$. If we alter the definition of $\operatorname{Rem}_{2}\left(X_{v}, X_{w}\right)$ by replacing $\xi_{v}$ with $\xi_{v}^{\prime}$, then the bounds above still apply.

We will prove Proposition 9.2 (actually a slightly stronger version) in $\S 10$. Let us assume it for now and move onto its application, the proof of Proposition 9.1. The decay estimate (9.6) is crucial in this proof; the global bound (9.5) does not suffice. 
Proof of Proposition 9.1. By hypothesis (Z1), $\int_{Z} \Omega \leq \sum_{i} \rho_{i}^{*} \Omega$. Since $\Omega \in$ $\Omega^{8}(Z)$ we can write $\Omega=f d v o l L_{Z}^{L^{2}}$, where the function $f$ can be computed at $[A] \in Z$ from any (positively) oriented $L^{2}$-orthonormal basis $\eta_{1}, \ldots, \eta_{8}$ of $T_{[A]} Z$ by

$$
f([A])=\Omega\left(\eta_{1}, \ldots, \eta_{8}\right) .
$$

Similarly, we define $f^{\prime}([A])=\Omega_{\mathcal{M}}\left(\eta_{1}^{\prime}, \ldots, \eta_{8}^{\prime}\right)$, where the $\left\{\eta_{i}^{\prime}\right\}$ are an orthonormal basis for $\pi_{A} \mathcal{H}_{A}$, and set $\Omega^{\prime}=f^{\prime} d v o l_{Z}^{L^{2}} \in \Omega^{8}(Z)$.

We will first show that $\int_{Z} \Omega^{\prime} \leq c \lambda_{0}^{\delta}$ (where $\delta$ is as in Proposition 9.2), and then deduce that the same is true for $\int_{Z} \Omega$.

We proceed to estimate $\Omega^{\prime}$. By Proposition 6.4, an approximately orthonormal basis of $\pi_{A} \mathcal{H}_{A}$, up to a scale factor $\left(8 \pi^{2}\right)^{1 / 2}$, is given by $\left\{\eta_{n}^{\prime}=\pi_{A} \tilde{X}_{\left(\mathbf{b}_{n}, a_{n}, \alpha_{n}\right)}:=\pi \tilde{X}_{n}\right\}_{1}^{8}$ as $\left(\mathbf{b}_{n}, a_{n}, \alpha_{n}\right)$ run over an orthonormal basis of $T_{p_{A}} N \oplus \mathbf{R} \oplus \Lambda_{+}^{2}\left(T_{p_{A}} N\right)$. Applying Gram-Schmidt to $\left\{\pi \tilde{X}_{n}\right\}$, it follows that $f^{\prime} \leq$ const $\cdot \Omega\left(\pi \tilde{X}_{1}, \ldots, \pi \tilde{X}_{8}\right) d v o l_{Z}^{L^{2}}$. Hence from (7.3),

$$
\rho_{i}^{*}\left(\Omega^{\prime}\right) \leq c \Omega\left(\pi \tilde{X}_{1}, \ldots, \pi \tilde{X}_{8}\right) \lambda^{3} d \lambda \wedge d v o l_{S^{3}} \wedge d v o l_{N} .
$$

Symbolically we can write $\Omega^{\prime}=\sum_{i=0}^{3} \Omega_{i}^{\prime}$ as a sum of terms of the form $F^{i} \wedge R e m_{2}^{4-i}$, $0 \leq i \leq 3$. We estimate the integrals of $\Omega_{i}^{\prime}$ one case at a time. Only the the completely nonlocal term $\Omega_{0}^{\prime}$ requires the pointwise decay estimate (9.6); for the remaining terms the uniform bound (9.5) suffices. Bounding $\int \Omega_{3}^{\prime}$ requires some care but we shall see that the integrals of $\Omega_{1}^{\prime}$ and $\Omega_{2}^{\prime}$ can be estimated heavy-handedly.

Case 1: Terms of the form $\operatorname{Rem}_{2}^{4}$.

Let $Z_{2} \subset Z$ denote the subset of connections for which both $\operatorname{dist}\left(p, p_{A}\right)$ and $\operatorname{dist}\left(q, p_{A}\right)$ are $\geq c_{1} \lambda^{1 / 2}$, where $c_{1}$ is as in Proposition 9.2, and let $Z_{1}=Z-Z_{2}$. The sets $Z_{1}, Z_{2}$ are the inverse images of sets $W_{1}, W_{2} \subset\left(0, \lambda_{0}\right) \times N$ under the map sending a connection to its scale and center. If for each $\lambda \in\left(0, \lambda_{0}\right)$ we define $W_{1, \lambda}:=\{y \in N \mid$ $\left.(\lambda, y) \in W_{1}\right\}$, then $W_{1, \lambda}$ is contained in the union of a ball of radius $\lesssim \lambda^{1 / 2}$ centered at $p$ and a similar ball centered at $q$, so $\operatorname{Vol}\left(W_{1, \lambda}\right) \lesssim \lambda^{2}$.

For the orthonormal set $\left\{\left(\mathbf{b}_{n}, a_{n}, \alpha_{n}\right)\right\}$ we may choose four elements of the type $(\mathbf{b}, 0,0)$ and four of the type $(0, *, *)$, all normalized to unit length. Then from (9.6) on $Z_{1}$ we have

$$
\begin{aligned}
\left|\operatorname{Rem}_{2}^{4}\left(X_{1}, \ldots, X_{n}\right)\right| & \lesssim \lambda^{-4+4 \delta} \cdot\left\{\text { coefficient of } b^{4} a^{4} \text { in }\left(b^{2}+b a \lambda^{-1 / 2}+a^{2} \lambda^{-1 / 2}\right)^{4}\right\} \\
& \lesssim \lambda^{-6+4 \delta} .
\end{aligned}
$$

Hence from (9.8)

$$
\int_{\rho_{i}^{-1}\left(Z_{1}\right)} \rho_{i}^{*} \Omega_{0}^{\prime} \lesssim \int_{W_{1}} \lambda^{-6+4 \delta} \lambda^{3} d \lambda d v o l_{N} \lesssim \int_{0}^{\lambda_{0}}\left(\lambda^{-3+4 \delta} \operatorname{vol}\left(W_{1, \lambda}\right)\right) d \lambda \lesssim \lambda_{0}^{4 \delta} ;
$$

the integral over the gluing-parameter space $S^{3}$ gives a constant factor. 
Similarly, on $Z_{2},\left|\operatorname{Rem}_{2}^{4}\left(\tilde{X}_{1}, \ldots, \tilde{X}_{n}\right)\right| \lesssim \lambda^{-2} r_{A}(p)^{-2} r_{A}(q)^{-2}$; the two distances $r_{A}(p), r_{A}(q)$ enter this way because in the $\operatorname{Rem}_{2}^{4}$ term in $\mu(p) \wedge \mu(q)$, two of the $R e m_{2}$ 's are evaluated at $p$ and two at $q$ (see (8.10)). Since $r_{A}(p)^{-2} r_{A}(q)^{-2} \leq r_{A}(p)^{-4}+r_{A}(q)^{-4}$ and on $W_{2}$ both $r_{A}(p)$ and $r_{A}(q)$ are $\geq c \lambda^{1 / 2}$, we have

$$
\int_{\rho_{i}^{-1}\left(Z_{2}\right)} \rho_{i}^{*} \Omega_{0}^{\prime} \lesssim \int_{0}^{\lambda_{0}} \lambda^{3} d \lambda\left(\int_{c \lambda^{1 / 2}}^{\operatorname{diam}(N)} \lambda^{-2} r^{-4} r^{3} d r\right) \lesssim \int_{0}^{\lambda_{0}} \lambda|\log \lambda| d \lambda \lesssim \lambda_{0}^{1.99} .
$$

Combining this with the integral over $Z_{1}$ and summing over $i$,

$$
\int_{Z} \Omega_{0}^{\prime} \lesssim \lambda_{0}^{4 \delta}
$$

Case 2: Terms of the form $F \wedge \operatorname{Rem}_{2}^{3}$.

In this and the remaining cases, $F\left(X_{i}, X_{j}\right)$ is computed either at $p$ or at $q$, and since the $X_{i}$ are cut off outside a ball of radius $\sim \lambda^{1 / 2}$ centered at $p_{A}$, for $i \geq 1$ terms of the form $\left.F^{i} \wedge \operatorname{Rem}_{2}^{4-i}\left(X_{1}, \ldots, X_{8}\right)\right|_{p, q}$ vanish unless $\left(\lambda, p_{A}\right)$ lies in the set $Z_{1}$ defined in Case 1. All points $p_{A}$ in the remaining computations can thus be assumed to lie in a single one of our sets $U_{j}$, and $\int_{\rho_{j}^{-1}(Z)} \rho^{*} \Omega_{i}^{\prime}=\int_{Z} \Omega_{i}^{\prime}$.

Note that all vector fields $X, Y \in \mathfrak{h}_{A}$ satisfy $|X|,|Y| \leq \beta\left(b+a \lambda^{-2} r_{A}\right) \leq b+a \lambda^{-1 / 2}$, and hence $|F(X, Y)| \leq|F|\left(b+a \lambda^{-1 / 2}\right)^{2}$. Using the uniform bound (9.5) to estimate the three $R_{e} m_{2}$ terms, we obtain the pointwise bound

$$
\begin{aligned}
\left|F \wedge \operatorname{Rem}_{2}^{3}\left(X_{1}, \ldots, X_{8}\right)\right| & \lesssim|F| \cdot\left\{\text { coefficient of } b^{4} a^{4}\right. \text { in } \\
& \left.\left(b+a \lambda^{-1 / 2}\right)^{2} \lambda^{-3+3 \delta}\left(b^{2}+b a \lambda^{-1 / 2}+a^{2} \lambda^{-1 / 2}\right)^{3}\right\} \\
& \lesssim|F| \lambda^{-5+3 \delta}
\end{aligned}
$$

where $F$ is evaluated at either $p$ or $q$. Because of the cutoff in $X_{i}$ we may assume that $p_{A}$ is a distance $\lesssim c \lambda^{1 / 2}$ from whichever of these points at which we evaluate. Hence, using (6.11),

$$
\int_{Z} \Omega_{1}^{\prime} \lesssim \int_{0}^{\lambda_{0}} \lambda^{3} d \lambda\left(\int_{0}^{c \lambda^{1 / 2}} \frac{\lambda^{-5+3 \delta} \lambda^{2}}{\left(\lambda^{2}+r^{2}\right)^{2}} r^{3} d r\right) \lesssim \int_{0}^{\lambda_{0}} \lambda^{3 \delta}|\log \lambda| d \lambda \lesssim \lambda_{0}^{1+2 \delta}
$$

Case 3: Terms of the form $F^{2} \wedge \operatorname{Rem}_{2}^{2}$.

Here there are two sub-cases, depending on where the points at which $F$ and $\operatorname{Rem}_{2}$ are evaluated; we can have terms of type $F(p) F(p) \operatorname{Rem}_{2}(q) \operatorname{Rem}_{2}(q)$ or of type $F(p) F(q) \operatorname{Rem}_{2}(p) \operatorname{Rem}_{2}(q)$. In each sub-case we bound the $\operatorname{Rem}_{2}$ terms using (9.5). 
At whichever point $F\left(X_{i}, X_{j}\right)$ is evaluated, we can again assume $r_{A} \lesssim \lambda^{1 / 2}$, so that $\left|X_{i}\right| \lesssim b+a \lambda^{-1 / 2}$. Letting $p^{\prime}, p^{\prime \prime}$ denote either of $p, q$, we then have

$$
\begin{aligned}
\left|F^{2} \wedge \operatorname{Rem}_{2}^{2}\left(X_{1}, \ldots, X_{8}\right)\right| & \lesssim|F|\left(p^{\prime}\right)|F|\left(p^{\prime \prime}\right) \cdot\left\{\text { coefficient of } b^{4} a^{4}\right. \text { in } \\
& \left.\left(b+a \lambda^{-1 / 2}\right)^{4} \lambda^{-2+2 \delta}\left(b^{2}+b a \lambda^{-1 / 2}+a^{2} \lambda^{-1 / 2}\right)^{2}\right\} \\
& \lesssim\left(|F|^{2}\left(p^{\prime}\right)+|F|^{2}\left(p^{\prime \prime}\right)\right) \lambda^{-4+2 \delta} .
\end{aligned}
$$

Hence the integral of the different types of $F^{2} R e m_{2}^{2}$ terms can all be bounded by the integral of $|F|^{2}(p) \lambda^{-4+2 \delta}$ :

$$
\int_{Z} \Omega_{2}^{\prime} \lesssim \int_{0}^{\lambda_{0}} \lambda^{3} d \lambda\left(\int_{0}^{c \lambda^{1 / 2}} \frac{\lambda^{-4+2 \delta} \lambda^{4}}{\left(\lambda^{2}+r^{2}\right)^{4}} r^{3} d r\right) \int_{0}^{\lambda_{0}} \lambda^{-1+2 \delta}|\log \lambda| d \lambda \lesssim \lambda_{0}^{\delta} .
$$

Case 4: Terms of the form $F^{3} \wedge \operatorname{Rem}_{2}$.

In the previous two cases we were rather wasteful in bounding $\left|X_{i}\right|$ pointwise; this time we must be more economical.

Since $p$ and $q$ enter the problem symmetrically it suffices to deal with terms of the form $F(p) F(p) F(q) \operatorname{Rem}_{2}(q)$. Temporarily write $r_{p}=r_{A}(p), r_{q}=r_{A}(q), F_{p}=$ $|F|(p), F_{q}=|F|(q)$. Note that for our term to be nonzero, both $r_{p}$ and $r_{q}$ must be $\leq c \lambda^{1 / 2}$. Using this fact several times we find

$$
\begin{aligned}
\left|F^{3} \wedge \operatorname{Rem}_{2}\left(X_{1}, \ldots, X_{8}\right)\right| \lesssim & F_{p}^{2} F_{q} \cdot \lambda^{-1+\delta} \cdot\left\{\text { coefficient of } b^{4} a^{4}\right. \text { in } \\
& \left.\left(b+a \lambda^{-1} r_{p}\right)^{4}\left(b+a \lambda^{-1} r_{q}\right)^{2}\left(b^{2}+b a \lambda^{-1 / 2}+a^{2} \lambda^{-1 / 2}\right)\right\} \\
& \lesssim \lambda^{-4+\delta}\left(F_{p}^{2} F_{q} r_{p}^{2}+F_{p}^{2} F_{q} r_{q}^{2}\right) \\
& \lesssim \lambda^{-4+\delta}\left(F_{p}^{4} r_{p}^{4}+F_{q}^{2}+\lambda^{2} F_{p}^{3}+\lambda^{-4} F_{q}^{3} r_{q}^{6}\right) .
\end{aligned}
$$

We can now replace $p$ by $q$ and integrate over the region $\left\{\left(\lambda, p_{A}\right) \mid 0<\lambda \leq \lambda_{0}, 0 \leq\right.$ $r_{A}(p) \leq c \lambda^{1 / 2}$ as in cases 2 and 3. For each of the four terms $\lambda^{i}|F|^{j} r^{k}$ in parentheses in (9.17), one finds $\int_{0}^{c \lambda^{1 / 2}} \lambda^{i}\left(\lambda^{2} /\left(\lambda^{2}+r^{2}\right)^{2}\right)^{j} r^{k} r^{3} d r \leq$ const, so

$$
\int_{Z} \Omega_{3}^{\prime} \lesssim \int_{0}^{\lambda_{0}} \lambda^{3} \lambda^{-4+\delta} d \lambda \lesssim \lambda_{0}^{\delta}
$$

Combining the four cases, this proves that $\int_{Z} \Omega^{\prime} \leq c \lambda_{0}^{\delta}$ (assuming Proposition $9.2)$.

Now define $\operatorname{Rem}_{2}^{\text {true }}(X, Y)$ to be the right-hand side of (8.6), but with $\xi_{X}, \xi_{Y}$ replaced by the objects $\xi_{X}^{\prime}, \xi_{Y}^{\prime}$ of $(\mathbf{Z 5})$. The form $\Omega$ is obtained from $\Omega^{\prime}$ by replacing each occurrence of $R e m_{2}$ with $R e m_{2}^{\text {true }}$. Hence $\Omega-\Omega^{\prime}$ can be expressed as a sum of terms of the form $F^{i}\left(\operatorname{Rem}_{2}^{\text {true }}-\operatorname{Rem}_{2}\right)^{j} \operatorname{Rem}_{2}^{k}$ for appropriate $i, j, k$. By part (b) of 
Proposition 9.2, the bounds on $\mid\left(\operatorname{Rem}_{2}^{\text {true }}(X, Y)-\operatorname{Rem}_{2}(X, Y) \mid\right.$ are of precisely the same form as in part (a), so the same argument as above shows that $\int_{Z}\left(\Omega-\Omega^{\prime}\right) \leq c \lambda_{0}^{\delta}$, establishing (9.1).

\section{The proof of Proposition 9.2}

The proof of Proposition 9.2 is long, so we outline the strategy. To obtain (9.5), we need a pointwise bound on $G_{0}^{A}\left(\operatorname{Rem}_{2}^{\prime}(X, Y)\right)$ (see (8.6)). If there were a fourdimensional Sobolev embedding $L_{2}^{2} \hookrightarrow C^{0}$, then modulo extra terms arising from Weitzenböck identities that occur when comparing objects of the form $\left\|\nabla^{A} \nabla^{A} \phi\right\|_{2}$ to objects of the form $\Delta^{A} \phi$, we could get a $C^{0}$ bound on $\operatorname{Rem}_{2}(X, Y)$ from an $L^{2}$ bound on $\operatorname{Rem}_{2}^{\prime}(X, Y)$. (This, in turn, would require some $L^{p}$ and/or pointwise bounds on $\xi$.

Of course there is no embedding $L_{2}^{2} \hookrightarrow C^{0}$, but since the failure is borderline, any stronger Sobolev-type norm should give an embedding into $C^{0}$. The most efficient Sobolev inequality for our purposes is the following one. This inequality is not surprising, but may not be widely known, so we prove it in the appendix (Corollary 11.2).

Lemma 10.1 (Sobolev Embedding Lemma.) Let $E$ be a vector bundle over a compact 4-dimensional manifold $N$. For $p \in N$, let $r_{p}$ denote distance to $p$. Then for any $\delta>0$ there exists a constant $c(\delta)$ such that for all connections $\nabla$ on $E$, all $\phi \in \Gamma(E)$, and all $p \in N$,

$$
|\phi(p)| \leq c(\delta)\left(\|\phi\|_{2}+\left\|r_{p}^{-\delta} \nabla \nabla \phi\right\|_{2}\right)
$$

Hence

$$
\|\phi\|_{\infty} \leq c(\delta) \sup _{p \in N}\left(\|\phi\|_{2}+\left\|r_{p}^{-\delta} \nabla \nabla \phi\right\|_{2}\right)
$$

We will use this lemma to get pointwise bounds on $\phi=G_{0}^{A}\left(\operatorname{Rem}_{2}^{\prime}(X, Y)\right)$. Hence we will need to estimate $\left\|G_{0}^{A} \omega\right\|_{2}$ and $\left\|r_{p}^{-\delta} \nabla^{A} \nabla^{A} G_{0}^{A} \omega\right\|_{2}$ for $\omega=\operatorname{Rem}_{2}^{\prime}(X, Y)$. For general $\omega$, Proposition 10.2 estimates these in terms of weighted $L^{2}$ norms of $\omega$, providing bounds whose only dependence on the connection is explicitly through the center point and scale. (This type of uniformity in the connection is the hard part of all our elliptic estimates. Uniformity is important since to estimate an integral over a family of connections we cannot use any bounds that depend on the connection in an uncontrolled way.) Proposition 10.2 also provides similar estimates of objects $\xi$ of the form appearing in (6.14), which we need for reasons discussed below. 
The pointwise estimates of $G_{0}^{A} \omega$ in terms of weighted $L^{2}$ norms of general $\omega$ will be summarized (and generalized) as part of Proposition 10.2, specifically the first half of (10.16). To apply these general estimates to $\omega=\operatorname{Rem}_{2}^{\prime}(X, Y)$ we still need to bound the weighted $L^{2}$ norms of $\operatorname{Rem}_{2}^{\prime}(X, Y)$. To understand what this entails, write $\operatorname{Rem}_{2}^{\prime}(X, Y)=\operatorname{Rem}_{2, \mathrm{loc}}^{\prime}+\operatorname{Rem}_{2, \text { semiloc }}^{\prime}+\operatorname{Rem}_{2, \text { nonloc }}^{\prime}$, where

$$
\operatorname{Rem}_{2, \mathrm{loc}}^{\prime}=R^{\prime \prime}(X, Y), \quad \operatorname{Rem}_{2, \text { semiloc }}^{\prime}=\left\{\tilde{X}, \xi_{Y}\right\}-\left\{\tilde{Y}, \xi_{X}\right\}, \quad \operatorname{Rem}_{2, \text { nonloc }}^{\prime}=\left\{\xi_{X}, \xi_{Y}\right\}
$$

(see (8.4)). Because of the cutoffs in $X$ and $Y$, the expressions $R e m_{2, \text { loc }}^{\prime}$ and $R e m_{2, \text { semiloc }}^{\prime}$ are supported in $B\left(p_{A}, 2 \epsilon\right)$, but $R e m_{2, \text { nonloc }}^{\prime}$ is not. Thus among the estimates we need are weighted $L^{2}$ bounds on $R^{\prime \prime}(X, Y)$. By Lemma 10.4, below, pointwise we find

$$
\left|R^{\prime \prime}(X, Y)\right| \leq c|\hat{X}||\hat{Y}|\left(\beta+\epsilon^{-2} \chi\right)\left(|F|+r_{A}\left|\nabla^{A} F\right|\right)
$$

(recall that $\hat{X}$ is the object that the cutoff $\beta$ multiplies in the definition of $X$ ). Here $\chi$ is the characteristic function of the annulus $\epsilon \leq r_{A} \leq 2 \epsilon$. Thus to apply the estimate (10.16) of Proposition 10.2 to obtain bounds on $G_{0}^{A}\left(\operatorname{Rem}_{2}^{\prime}(X, Y)\right)$, we need to estimate certain expressions of the form $\left\|\beta r_{p}^{-\delta} r_{A}^{m} F\right\|_{2}$, and similar expressions with $F$ replaced by $\nabla^{A} F$ and/or with $\beta$ replaced by $\chi$. This will be accomplished in Lemma 10.5, where we will list all the purely local estimates we need.

Weighted $L^{2}$-norm bounds on $R e m_{2 \text {,semiloc }}^{\prime}$ and $R e m_{2, \text { nonloc }}^{\prime}$ can be obtained from weighted $L^{4}$-norm bounds on $\tilde{X}$ and $\xi$. The first of these is another purely local estimate. The second will be achieved in Proposition 10.8, where we will use the basic elliptic tools in Proposition 10.2 to turn the problem into a local estimate again.

Till now we have made no mention of the role the point $p$ plays in affecting the weighted norms. If we compute these weighted norms as above and take the supremum over $p \in N$ as in (10.2), we obtain only the sup-norm bound (9.5) for $\operatorname{Rem}_{2}(X, Y)$. To prove Proposition 9.1 we additionally need the pointwise decay bound (9.6). Since the local quantities we bound are supported near the center point $p_{A}$ of $A$, decay is only an issue for the nonlocal quantities, but these are built out of Green operators applied to quantities supported near $p_{A}$. Thus one expects that as the distance between $p$ and $p_{A}$ increases, the bounds on our non-local quantities should decrease. This turns out to be true (at least for $\operatorname{dist}\left(p, p_{A}\right) \geq$ const $\left.\cdot \lambda^{1 / 2}\right)$; we simply have to work harder, establishing some general pointwise bounds in Proposition 10.3. Our basic estimates in Proposition 10.2 are most useful for $p$ close to $p_{A}$; to get the bounds that lead to (9.6), in which $p$ is farther from $p_{A}$, we will apply Proposition 10.3.

To establish (9.6) we again break up $\operatorname{Rem}_{2}^{\prime}(X, Y)$ into its local, semi-local, and non-local pieces as in (10.3). In the cases of $R e m_{2, \text { loc }}^{\prime}$ and $R e m_{2, \text { semiloc }}^{\prime}$, Proposition 10.3 again reduces our work to weighted $L^{p}$ bounds of purely local quantities. For $\operatorname{Rem}_{2 \text {,nonloc }}^{\prime}$, however, Proposition 10.3 leaves us with bounding an expression of the form $\left\|r_{A}^{1+\delta^{\prime}}\left\{\xi_{X}, \xi_{Y}\right\}\right\|_{2}$, and the obvious approach-Hölder's inequality and the weighted $L^{4}$ bounds already obtained - does not give us a strong enough bound for 
an adequate decay rate in (9.6). We will circumvent this by obtaining a pointwise decay estimate for $\xi$, which in turn gives us a satisfactory decay rate for $\operatorname{Rem}_{2}$. (In fact, with the pointwise estimate on $\xi$ in hand, it turns out that the contribution of $R e m_{2, \text { nonloc }}^{\prime}$ to $R e m_{2}$ is much smaller than the bounds we obtain from the other two terms.)

With this discussion behind us, our procedure is clear. First we will fill our elliptic toolbox by proving Propositions 10.2 and 10.3. To apply these we need to compute weighted $L^{p}$ norms of various quantities appearing in $R e m_{2}^{\prime}$, which is our next step. The final step is then a matter of bookkeeping, applying the general elliptic tools to bound $G_{0}^{A}$ of $\operatorname{Rem}_{2, \mathrm{loc}}^{\prime}, \operatorname{Rem}_{2, \text { semiloc }}^{\prime}$, and $R e m_{2, \text { nonloc }}^{\prime}$.

To avoid writing similar hypotheses over and over, and for notational simplicity, for the rest of this section we impose the following

Blanket hypotheses and notation. $A$ always denotes a connection with $[A] \in$ $\mathcal{M}_{k+1, \lambda_{0}}^{\kappa, \nu}$ (see Definition 6.2). Every Proposition, Lemma, etc. has an implicit hypothesis "for $\lambda_{0}$ sufficiently small and for all $[A] \in \mathcal{M}_{k+1, \lambda_{0}}^{\prime} \cap \mathcal{M}^{\kappa, \nu}$ ". Constants $c$ are uniform in $A$ and in all other relevant parameters not explicitly shown (though some would depend on $\kappa$ and $\nu$, if these were not fixed); e.g. $c(\delta)$ depends only on $\delta$. Constants are continually updated, and when a hitherto unnamed $c$ appears, there is an implicit "for some constant $c$ ". The notation " $x \lesssim y$ " means $x \leq c y$. $F$ always denotes the curvature of the conection $A$, and $\nabla=\nabla^{A}$ denotes the full covariant derivative on $\Gamma\left(A d P \otimes \Lambda^{*} T^{*} N\right)$ (the tensor product connection determined by $A$ and the Levi-Civita connection). Given any vector field $X$ on $N$, we write $\tilde{X}=\iota_{X} F$ (thus there is an $A$-dependence we suppress). We write $p_{A}$ for the center point of $A$ and $\lambda$ for $\lambda(A)$. For any $p \in N$, we let $r_{p}$ denote distance to $p$, and write $r_{A}$ for $r_{p_{A}}$. When a point $p$ appears in a hypothesis, the letter $d$ always means $r_{A}(p)=\operatorname{dist}\left(p, p_{A}\right)$. The scale $\epsilon=$ const $\lambda^{1 / 2}$ and cutoff $\beta=\beta_{\text {std }}\left(r_{A} / e\right)$ are always as in (6.8), and $\chi$ denotes the characteristic function of the annulus $\left\{\epsilon \leq r_{A} \leq 2 \epsilon\right\}$ containing the support of $d \beta$. We also define the operators $\mathcal{D}=\mathcal{D}^{A}: \Omega^{1}(A d P) \rightarrow \Omega^{0}(A d P) \oplus \Omega_{+}^{2}(A d P)$ by

$$
\mathcal{D}^{A} \eta=\left(\left(d^{A}\right)^{*} \eta, \sqrt{2} d_{+}^{A} \eta\right)
$$

thus $\operatorname{ker}\left(\mathcal{D}^{A}\right)=H_{A}^{1}$, the harmonic space in the middle of the elliptic complex

$$
0 \rightarrow \Omega^{0}(A d P) \stackrel{d^{A}}{\longrightarrow} \Omega^{1}(A d P) \stackrel{\sqrt{2} d_{+}^{A}}{\longrightarrow} \Omega_{+}^{2}(A d P) \rightarrow 0 .
$$

Define $\Delta_{0}^{A}, \Delta_{1}^{A}, \Delta_{+}^{A}$ to be the Laplacians on zero-forms, 1-forms, and SD two-forms, respectively, constructed from this complex, and let $G_{0}^{A}, G_{+}^{A}$ be the inverses of $\Delta_{0}^{A}, \Delta_{+}^{A}$. Also define $\Delta_{\oplus}^{A}, G_{\oplus}^{A}$ on $\Omega^{0}(A d P) \oplus \Omega_{+}^{2}(A d P)$ by $\Delta_{\oplus}^{A}=\Delta_{0}^{A} \oplus \Delta_{+}^{A}, G_{\oplus}^{A}=G_{0}^{A} \oplus G_{+}^{A}$. Note that

$$
\left(\mathcal{D}^{A}\right)^{*}\left(\phi_{0}, \phi_{+}\right)=d^{A} \phi_{0}+\sqrt{2}\left(d_{+}^{A}\right)^{*} \phi_{+},
$$


so that the quantity $\xi_{X}=\xi_{X}^{A}$ of $(6.14)$ can be written as

$$
\xi_{X}=\left(\mathcal{D}^{A}\right)^{*} G_{\oplus}^{A} \mathcal{D}^{A} \tilde{X}
$$

Finally, observe that

$$
\mathcal{D}^{A}\left(\mathcal{D}^{A}\right)^{*}=\Delta_{\oplus}^{A}, \quad\left(\mathcal{D}^{A}\right)^{*} \mathcal{D}^{A}=\Delta_{1}^{A}
$$

Now we can finally begin proving Proposition 9.2. In the following proposition, what drives the estimates are two facts: (i) $\Delta_{\oplus}^{A}$ is uniformly bounded below, and (ii) in the Weitzenböck identity for $\Delta_{\oplus}^{A}$, only Riemannian curvature terms appear; $F$ does not enter.

Proposition 10.2 For $\delta_{0}>0$ sufficiently small and any $\delta, \delta^{\prime}, \delta^{\prime \prime}$ (possibly zero) of absolute value less than $\delta_{0}$, such that for any $p \in N$ and any $\omega \in \Omega^{0}(A d P) \oplus \Omega_{+}^{2}(A d P)$

$$
\left\|G_{\oplus}^{A} \omega\right\|_{2} \lesssim\left\|r_{p}^{1+\delta^{\prime}} \omega\right\|_{2}
$$

and

$$
\left\|r_{p}^{-\delta} \nabla^{A} \nabla^{A} G_{\oplus}^{A} \omega\right\|_{2} \lesssim\left\|r_{p}^{-\delta} \omega\right\|_{2}+\lambda^{\delta^{\prime}-1}\left\|r_{A}^{1-\delta-\delta^{\prime}} \omega\right\|_{2}
$$

Furthermore, if $\xi=\left(\mathcal{D}^{A}\right)^{*} G_{\oplus}^{A} \omega \in \Omega^{1}(A d P)(c f$. (10.8)), then

$$
\begin{aligned}
\|\xi\|_{2} & \lesssim\left\|r_{A}^{1+\delta} \omega\right\|_{2} \\
\left\|r_{A}^{1-\delta} \xi\right\|_{4}+\left\|r_{A}^{1-\delta} \nabla^{A} \xi\right\|_{2} & \lesssim\left\|r_{A}^{1-\delta} \omega\right\|_{2} \\
\left\|r_{p}^{-1-\delta} \xi\right\|_{2}+\left\|r_{p}^{-\delta} \xi\right\|_{4}+\left\|r_{p}^{-\delta} \nabla^{A} \xi\right\|_{2} & \lesssim\left\|r_{p}^{-\delta} \omega\right\|_{2}+\lambda^{\delta^{\prime}-1}\left\|r_{A}^{1-\delta-\delta^{\prime}} \omega\right\|_{2},
\end{aligned}
$$

and

$\left\|r_{p}^{-\delta} \nabla^{A} \xi\right\|_{4}+\left\|r_{p}^{-\delta} \nabla^{A} \nabla^{A} \xi\right\|_{2} \lesssim\left\|r_{p}^{-\delta}\left(\mathcal{D}^{A}\right)^{*} \omega\right\|_{2}+\lambda^{\delta^{\prime}-1}\left\|r_{A}^{-\delta-\delta^{\prime}} \omega\right\|_{2}+\lambda^{\delta^{\prime}-2}\left\|r_{A}^{1-\delta-\delta^{\prime}} \omega\right\|_{2}$.

As a corollary of Lemma 10.1 and (10.12-10.15), if $\delta_{0}>0$ is sufficiently small and $\left.0<\delta<\delta_{0}\right)$ then

$$
\left|G_{\oplus}^{A} \omega\right|(p) \leq c(\delta) \cdot\{\operatorname{RHS} \text { of }(10.11)\} \quad \text { and }|\xi(p)| \lesssim c(\delta) \cdot\{\operatorname{RHS} \text { of }(10.15)\} \text {. }
$$

We remark that in (10.11), (10.14), and (10.15) it is important that $r_{A}$ appear where it does, rather than $r_{p}$, or we would not get strong enough estimates in our applications. The fact that both $r_{A}$ and $r_{p}$ appear together in Proposition 10.2 complicates its proof.

Proof: A slightly less general set of bounds was derived in Lemma 3.3 of [G2] for $\Delta_{+}^{A}$, the Laplacian on SD two-forms only, but for the reasons mentioned prior to stating 
the proposition, essentially the same proof works here. The only differences are that (i) in [G2] the decay (6.11) was true on all of $N$, not merely in $B\left(p_{A}, 2 \epsilon\right)$, and (ii) [G2] dealt only with the case $p=p_{A}$. Since the cited proof is rather long, we will not repeat the parts that require only minor modifications, and will jump to the points of departure.

To establish (10.10), the proof of Lemma 3.3a in [G2] works verbatim to show that

$$
\left\|r_{p}^{-\delta-1} G_{\oplus}^{A} \omega\right\|_{2}+\left\|r_{p}^{-\delta} G_{\oplus}^{A} \omega\right\|_{4}+\left\|r_{p}^{-\delta} \nabla G_{\oplus}^{A} \omega\right\|_{2} \lesssim\left\|r_{p}^{-\delta+1} \omega\right\|_{2} .
$$

Note that $\delta$ need not be positive here. Since $\left|G_{\oplus}^{A} \omega\right| \lesssim\left|r_{p}^{\delta-1} G_{\oplus}^{A} \omega\right|$, (10.10) follows.

Moving to (10.11), let $\eta \in \Omega^{*}(A d P)$ be a form of arbitrary degree. The procedure in [G2] for proving its Lemma 3.3b,c - squaring, integrating by parts, commuting a covariant derivative past a trace Laplacian $\left(\nabla^{A}\right)^{*} \nabla^{A}=\nabla^{*} \nabla$, and juggling termsleads to

$$
\begin{aligned}
\left\|r_{p}^{-\delta} \nabla \eta\right\|_{4}+\left\|r_{p}^{-\delta} \nabla \nabla \eta\right\|_{2} \leq & c\left(\left\|r_{p}^{-\delta} \nabla^{*} \nabla \eta\right\|_{2}+\left\|r_{p}^{-\delta} \eta\right\|_{2}+\left\|r_{p}^{-\delta} \nabla \eta\right\|_{2}\right. \\
& \left.+\left\|r_{A}^{\delta+\delta^{\prime}} r_{p}^{-\delta} F_{A}\right\|_{4}\left\|r_{A}^{-\delta-\delta^{\prime}} \nabla \eta\right\|_{2}\right)
\end{aligned}
$$

here the smallness of $|\delta|$ has also been used to ensure that the term $|\delta|\left\|r_{p}^{-\delta-1} \nabla \eta\right\|_{2}$ that initially comes up on the right-hand side is $\lesssim|\delta|\left\|r_{p}^{-\delta} \nabla \nabla \eta\right\|_{2}$; see [G2], Lemma 3.2. (In [G2] there was no need to insert $r_{A}^{ \pm\left(\delta+\delta^{\prime}\right)}$.) First consider the case $\eta=G_{\oplus}^{A} \omega$, where $\omega \in \Omega^{0}(A d P) \oplus \Omega_{+}^{2}(A d P)$. The Weitzenböck formula gives $\Delta^{A} \eta=\omega+\mathcal{R}\left(G_{\oplus}^{A} \omega\right)$, where $\mathcal{R}$ is an endomorphism proportional to the Riemann tensor. Moreover we will see in Lemma $10.5 \mathrm{~b}$ below that for $\delta, \delta^{\prime}$ sufficiently small, $\left\|r_{A}^{\delta+\delta^{\prime}} r_{p}^{-\delta} F_{A}\right\|_{4} \lesssim \lambda^{\delta^{\prime}-1}$. Inserting these facts into (10.18), one can continue the argument as in [G2] and arrive at an extended version of (10.11):

$$
\left\|r_{p}^{-\delta-1} \nabla G_{\oplus}^{A} \omega\right\|_{2}+\left\|r_{p}^{-\delta} \nabla G_{\oplus}^{A} \omega\right\|_{4}+\left\|r_{p}^{-\delta} \nabla \nabla G_{\oplus}^{A} \omega\right\|_{2} \lesssim\left\|r_{p}^{-\delta} \omega\right\|_{2}+\lambda^{\delta^{\prime}-1}\left\|r_{A}^{1-\delta-\delta^{\prime}} \omega\right\|_{2} .
$$

As for (10.12), since $\left|\mathcal{D}^{*} \eta\right| \leq c|\nabla \eta|$, the desired estimate follows from (10.17).

By similar manipulations one can also establish

$$
\left\|r_{A}^{-\delta+1} \nabla G_{\oplus}^{A} \omega\right\|_{4}+\left\|r_{A}^{-\delta+1} \nabla \nabla G_{\oplus}^{A} \omega\right\|_{2} \lesssim\left\|r_{A}^{1-\delta} \omega\right\|_{2} .
$$

Since $\mathcal{D}$ is $\nabla^{A}$ followed by a covariantly constant projection, the same bounds hold with $\nabla G_{\oplus}^{A} \omega$ replaced by $\mathcal{D}^{*} G_{\oplus}^{A} \omega=\xi$, yielding (10.13). For the same reason, (10.14) follows from (10.19).

Finally, to establish (10.15), return to (10.18) and use the Weitzenböck formula for 1-forms,

$$
\nabla^{*} \nabla \xi=\Delta_{1}^{A} \xi+\mathcal{F}(\xi)+\mathcal{R}(\xi)
$$


Here $\mathcal{F}$ is an endomorphism proportional to $F$. Since $\Delta_{1}^{A} \xi=\mathcal{D}^{*} \mathcal{D}\left(\mathcal{D}^{*} G_{\oplus}^{A} \omega\right)=\mathcal{D}^{*} \omega$ (see (10.9)), we have

$$
\left\|r^{-\delta} \nabla^{*} \nabla \xi\right\|_{2} \leq c\left(\left\|r^{-\delta} \mathcal{D}^{*} \omega\right\|_{2}+\left\|r^{-\delta} \xi\right\|_{2}+\left\|r_{A}^{\delta+\delta^{\prime}} r^{-\delta} F\right\|_{4}\left\|r_{A}^{-\delta-\delta^{\prime}} \xi\right\|_{4}\right),
$$

Hence

$$
\begin{aligned}
\left\|r^{-\delta} \nabla \xi\right\|_{4}+\left\|r^{-\delta} \nabla \nabla \xi\right\|_{2} \leq & c\left(\left\|r^{-\delta}\left(\mathcal{D}^{A}\right)^{*} \omega\right\|_{2}+\left\|r^{-\delta} \xi\right\|_{2}+\left\|r^{-\delta} \nabla \xi\right\|_{2}\right. \\
& \left.+\left\|r_{A}^{\delta+\delta^{\prime}} r^{-\delta} F^{A}\right\|_{4}\left(\left\|r_{A}^{-\delta-\delta^{\prime}} \xi\right\|_{4}+\left\|r_{A}^{-\delta-\delta^{\prime}} \nabla \xi\right\|_{2}\right)\right) .
\end{aligned}
$$

Once again $\left\|r_{A}^{\delta+\delta^{\prime}} r^{-\delta} F\right\|_{4} \lesssim \lambda^{\delta^{\prime}-1}$, and (10.19) (with $\nabla G_{\oplus}^{A} \omega$ replaced by $\xi$ ) implies

$$
\left(\left\|r_{A}^{-\delta-\delta^{\prime}} \xi\right\|_{4}+\left\|r_{A}^{-\delta-\delta^{\prime}} \nabla \xi\right\|_{2}\right) \leq c\left(\left\|r_{A}^{-\delta-\delta^{\prime}} \omega\right\|_{2}+\lambda^{-1+\delta^{\prime \prime}}\left\|r_{A}^{-\delta-\delta^{\prime}-\delta^{\prime \prime}} \omega\right\|_{2}\right) .
$$

Using (10.20-10.19) to bound the other terms in (10.23), the bound (10.15) follows.

Proposition 10.2 gives the same bounds for all $p \in N$; to obtain (9.6), we need estimates that show decay as $d=r_{A}(p)$ grows. The following proposition provides these estimates. We separate the estimates into cases (a) and (b) below because for many purposes the only $\omega$ 's for which we need to estimate the quantities in Proposition 10.2 are compactly supported in a $2 \epsilon$-ball around $p_{A}$, and we get sharper estimates in this case. Part (a) will thus be used to bound the terms $G_{0}^{A} R^{\prime \prime}(X, Y)$ and $G_{0}^{A}\left\{\tilde{X}, \xi_{Y}\right\}$ in $\operatorname{Rem}_{2}(X, Y)$; part (b) will be used to bound $G_{0}^{A}\left\{\xi_{X}, \xi_{Y}\right\}$.

Proposition 10.3 Notation as in Proposition 10.2. There exists $\delta_{0}>0$ such that the following are true.

(a) Suppose that for some $\epsilon_{0}$ (not necessarily related to $\epsilon=c \lambda^{1 / 2}$, and allowed to depend on $\omega)$, (i) $\operatorname{supp}(\omega) \subset B\left(p_{A}, \epsilon_{0}\right)$, (ii) $d=r_{A}(p)=\operatorname{dist}\left(p, p_{A}\right) \geq 2 \epsilon_{0}$, and (iii) $\left|F^{A}\right| \leq B$ on the complement of $\operatorname{supp}(\omega)$. Let $\tilde{\beta}$ be a cutoff function of the form $\beta_{\text {std }}\left(4 r_{p} / d\right)$ (so that $\left.\operatorname{supp}(\tilde{\beta}) \subset B(p, d / 2)\right)$. Then for any $\delta^{\prime}$ with $\left|\delta^{\prime}\right| \leq \delta_{0}$, and any $\delta \in\left(0, \delta_{0}\right)$,

$$
\left|G_{\oplus}^{A} \omega\right|(p) \leq c(\delta) d^{-1-\delta-\delta^{\prime}}\left(1+B^{1 / 2}\right)\left\|r_{A}^{1+\delta^{\prime}} \omega\right\|_{2}
$$

and

$$
|\xi|(p) \leq c(\delta) d^{-\delta-\delta^{\prime}}\left(d^{-2}+B\right)\left\|r_{A}^{1+\delta^{\prime}} \omega\right\|_{2}
$$

Thus, if $\operatorname{supp}(\omega) \subset B\left(p_{A}, 2 \epsilon\right)$ and $d \geq 4 \epsilon=c \lambda^{1 / 2}$, then using (6.10),

$$
\left|G_{\oplus}^{A} \omega\right|(p) \leq c(\delta) d^{-1-\delta-\delta^{\prime}}\left\|r_{A}^{1+\delta^{\prime}} \omega\right\|_{2}
$$


and

$$
|\xi|(p) \leq c(\delta) d^{-2-\delta-\delta^{\prime}}\left\|r_{A}^{1+\delta^{\prime}} \omega\right\|_{2}
$$

(b) Suppose only that $\left|F^{A}\right| \leq B$ on $B(p, d / 2)$, where $d=\operatorname{dist}\left(p, p_{A}\right)>0$; suppose nothing about the support of $\omega$. Let $\tilde{\beta}$ be as in (a). Then for all $\delta^{\prime}$ with $\left|\delta^{\prime}\right| \leq \delta_{0}$, we have

$$
\left|G_{\oplus}^{A} \omega\right|(p) \leq c(\delta)\left(1+B^{1 / 2}\right)\left(d^{-1-\delta-\delta^{\prime}}\left\|r_{A}^{1+\delta^{\prime}} \omega\right\|_{2}+\left\|r_{p}^{-\delta} \tilde{\beta} \omega\right\|_{2}\right)
$$

Thus, if $d \geq c \lambda^{1 / 2}$, then

$$
\left|G_{\oplus}^{A} \omega\right|(p) \leq c(\delta)\left(d^{-1-\delta-\delta^{\prime}}\left\|r_{A}^{1+\delta^{\prime}} \omega\right\|_{2}+\left\|r_{p}^{-\delta} \tilde{\beta} \omega\right\|_{2}\right) .
$$

Proof: (a) We will apply the Sobolev inequality (10.1), but first we must bound $\left\|r_{p}^{-\delta} \nabla \nabla\left(\tilde{\beta} G_{\oplus}^{A} \omega\right)\right\|_{2},\left\|\tilde{\beta} G_{\oplus}^{A} \omega\right\|_{2}$, and similar expressions with $G_{\oplus}^{A} \omega$ replaced by $\xi$.

(i) First we will show that

$$
\left\|r_{p}^{-\delta} \nabla \nabla\left(\tilde{\beta} G_{\oplus}^{A} \omega\right)\right\|_{2} \lesssim d^{-1-\delta-\delta^{\prime}}\left(1+B^{1 / 2}\right)\left\|r_{A}^{1+\delta^{\prime}} \omega\right\|_{2}
$$

Let $\eta \in \Omega^{0}(A d P) \oplus \Omega_{+}^{2}(A d P)$. Proceed as in the proof of Proposition 10.11squaring, integrating by parts, etc.- but this time leave the term proportional to $F$ (which arises from commuting $\nabla^{A}$ past a trace-Laplacian) in integrated form. One arrives at

$$
\left\|r_{p}^{-\delta} \nabla \nabla \eta\right\|_{2}^{2} \lesssim\left\|r_{p}^{-\delta} \Delta \eta\right\|_{2}^{2}+\left\|r_{p}^{-\delta} \eta\right\|_{2}^{2}+\left\|r_{p}^{-\delta} \nabla \eta\right\|_{2}^{2} \int r_{p}^{-2 \delta}|F \| \nabla \eta|^{2}
$$

where $\Delta=\left(\nabla^{A}\right)^{*} \nabla^{A}$. Now replace $\eta$ by $\tilde{\beta} \eta$. In the integral we have $|F| \leq B$, so

$$
\left\|r_{p}^{-\delta} \nabla \nabla(\tilde{\beta} \eta)\right\|_{2}^{2} \leq c\left(\left\|r_{p}^{-\delta} \Delta(\tilde{\beta} \eta)\right\|_{2}^{2}+\left\|r_{p}^{-\delta} \tilde{\beta} \eta\right\|_{2}^{2}+(1+B)\left\|r_{p}^{-\delta} \nabla(\tilde{\beta} \eta)\right\|_{2}^{2}\right) .
$$

An integration by parts, plus various steps already seen in the proof of Lemma 10.2, gives

$$
\left\|r_{p}^{-\delta} \nabla(\tilde{\beta} \eta)\right\|_{2}^{2} \lesssim\left\|r_{p}^{-\delta} \Delta(\tilde{\beta} \eta)\right\|_{2}\left\|r_{p}^{-\delta} \tilde{\beta} \eta\right\|_{2} \leq c\left(k\left\|r_{p}^{-\delta} \Delta(\tilde{\beta} \eta)\right\|_{2}^{2}+k^{-1}\left\|r_{p}^{-\delta} \tilde{\beta} \eta\right\|_{2}^{2}\right)
$$

for arbitrary $k$. Inserting this into $(10.33)$, with $k \lesssim(1+B)^{-1}$, we find

$$
\left\|r_{p}^{-\delta} \nabla \nabla(\tilde{\beta} \eta)\right\|_{2}^{2} \lesssim\left\|r_{p}^{-\delta} \Delta(\tilde{\beta} \eta)\right\|_{2}^{2}+(1+B)\left\|r_{p}^{-\delta} \tilde{\beta} \eta\right\|_{2}^{2} .
$$


Using the Weitzenböck formula as in the proof of Lemma 10.2, we can replace $\Delta$ by $\Delta_{\oplus}^{A}$, absorbing the zeroeth-order term into $(1+B)\left\|r_{p}^{-\delta} \tilde{\beta} \eta\right\|_{2}^{2}$. Additionally, by (10.17) we have $\left\|r_{p}^{-\delta} \tilde{\beta} \eta\right\|_{2} \lesssim\left\|r_{p}^{-\delta} \Delta_{\oplus}^{A}(\tilde{\beta} \eta)\right\|_{2}$. Hence

$$
\left\|r_{p}^{-\delta} \nabla \nabla(\tilde{\beta} \eta)\right\|_{2} \lesssim\left(1+B^{1 / 2}\right)\left\|r_{p}^{-\delta} \Delta_{\oplus}^{A}(\tilde{\beta} \eta)\right\|_{2}
$$

Next, note that for any function $f$,

$$
\left|\Delta_{\oplus}^{A}(f \eta)-f \Delta_{\oplus}^{A} \eta\right| \lesssim(|\nabla \nabla f||\eta|+|\nabla f||\nabla \eta|)
$$

Apply this with $f=\tilde{\beta}$ and $\eta=G_{\oplus}^{A} \omega$, noting that by the hypothesis on the support of $\omega$ we have $\tilde{\beta} \Delta_{\oplus}^{A} \eta=\tilde{\beta} \eta \equiv 0$. Since $\left|\nabla^{j} \tilde{\beta}\right| \leq c d^{-j}$, and since on the support of $\nabla \tilde{\beta}$ we have both $d / 2 \leq r \leq d$ and $d / 2 \leq r_{A} \leq 3 d / 2$, we obtain

$$
\begin{aligned}
\left|r_{p}^{-\delta} \Delta_{\oplus}^{A}(\tilde{\beta} \eta)\right| & \leq c r_{p}^{-\delta} \tilde{\chi}\left(d^{-2}|\eta|+d^{-1}|\nabla \eta|\right) \\
& \leq c d^{-1-\delta-\delta^{\prime}} \tilde{\chi}\left(r_{A}^{-1+\delta^{\prime}}|\eta|+r_{A}^{\delta^{\prime}}|\nabla \eta|\right)
\end{aligned}
$$

where $\tilde{\chi}$ denotes the characteristic function of the annulus $d / 4 \leq r \leq d / 2$. Inserting this into (10.36), we have

$$
\begin{aligned}
\left\|r_{p}^{-\delta} \nabla \nabla(\tilde{\beta} \eta)\right\|_{2} & \leq\left(1+B^{1 / 2}\right) c d^{-1-\delta-\delta^{\prime}}\left(\left\|\tilde{\chi} r_{A}^{-1+\delta^{\prime}} \eta\right\|_{2}+\left\|\tilde{\chi} r_{A}^{\delta^{\prime}} \nabla \eta\right\|_{2}\right) \\
& \leq\left(1+B^{1 / 2}\right) c d^{-1-\delta-\delta^{\prime}}\left(\left\|r_{A}^{-1+\delta^{\prime}} \eta\right\|_{2}+\left\|r_{A}^{\delta^{\prime}} \nabla \eta\right\|_{2}\right)
\end{aligned}
$$

Now apply (10.17) to obtain

$$
\left\|r_{p}^{-\delta} \nabla \nabla(\tilde{\beta} \eta)\right\|_{2} \leq\left(1+B^{1 / 2}\right) c d^{-1-\delta-\delta^{\prime}}\left\|r_{A}^{1+\delta^{\prime}} \omega\right\|_{2},
$$

which leads to (10.31).

Moving on to $\left\|\tilde{\beta} G_{\oplus}^{A} \omega\right\|_{2}$, and repeating some of the steps in the proof of (a) with $\delta=0$, we have

$$
\begin{aligned}
\left\|\tilde{\beta} G_{\oplus}^{A} \omega\right\|_{2} \lesssim\left\|\Delta_{\oplus}^{A}\left(\tilde{\beta} G_{\oplus}^{A} \omega\right)\right\|_{2} & \lesssim d^{-1-\delta^{\prime}}\left(\left\|\tilde{\chi} r_{A}^{-1+\delta^{\prime}} G_{\oplus}^{A} \omega\right\|_{2}+\left\|\tilde{\chi} r_{A}^{\delta^{\prime}} \nabla G_{\oplus}^{A} \omega\right\|_{2}\right) \\
& \lesssim d^{-1-\delta^{\prime}}\left\|r_{A}^{1+\delta^{\prime}} \omega\right\|_{2}
\end{aligned}
$$

This is smaller than the bound (10.31), so (10.1) gives (10.25).

The bound (10.26) is derived by methods similar to the preceding and those used in Proposition 10.2. We leave the details to the reader. 
(b) Proceed as in (a); the only change is that no we no longer have $\tilde{\beta} \omega \equiv 0$. The first effect of this change occurs in (10.38), where we have to add $\left|r^{-\alpha} \tilde{\beta} \omega\right|$ to the RHS. The effect of this term is to add $\left(1+B^{1 / 2}\right)\left\|r^{-\alpha} \tilde{\beta} \omega\right\|_{2}$ to the RHS of $(10.39)$ and (10.40), hence to (10.31). There is a similar change in the bound on $\left\|\tilde{\beta} G_{\oplus}^{A} \omega\right\|_{2}$, but its effect is smaller than the preceding one.

To apply Propositions 10.2 and 10.3 to estimate $R e m_{2}$, we need to estimate expresssions of the form $\left\|r_{p}^{m} \omega\right\|_{2}$ for various $m$, where $\omega=R e m_{2, \text { loc }}^{\prime}, R e m_{2, \text { semiloc }}^{\prime}$, or $\operatorname{Rem}_{2, \text { nonloc }}^{\prime}$ (see 10.3). First we deal with the purely local object $\operatorname{Rem}_{2, \text { loc }}^{\prime}(X, Y)=$ $R^{\prime \prime}(X, Y)$. To start, we need a pointwise estimate, given by the next lemma. The conclusion of the lemma is deceptively simple; the way in which the derivatives of $X$ and $Y$ are coupled to each other and to $\nabla F$ in the definition of $R_{e} m_{2}^{\prime \prime}$ is crucial.

Lemma 10.4 For $X, Y \in \mathfrak{h}_{A}$,

$$
\left|R^{\prime \prime}(X, Y)\right| \lesssim|\hat{X}||\hat{Y}|\left(\beta+\epsilon^{-2} \chi\right)\left(|F|+r_{A}|\nabla F|\right)
$$

(Here $\hat{X}, \hat{Y}$ are the un-cutoff versions of $X, Y$; see (6.8).)

We remark that for general vector fields, this lemma would be false.

Proof: Let $\phi=R^{\prime \prime}(X, Y)$. From (8.4) we have $R^{\prime \prime}(X, Y)=\beta^{2} R^{\prime \prime}(\hat{X}, \hat{Y})+$ terms involving the derivative of $\beta$. The latter are easily dealt with, giving the terms proportional to $\chi$ in $(10.42)$. For $R^{\prime \prime}(\hat{X}, \hat{Y})$, the first three terms in (8.4) have norm bounded by $|F|(|\hat{X}||\hat{Y}|+|\Delta \hat{X}||\hat{Y}|+|\hat{X}||\Delta \hat{Y}|)$, and an easy computation shows that for $X \in \mathfrak{h}_{A},|\Delta \hat{X}| \lesssim|X|$. Furthermore, because $F$ is ASD (and hence Yang-Mills as well) and the "rotational" parts of $\hat{X}, \hat{Y}$ are $\mathrm{SD}$, the remaining three terms in $R^{\prime \prime}(\hat{X}, \hat{Y})$ would vanish if the metric on $N$ were Euclidean. When we do the bookkeeping necessary for the $O\left(r_{A}^{2}\right)$ difference between the metric coefficients $g_{i j}$ and $\delta_{i j}$, the we obtain contributions bounded by $|\hat{X}||\hat{Y}|\left(|F|+r_{A}|\nabla F|\right)$.

Thus, bounding $\left|G_{0}^{A} R^{\prime \prime}(X, Y)\right|$ pointwise boils down to estimates of the form in the following lemma.

Lemma 10.5 Let $p \in N$ be arbitrary and let $d=\operatorname{dist}\left(p, p_{A}\right)$. Then we have the following estimates.

(a) Assume $0 \leq \delta<2$ and $n>-2+\delta$. Then

$$
\left\|\beta r_{p}^{-\delta} r_{A}^{n} F\right\|_{2}+\left\|\beta r_{p}^{-\delta} r_{A}^{n+1} F\right\|_{4}+\left\|\beta r_{p}^{-\delta} r_{A}^{n+1} \nabla F\right\|_{2} \lesssim \begin{cases}\lambda^{n-\delta}, & n-\delta<2, \\ \lambda^{2}|\log \lambda|^{1 / 2} & n-\delta=2, \\ \lambda^{1+(n-\delta) / 2}, & n-\delta>2\end{cases}
$$


and for all $n$

$$
\left\|\chi r_{p}^{-\delta} r_{A}^{n} F\right\|_{2}+\left\|\chi r_{p}^{-\delta} r_{A}^{n+1} \nabla F\right\|_{2} \lesssim \lambda^{1-\delta+n / 2} .
$$
have

(b) Let $\epsilon_{0}>\lambda_{0}^{1 / 2}$ be some fixed number. For $0<\delta<1$ and $-1+\delta<n<3$ we

$$
\left\|r_{p}^{-\delta} r_{A}^{n} F\right\|_{4} \lesssim 1+ \begin{cases}\lambda^{n-1-\delta} & \text { if } d \lesssim \epsilon_{0} \\ \lambda^{n-1} & \text { if } \epsilon_{0} \lesssim d\end{cases}
$$

Proof: (a) First consider the case $\delta=0$. From (6.11) one quickly finds

$$
\left\|\beta r_{A}^{n} F\right\|_{2}+\left\|\beta r_{A}^{n+1} F\right\|_{4} \lesssim\left\{\begin{array}{rr}
\lambda^{n} & -2<n<2, \\
\lambda^{2}|\log \lambda|^{1 / 2} & n=2, \\
\lambda^{1+n / 2} & n>2
\end{array}\right.
$$

and for all $n$,

$$
\left\|\chi r_{A}^{n} F\right\|_{2} \lesssim \lambda^{1+n / 2}
$$

As for $\left\|\beta r_{A}^{n+1} \nabla F\right\|$, the same argument as in the proof of [G1, Lemma 3.3b] shows that

$$
\left\|\beta r_{A}^{n+1} \nabla F\right\|_{2}^{2} \lesssim n\left\|\beta r_{A}^{n} F\right\|_{2}^{2}+\left\|\beta r_{A}^{n+1} F\right\|_{2}^{2}+\left\||d \beta| r_{A}^{n+1} F\right\|_{2}^{2}+\int \beta^{2} r_{A}^{2 n+2}|F|^{3} .
$$

Since $|d \beta| \lesssim \epsilon^{-1} \chi$, we have $|d \beta| r_{A}^{n+1} \leq c \epsilon^{n} \chi$. Thus

$$
\left\|\beta r_{A}^{n+1} \nabla F\right\|_{2} \lesssim\left\|\beta r_{A}^{n} F\right\|_{2}+\lambda^{n / 2}\|\chi F\|_{2}+\left(\int \beta^{2} r_{A}^{2 n+2}|F|^{3}\right)^{1 / 2} .
$$

From (6.11) one can deduce

$$
\left(\int \beta^{2} r_{A}^{2 n+2}|F|^{3}\right)^{1 / 2} \lesssim\left\{\begin{array}{rr}
\lambda^{n}, & n<3, \\
\lambda^{3}|\log \lambda|^{1 / 2}, & n=3, \\
\lambda^{3 n / 2-3 / 2}, & n>3 .
\end{array}\right.
$$

Combining this with our previous bounds we find that

$$
\left\|\beta r_{A}^{n+1} \nabla F\right\|_{2} \lesssim \operatorname{RHS} \text { of(10.46) }
$$

To bound $\left\|\chi r_{A}^{n+1} \nabla F\right\|_{2}$, again use the analysis leading to (10.48), but with $\beta$ replaced by a smooth extension of $\chi$ of the form $f\left(r_{A} / \epsilon\right)$ with $f$ supported in $[1 / 2,3]$. (It is simplest first to note that since $r_{A} \leq 2 \epsilon$ on $\left.\operatorname{supp}(\chi),\left\|\chi r_{A}^{n+1} \nabla F\right\|_{2} \lesssim \lambda^{(n+1) / 2}\|\chi \nabla F\|_{2},\right)$ Then analysis similar to the above leads to

$$
\left\|\chi r_{A}^{n+1} \nabla F\right\|_{2} \lesssim \text { RHS of(10.47). }
$$

This completes the case $\delta=0$ and we move on to the general case. 
We first bound $\left\|\beta r_{p}^{-\delta} r_{A}^{n} F\right\|_{2}$; the method for bounding $\left\|\beta r_{p}^{-\delta} r_{A}^{n+1} F\right\|_{4}$ is identical. Break the ball $B\left(p_{A}, 2 \epsilon\right)$ into two pieces: an inner region $B_{\text {in }}=B(p, d / 2) \bigcap B\left(p_{A}, 2 \epsilon\right)$ and an outer region $B_{\text {out }}=B\left(p_{A}, 2 \epsilon\right)-B_{\text {in }}$. On $B_{\text {out }}$, we have $r_{p} \geq d / 2$, and hence $r_{A} / r_{p} \leq\left(r_{p}+d\right) / r_{p} \leq 3$. Thus

$$
r_{p}^{-\delta} r_{A}^{n}|F|=\left(r_{A} / r_{p}\right)^{\delta} r_{A}^{n-\delta}|F| \lesssim r_{A}^{n-\delta}|F|,
$$

implying

$$
\begin{aligned}
\left\|\beta r_{p}^{-\delta} r_{A}^{n} F\right\|_{L^{2}\left(B_{\text {out }}\right)} \lesssim\left\|\beta r_{A}^{n-\delta} F\right\|_{L^{2}\left(B_{\text {out }}\right)} & \lesssim\left\|\beta r_{A}^{n-\delta} F\right\|_{L^{2}\left(B\left(p_{A}, 2 \epsilon\right)\right)} \\
& \lesssim\left\{\begin{array}{rr}
\lambda^{n-\delta}, & -2<n-\delta<2, \\
\lambda^{2}|\log \lambda|^{1 / 2}, & n-\delta=2, \\
\lambda^{1+(n-\delta) / 2}, & n-\delta>2
\end{array} .\right.
\end{aligned}
$$

For the integral over $B_{\text {in }}$, first suppose $n-\delta \leq 2$ and separately consider the cases $d \leq \lambda, d \geq \lambda$. In both cases note that $d / 2 \leq r_{A} \leq 3 d / 2$ in this region. When $d \leq \lambda$, we then have $r_{A} \lesssim \lambda$ and $|F| \lesssim \lambda^{-2}$ on $B_{\text {in }}$, so

$$
\left\|\beta r_{p}^{-\delta} r_{A}^{n} F\right\|_{L^{2}\left(B_{i n}\right)} \lesssim \lambda^{n-2}\left\|r_{p}^{-\delta}\right\|_{L^{2}\left(B_{i n}\right)} \lesssim \lambda^{n-2} d^{2-\delta} \lesssim \lambda^{n-\delta}
$$

On the other hand if $d \geq \lambda$, then since $r_{A} / d$ is bounded above and below on $B_{\text {in }}$, (6.11) implies $r_{A}^{n}|F| \lesssim \lambda^{2} r_{A}^{n-4} \lesssim \lambda^{2} d^{n-4}$. Hence

$$
\left\|\beta r_{p}^{-\delta} r_{A}^{n} F\right\|_{L^{2}\left(B_{i n}\right)} \lesssim \lambda^{2} d^{n-4}\left\|r_{p}^{-\delta}\right\|_{L^{2}\left(B_{i n}\right)} \lesssim \lambda^{2} d^{n-\delta-2} \leq \lambda^{n-\delta}
$$

since $n-\delta \leq 2$. Combining this with the estimate for $B_{\text {out }}$ we obtain the top two lines of (10.43).

If $n-\delta>2$, separately consider the cases $d \leq 4 \epsilon$ and $d \geq 4 \epsilon$. If $d \leq 4 \epsilon$, the procedure for the case $\delta \geq \lambda$ above yields

$$
\left\|\beta r_{p}^{-\delta} r_{A}^{n} F\right\|_{L^{2}\left(B_{i n}\right)} \lesssim \lambda^{n-2}\left\|r_{p}^{-\delta}\right\|_{L^{2}\left(B_{i n}\right)} \lesssim \lambda^{2} d^{n-\delta-2} \lesssim \lambda^{1+(n-\delta) / 2}
$$

the same bound as on $B_{\text {in }}$. If $d \geq 4 \epsilon$ then on the support of $\beta$ we have $r_{p} \geq \epsilon$, so $r_{p}^{-\delta} r_{A}^{n}|F| \lesssim \lambda^{-\delta / 2} r_{A}^{n}|F|$. Thus (10.46) yields the remaining case of (10.43) for the bound on $\left\|r_{p}^{-\delta} r_{A}^{n} F\right\|_{2}$.

The method for bounding $\left\|\beta r_{p}^{-\delta} r_{A}^{n+1} \nabla F\right\|$, is essentially identical to the method for bounding $\left\|\beta r_{p}^{-\delta} r_{A}^{n+1} \nabla F\right\|_{2}$, except that for the estimates over $B_{i n}$, first multiply by a cutoff function of the form $\beta_{\text {std }}\left(2 r_{p} / d\right)$, and then integrate by parts as in (10.48).

To bound $\left\|\chi r_{p}^{-\delta} r_{A}^{n} F\right\|_{2}$, note that on $\operatorname{supp}(\chi)$ we have $|F| \leq$ const and $r_{A} \leq \epsilon$, so

$$
\left\|\chi r_{p}^{-\delta} r_{A}^{n} F\right\| \lesssim \lambda^{n / 2}\left\|\chi r_{p}^{-\delta}\right\|_{2} \lesssim \lambda^{n / 2}\left\|r_{p}^{-\delta}\right\|_{L^{2}(B(p, 2 \epsilon))} \lesssim \lambda^{1+(n-\delta) / 2}
$$


Similarly $\left\|\chi r_{p}^{-\delta} r_{A}^{n} \nabla F\right\| \lesssim \lambda^{n / 2}\left\|\chi r_{p}^{-\delta} \nabla F\right\|_{2}$, and the same procedure as for $\delta=0$ completes the work.

(b) First write

$$
\left\|r_{p}^{1-\delta} r_{A}^{n} F\right\|_{4} \leq\left\|(1-\tilde{\beta}) r_{p}^{1-\delta} r_{A}^{n} F\right\|_{4}+\left\|\tilde{\beta} r_{p}^{1-\delta} r_{A}^{n} F\right\|_{4},
$$

where $\tilde{\beta}$ is a cutoff of scale $\epsilon_{0}$ centered at $p_{A}$. On the support of $1-\tilde{\beta}$ we have $|F| \leq$ const, so the first term on the RHS is bounded by a constant. The second term can be estimated as in the proof of (b).

We are now in a position to bound $\left|G_{0}^{A} R e m_{2, \text { loc }}^{\prime}\right|$ pointwise, but we postpone this until we have collected the estimates needed to bound the semi-local and non-local contributions to $G_{0}^{A} R e m_{2}^{\prime}$. These require bounds on norms of $\xi_{X}=\mathcal{D}^{*} G_{\oplus}^{A} \mathcal{D} \tilde{X}$, which in turn require pointwise bounds on $\mathcal{D} \tilde{X}$.

Lemma 10.6 For any vector field $X$ on $N$, and any $A S D$ connection $A$, we have the pointwise formulas

$$
\left(d^{A}\right)^{*} \iota_{X} F_{A}=\left\langle d_{+} X^{*}, F_{A}\right\rangle
$$

and

$$
d_{+}^{A}\left(\iota_{X} F_{A}\right)=\operatorname{Sym}_{0}^{2}\left(\nabla X^{*}\right) \sharp F_{A},
$$

where $X^{*}$ is the metric dual of $X, d_{+} X^{*}$ is the self-dual part of $d X^{*}, \operatorname{Sym}_{0}^{2}(T)$ denotes the traceless symmetric part of a rank-two tensor field $T \in \Gamma\left(T^{*} N \otimes T^{*} N\right)$, and, in a local orthonormal basis $\theta^{i}$ of the cotangent bundle, $\langle T, F\rangle=\frac{1}{2} T_{i j} F_{i j} \in$ and $T \sharp F=T_{i j} F_{j k} \theta^{i} \wedge \theta^{k}$. Hence

$$
\left|\mathcal{D}^{A}\left(\iota_{X} F_{A}\right)\right| \lesssim\left(\left|d_{+} X^{*}\right|+\left|\operatorname{Sym}_{0}^{2}\left(\nabla X^{*}\right)\right|\right)\left|F_{A}\right|
$$

and

$$
\begin{aligned}
\left|\nabla^{A} \mathcal{D}^{A}\left(\iota_{X} F_{A}\right)\right| \leq & c\left(\left(\left|\nabla\left(d_{+} X^{*}\right)\right|+\left|\nabla\left(\operatorname{Sym}_{0}^{2}\left(\nabla X^{*}\right)\right)\right|\right)\left|F_{A}\right|\right. \\
& \left.+\left(\left|d_{+} X^{*}\right|+\left|\operatorname{Sym}_{0}^{2}\left(\nabla X^{*}\right)\right|\right)\left|F_{A}\right|\right) .
\end{aligned}
$$

Hence if $X \in \mathfrak{h}_{A}$, then

$$
\left|\mathcal{D}^{A} \tilde{X}\right| \lesssim\left(r_{A} \beta+\epsilon^{-1} \chi\right)|\hat{X}|\left|F_{A}\right|
$$

and

$$
\|\left(\mathcal{D}^{A}\right)^{*} \mathcal{D}^{A} \tilde{X}|\leq c| \nabla^{A} \mathcal{D}^{A} \tilde{X}\left|\lesssim\left(\beta+\epsilon^{-2} \chi\right)\right| \hat{X} \mid\left(\left|F_{A}\right|+r_{A}\left|\nabla^{A} F_{A}\right|\right)
$$


Proof: Using the facts that that $d^{*}=-* d *, d^{A} F=0$, and $* F=-F$, we have

$$
\left(d^{A}\right)^{*}\left(\iota_{X} F\right)=-* d^{A}\left(* \iota_{X}(* F)\right)=* d^{A}\left(X^{*} \wedge F\right)=*\left(d X^{*} \wedge F\right)=\left(d X^{*}, F\right) ;
$$

this gives (10.60). Now fix $p \in N$. Calculating in a local orthonormal frame $\left\{e_{i}\right\}$ of $T N$ and dual coframe $\left\{\theta^{i}\right\}$ with $\left.\nabla e_{i}\right|_{p}=0$,

$$
\begin{aligned}
d_{+}^{A}\left(i_{X} F\right) & =p_{+}\left(\sum \theta^{i} \wedge \iota_{\nabla_{i} X} F\right) \\
& =\sum\left(\nabla_{i} X_{j}\right) p_{+}\left(\theta^{i} \wedge \iota_{e_{j}} F\right) \\
& =p_{+}\left(\operatorname{Sym}^{2}(\nabla X) \sharp F\right)
\end{aligned}
$$

by Lemma 2.3 of [G1]. Since for any symmetric 2-tensor $T$, the pure-trace part of $T$ yields a self-dual 2 -form under the operation $\sharp F$, we may replace $\mathrm{Sym}^{2}$ by $\mathrm{Sym}_{0}^{2}$ in (10.67), and by simple representation theory, the $p_{+}$in (10.67) is redundant.

(10.64-10.65) follow from Lemma 10.6 and a pointwise computation of $d_{+} X^{*}$, $\operatorname{Sym}_{0}^{2}\left(\nabla X^{*}\right)$ that we leave to the reader.

Corollary 10.7 For all $X \in \mathfrak{h}_{A}$, and all $p \in N$, the elements $\tilde{X} \in \mathcal{H}_{A}$ satisfy the following integral bounds.

(a) If $-1<m<2$, then

$$
\left\|r_{A}^{m} \tilde{X}\right\|_{4} \leq c(m) \lambda^{m-1}
$$

(b) If $-1<m \leq 0$, or if $d=\operatorname{dist}\left(p, p_{A}\right) \lesssim \epsilon$ and $-1<m<2$, then

$$
\left\|r_{p}^{m} \mathcal{D}^{A} \tilde{X}\right\|_{2} \leq c(m) \lambda^{m / 2}\left(b \cdot \lambda^{1 / 2}+a\right)
$$

(c) For all $\delta \in(0,1)$,

$$
\left\|r_{p}^{-\delta}\left(\mathcal{D}^{A}\right)^{*} \mathcal{D}^{A} \tilde{X}\right\|_{2} \leq c\left\|r_{p}^{-\delta} \nabla^{A} \mathcal{D}^{A} \tilde{X}\right\|_{2} \lesssim \lambda^{-\delta}\left(b+a \cdot \lambda^{-1 / 2}\right)
$$

Proof: Using (10.64-10.65), plus $|X| \leq b+a \lambda^{-1} r_{A}$, most of these bounds follow directly from Lemma 10.5. The exception is (10.69) in the case $m>0$, for which one must also use the triangle inequality $r_{p} \leq r_{A}+d \lesssim r_{A}+\lambda^{m / 2}$.

We are now in a position to derive our final estimates on the norms of $\xi$ needed to bound $\left|G_{0}^{A} R e m_{2, \text { semiloc }}^{\prime}\right|$ and $\left|G_{0}^{A} R e m_{2 \text {,nonloc }}^{\prime}\right|$ pointwise. We also use the opportunity to prove $(6.13)$. 
Proposition 10.8 There exists $\delta_{0}>0$ such that if $0<\delta<\delta_{0}$ and $0 \leq \delta^{\prime}<\delta_{0}$, then for $\xi=\left(\mathcal{D}^{A}\right)^{*} G_{\oplus}^{A} \mathcal{D}^{A} \tilde{X}$ (with $X \in \mathfrak{h}_{A}$ ) we have the following.

(a) If $0<\delta<\delta_{0}$ then

$$
|\xi(p)| \lesssim \lambda^{\delta^{\prime}}\left(b \cdot \lambda^{-1}+a \cdot \lambda^{-3 / 2}\right) .
$$

If furthermore $d=r_{A}(p) \geq 4 \epsilon=c \lambda^{1 / 2}$, then

$$
|\xi(p)| \leq c(\delta) r_{A}(p)^{-2-\delta-\delta^{\prime}} \lambda^{\delta^{\prime}}\left(b \cdot \lambda+a \cdot \lambda^{1 / 2}\right) .
$$

(b)

$$
\|\xi\|_{2} \lesssim \lambda^{\delta^{\prime} / 2}\left(b \cdot \lambda+a \cdot \lambda^{1 / 2}\right)
$$

Since $\xi_{X}=\tilde{X}-\pi_{A} \tilde{X}$, this implies (6.13).

(c) If $0 \leq \delta \leq \delta_{0}$ then

$$
\left\|r_{p}^{-\delta} \xi\right\|_{4} \lesssim \lambda^{\delta^{\prime}}\left(b+a \cdot \lambda^{-1 / 2}\right) .
$$

(d) If $|\delta|<\delta_{0}$ then

$$
\left\|r_{A}^{1+\delta} \xi\right\|_{4} \lesssim \lambda^{\delta / 2}\left(b \cdot \lambda+a \cdot \lambda^{1 / 2}\right)
$$

Proof: (a) We will omit writing the $\delta$-dependence of the constants. From (10.15) given $\delta, \delta_{0}$ as above there exists $\delta^{\prime}>0$ such that

$$
|\xi(p)| \lesssim\left\|r_{p}^{-\delta}\left(d_{+}^{A}\right)^{*} \omega\right\|_{2}+\lambda^{2 \delta^{\prime}-1}\left\|r_{A}^{-\delta-2 \delta^{\prime}} \omega\right\|_{2}+\lambda^{2 \delta^{\prime}+\delta-2}\left\|r_{A}^{1-2 \delta-2 \delta^{\prime}} \omega\right\|_{2},(10
$$

where $\omega=\mathcal{D}^{A} \tilde{X}$. Using Lemma 10.7, we compute

$$
\lambda^{2 \delta^{\prime}-1}\left\|r_{A}^{-\delta-2 \delta^{\prime}} \omega\right\|_{2}+\lambda^{2 \delta^{\prime}+\delta-2}\left\|r_{A}^{1-2 \delta-2 \delta^{\prime}} \omega\right\|_{2} \lesssim \lambda^{\delta^{\prime}}\left(b \cdot \lambda^{-1}+a \cdot \lambda^{-3 / 2}\right) .
$$

The bound on $\left\|r_{p}^{-\delta}\left(d_{+}^{A}\right)^{*} \omega\right\|_{2}$ from Lemma 10.7 is smaller than this, so we obtain (10.71).

For (10.72), apply (10.28) and Lemma 10.7.

(b), (c), and (d). Apply Proposition 10.2 and Corollary 10.7.

We remark that by using the pointwise decay estimate (10.76) one can obtain the weighted $L^{4}$ decay

$$
\left\|r_{p}^{-\delta}\right\|_{4} \lesssim \lambda^{-\delta} r_{A}(p)^{-\delta}\left(b+a \cdot \lambda^{-1 / 2}\right)
$$

for $d \geq c \lambda^{1 / 2}$, but this is of no help to us.

We're now ready to collate all the estimates needed to prove Theorem 9.2. 
Corollary 10.9 (a) There exist $\delta_{0}>0, \delta^{\prime}>0$ such that for $0 \leq \delta<\delta_{0}$, the following are true.

$$
\begin{aligned}
\left\|r_{p}^{-\delta} \operatorname{Rem}_{2, \mathrm{loc}}^{\prime}(X, Y)\right\|_{2} & \lesssim \lambda^{-\delta / 2}\left(b^{2}+b a \cdot \lambda^{-1 / 2}+a^{2} \cdot \lambda^{-1}\right) . \\
\left\|r_{A}^{1 \pm \delta} \operatorname{Rem}_{2, \mathrm{loc}}^{\prime}(X, Y)\right\|_{2} & \lesssim \lambda^{1 / 2 \pm \delta / 2}\left(b^{2}+b a \cdot \lambda^{-1 / 2}+a^{2} \cdot \lambda^{-1}\right) . \\
\left\|r_{p}^{-\delta} \operatorname{Rem}_{2, \text { semiloc }}^{\prime}\right\|_{2} & \lesssim \lambda^{-1+\delta^{\prime}}\left(b^{2}+b a \cdot \lambda^{-1 / 2}+a^{2} \cdot \lambda^{-1 / 2}\right) . \\
\left\|r_{A}^{1 \pm \delta} \operatorname{Rem}_{2, \text { semiloc }}^{\prime}\right\|_{2} & \lesssim \lambda^{\delta^{\prime}}\left(b^{2}+b a \cdot \lambda^{-1 / 2}+a^{2} \cdot \lambda^{-1 / 2}\right) . \\
\left\|r_{p}^{-\delta} \operatorname{Rem}_{2, \text { nonloc }}^{\prime}(X, Y)\right\|_{2} & \lesssim \lambda^{\delta^{\prime}}\left(b^{2}+b a \cdot \lambda^{-1 / 2}+a^{2} \cdot \lambda^{-1}\right) . \\
\left\|r_{A}^{1 \pm \delta} \operatorname{Rem}_{2, \text { nonloc }}^{\prime}(X, Y)\right\|_{2} & \lesssim \lambda^{1+\delta^{\prime}}\left(b^{2}+b a \cdot \lambda^{-1 / 2}+a^{2} \cdot \lambda^{-1}\right)
\end{aligned}
$$

(b) There exists $\delta^{\prime}>0$ such that for all $p \in N$, the following are true.

$$
\begin{aligned}
\left|G_{0}^{A} \operatorname{Rem}_{2, \mathrm{loc}}^{\prime}(X, Y)\right|(p) & \lesssim \lambda^{-1 / 2+\delta^{\prime}}\left(b^{2}+b a \cdot \lambda^{-1 / 2}+a^{2} \cdot \lambda^{-1}\right) . \\
\left|G_{0}^{A} \operatorname{Rem}_{2, \text { semiloc }}^{\prime}(X, Y)\right|(p) & \lesssim \lambda^{-1+\delta^{\prime}}\left(b^{2}+b a \cdot \lambda^{-1 / 2}+a^{2} \cdot \lambda^{-1 / 2}\right) . \\
\left|G_{0}^{A} \operatorname{Rem}_{2, \text { nonloc }}^{\prime}(X, Y)\right|(p) & \lesssim \lambda^{\delta^{\prime}}\left(b^{2}+b a \cdot \lambda^{-1 / 2}+a^{2} \cdot \lambda^{-1}\right) .
\end{aligned}
$$

(c) There exists $\delta_{0}>0, \delta^{\prime}>0$ such that if $0<\delta<\delta_{0}$ and $d \geq 4 \epsilon=c \lambda^{1 / 2}$ the following are true.

$$
\begin{aligned}
\left|G_{0}^{A} \operatorname{Rem}_{2, \mathrm{loc}}^{\prime}(X, Y)\right|(p) & \lesssim d^{-1-\delta-\delta^{\prime}} \lambda^{\delta^{\prime}}\left(b^{2} \cdot \lambda^{1 / 2}+b a+a^{2} \cdot \lambda^{-1 / 2}\right) \\
\left|G_{0}^{A} \operatorname{Rem}_{2, \text { semiloc }}^{\prime}(X, Y)\right|(p) & \lesssim d^{-1-\delta-\delta^{\prime}} \lambda^{\delta^{\prime}}\left(b^{2}+b a \cdot \lambda^{-1 / 2}+a^{2} \cdot \lambda^{-1 / 2}\right) .(10) \\
\left|G_{0}^{A} \operatorname{Rem}_{2, \text { nonloc }}^{\prime}(X, Y)\right|(p) & \lesssim d^{-1-\delta-\delta^{\prime}} \lambda^{\delta^{\prime}}\left(b^{2} \cdot \lambda+b a \cdot \lambda^{1 / 2}+a^{2}\right)
\end{aligned}
$$

Proof: (a) These bounds follow directly from Lemma 10.4, Lemma Corollary 10.7 the $L^{4}$ bounds in Proposition 10.8, and Hölder's inequality.

(b) Use part (a) and Lemma 10.3.

(c) Since $\operatorname{Rem}_{2, \text { loc }}^{\prime}(X, Y)$ and $\operatorname{Rem}_{2 \text {,semiloc }}^{\prime}(X, Y)$ are supported in $B\left(p_{A}, 2 \epsilon\right)$, for these terms we can apply (10.27) and the corresponding bounds in (a). As $\operatorname{Rem}_{2, \text { semiloc }}^{\prime}(X, Y)$ is not locally supported, we appeal instead to (10.30):

$$
\left|G_{0}^{A}\{\xi, \xi\}\right|(p) \lesssim\left(d^{-1-\delta-\delta^{\prime}}\left\|r_{A}^{1+\delta^{\prime}}\{\xi, \xi\}\right\|_{2}+\left\|r_{p}^{-\delta} \tilde{\beta}\{\xi, \xi\}\right\|_{2}\right)
$$

where $\tilde{\beta}$ is a cutoff of scale $d / 2$ as in Lemma 10.3 .

If we estimate $\left\|r_{A}^{1+\delta^{\prime}}\{\xi, \xi\}\right\|_{2}$ using (10.84) we obtain the right-hand side of (10.90). Were we next to estimate $\left\|r_{p}^{-\delta} \tilde{\beta}\{\xi, \xi\}\right\|_{2}$, the resulting bound would be to large to 
be of use. Instead, since $d \leq r_{A} \leq 3 d$ on the support of $\tilde{\beta}$, we can use the pointwise bound (10.72) to find

$$
\begin{aligned}
\left\|r_{p}^{-\delta} \tilde{\beta}\{\xi, \xi\}\right\|_{2} & \lesssim\left\|r_{p}^{-\delta} \tilde{\beta}\right\|_{2}\left(d^{-2-\delta-\delta^{\prime}} \lambda \delta^{\prime}\left(b \cdot \lambda+a \cdot \lambda^{1 / 2}\right)\right)^{2} \\
& \lesssim d^{-2-3 \delta-2 \delta^{\prime}} \lambda^{2 \delta^{\prime}}\left(b^{2} \cdot \lambda^{2}+b a \cdot \lambda^{3 / 2}+a^{2} \cdot \lambda\right) \\
& \lesssim d^{-1-\delta-\delta^{\prime}} \lambda^{3 / 2-\delta^{\prime \prime}}\left(b^{2} \cdot+b a \cdot \lambda^{-1 / 2}+a^{2} \cdot \lambda^{-1}\right)
\end{aligned}
$$

which is much smaller than our bound on $\left\|r_{A}^{1+\delta^{\prime}}\{\xi, \xi\}\right\|_{2}$. Thus (10.90) follows.

Finally, we have the

Proof of Proposition 9.2. (a) Add the bounds (10.85-10.87) to obtain (9.5). If we add the bounds (10.88-10.90) we obtain a stronger bound than (9.6):

$$
\left|\operatorname{Rem}_{2}(X, Y)\right| \lesssim r_{A}^{-1-\delta^{\prime}} \lambda^{\delta^{\prime}}\left(b^{2}+b a \cdot \lambda^{-1 / 2}+a^{2} \cdot \lambda^{-1 / 2}\right)
$$

(b) In the proof of part (a), the only way in which $\xi$ entered was through the $L^{4}$ bounds on $\left\|r_{p}^{-\delta} \xi_{4}\right\|_{4},\left\|r_{A}^{1+\delta^{\prime}} \xi_{4}\right\|_{4}$, and the pointwise decay (10.72). Hence our assertion follows from the hypothesis $(\mathbf{Z 5})$ of section 7 .

\section{Appendix}

The point of the following weighted Sobolev inequality is that on an $m$-dimensional manifold there is no Sobolev embedding $L_{1}^{m} \hookrightarrow L^{\infty}$, but the failure is borderline. Thus by introducing an arbitrarily small weight into the Sobolev norm we are able to obtain an embedding.

Lemma 11.1 Let $E \rightarrow N$ be a Riemannian vector bundle with metric-compatible connection $\nabla$, where $N$ is compact, Riemannian, and $m$-dimensional $(m>1)$. Given $p \in N$ and $R_{2}>R_{1}>0$, let $\Omega\left(p ; R_{1}, R_{2}\right)$ denote the annulus $\left\{R_{1} \leq r_{p} \leq R_{2}\right\}$, where $r_{p}$ is distance to $p$. There exists a constant $c$ such that for any $\delta>0, R_{2}>R_{1}>0$ (but smaller than the injectivity radius), any $p \in N$, and any $\phi \in \Gamma(E)$ we have

$$
|\phi(p)| \leq c \delta^{-(1-1 / m)} R_{2}^{\delta}\left(\frac{1}{R_{2}-R_{1}}\left\|r_{p}^{-\delta} \phi\right\|_{L^{m}\left(\Omega\left(p ; R_{1}, R_{2}\right)\right)}+\left\|r_{p}^{-\delta} \nabla \phi\right\|_{L^{m}\left(B_{R_{2}}(p)\right)}\right) .
$$

Consequently, 


$$
|\phi(p)| \leq \delta^{-(1-1 / m)}\left(\|\phi\|_{L^{m}(N)}+\left\|r_{p}^{-\delta} \nabla \phi\right\|_{L^{m}(N)}\right)
$$

and

$$
\|\phi\|_{L^{\infty}(N)} \leq c \delta^{-(1-1 / m)}\left(\|\phi\|_{L^{m}(N)}+\sup _{p \in N}\left(\left\|r_{p}^{-\delta} \nabla \phi\right\|_{L^{m}(N)}\right)\right) .
$$

Proof: By Kato's inequality, it suffices to prove this for the trivial real line bundle, i.e. for functions on $N$.

First replace $N$ by $\mathbf{R}^{m}$ and consider a compactly supported function $f \in C_{0}^{\infty}(B(0, R))$. Let $\theta \in S^{m-1}$. Then, using polar coordinates on $B(0, R)$, we have

$$
|f(0)|=\left|\int_{0}^{R} \frac{\partial f}{\partial r}(r, \theta) d r\right| \leq \int_{0}^{R}|\nabla f|(r, \theta) d r
$$

implying

$$
\operatorname{Vol}\left(S^{m-1}\right)|f(0)| \leq \int_{S^{m-1}} d \theta\left(\int_{0}^{R}|\nabla f|(r, \theta) d r\right)=\int_{B(0, R)}|\nabla f| \cdot r^{1-m} d v o l .
$$

Applying the same argument on a normal-coordinate ball $B(p, R)$ in $N$ (where $f \in$ $\left.C_{0}^{\infty}(B(p, R))\right)$, using the compactness of $N$ to get uniformity in the constants below, we obtain

$$
\begin{aligned}
|f(p)| \leq c \int_{B(p, R)}|\nabla f| r_{p}^{1-m} d v o l & =c \int_{B(p, R)} r^{-\delta}|\nabla f| r_{p}^{1-m+\delta} d v o l \\
& \leq c\left\|r_{p}^{-\delta} \nabla f\right\|_{L^{m}(B(p, R))}\left\|r_{p}^{1-m+\delta}\right\|_{L^{m /(m-1)}(B(p, R))} \\
& \leq c \delta^{-(1-1 / m)} R^{\delta}\left\|r_{p}^{-\delta} \nabla f\right\|_{L^{m}(B(p, R))} .
\end{aligned}
$$

Now remove the assumption that $f$ is supported inside a normal coordinate ball. Replace $f$ in the preceding argument by $\beta(r) f$, where $\beta$ is a cutoff function identically 1 for $r \leq R_{1}$ and vanishing for $r \geq R_{2}$; thus $|\nabla \beta| \leq c /\left(R_{2}-R_{1}\right)$. We then have

$$
\begin{aligned}
|f(p)| & \leq c \delta^{-(1-1 / m)} R_{2}^{\delta}\left\|r_{p}^{-\delta} \nabla(\beta f)\right\|_{L^{m}} \\
& \leq c \delta^{-(1-1 / m)} R_{2}^{\delta}\left(\left\|r_{p}^{-\delta}(\nabla \beta) f\right\|_{L^{m}}+\left\|\beta r_{p}^{-\delta} \nabla f\right\|_{L^{m}}\right) \\
& \leq c \delta^{-(1-1 / m)} R_{2}^{\delta}\left(\frac{1}{R_{2}-R_{1}}\left\|r_{p}^{-\delta} f\right\|_{L^{m}\left(\Omega\left(R_{1}, R_{2}, p\right)\right)}+\left\|r_{p}^{-\delta} \nabla f\right\|_{L^{m}\left(B_{R_{2}}(p)\right)}\right)
\end{aligned}
$$

yielding (11.1). Taking $R_{2}=2 R_{1}$ to be, say, half the injectivity radius of $N$, we obtain (11.3).

As a corollary, we have 
Corollary 11.2 Let $E, N, \nabla$ be as in Lemma 11.1, and assume $\operatorname{dim}(N)=4$. Then for all $\delta \in(0,1)$, there exist constants $c(\delta)$ such that for all $\phi \in \Gamma(E)$,

$$
|\phi(p)| \leq c(\delta)\left(\|\phi\|_{L^{2}(N)}+\left\|r_{p}^{-\delta} \nabla \nabla \phi\right\|_{L^{2}(N)}\right),
$$

and hence

$$
\|\phi\|_{\infty} \leq c(\delta) \sup _{p \in N}\left(\|\phi\|_{2}+\left\|r_{p}^{-\delta} \nabla \nabla \phi\right\|_{2}\right) .
$$

Proof: Applying Lemma 11.1 with $m=4$, we have

$$
|\phi(p)| \leq c(\delta)\left(\|\phi\|_{4}+\left\|r_{p}^{-\delta} \nabla \phi\right\|_{4}\right)
$$

Using the Sobolev embedding $L_{1}^{2}(N) \hookrightarrow L^{4}(N)$ we then find

$$
|\phi(p)| \leq c(\delta)\left(\|\phi\|_{2}+\left\|r_{p}^{-\delta-1} \nabla \phi\right\|_{2}+\left\|r_{p}^{-\delta} \nabla \nabla \phi\right\|_{2}\right)
$$

But since $\delta<1$, we also have the weighted Sobolev inequality of Lemma 3.1 of [G2]:

$$
\left\|r^{-1-\delta} \psi\right\|_{2} \leq c\left(\left\|r^{-\delta} \psi\right\|_{2}+\left\|r^{-\delta} \nabla \psi\right\|_{2}\right)
$$

(the proof is again a polar-coordinate computation). Using this we can bootstrap (11.11) into the form (11.8) .

\section{References}

[D1] S. K. Donaldson: Connections, Cohomology and the Intersection Forms of Four Manifolds. J. Diff. Geom. 24 (1986), 275-341.

[D2] S. K. Donaldson: Polynomial Invariants for Smooth 4-manifolds. Topology 29 (1990), 257-315.

[D3] S. K. Donaldson: The orientation of Yang-Mills moduli spaces and 4-manifold topology. J. Diff. Geom. 26 (1987), 397-428.

[DK] S. K. Donaldson and P. Kronheimer: The Geometry of Four-Manifolds. Oxford University Press, Oxford (1990).

[G1] D. Groisser: Curvature of Yang-Mills moduli spaces near the boundary, I. Commun. in Anal. and Geom. 1 (1993) 139-216. 
[G2] D. Groisser: Totally geodesic boundaries of Yang-Mills moduli spaces. Houston J. Math., to appear.

[GP1] and T. H. Parker: The geometry of the Yang-Mills moduli space for definite manifolds. J. Diff. Geom. 29 (1989) 499-544.

[GP2] _ _ Sharp decay estimates for Yang-Mills fields. Commun. in Anal. and Geom., to appear.

[GP3] _ _ Differential forms on the Yang-Mills moduli space. Unpublished notes.

[KM] P. Kronheimer and T. Mrowka: Embedded Surfaces and the Structure of Donaldson's Polynomial Invariants, J. Diff. Geom. 41 (1995), 573-734.

[S1] L. Sadun: A simple geometric representative for $\mu$ of a point. Commun. Math. Phys 178 (1996) 107-113.

[S2] _ _ Simple type is not a boundary phenomenon. In Geometry, Topology and Physics: Proceedings of the First Brazil-USA Workshop, Apanasov et al ed., Walter de Gruyter, Berlin (1997).

[T] C.H. Taubes: Metrics, Connections and Gluing Theorems. CBMS Regional Conference Series in Mathematics 89, American Mathematical Society, Providence (1996). 